Universidad Nacional de La Plata

Facultad de Ciencias Exactas

Departamento de Matemática

\title{
Un estudio conjunto de los grafos cordales y dualmente cordales
}

Pablo De Caria

Tesis Doctoral

Directora: Dra. Marisa Gutierrez

Abril de 2012 
Celebrando el mes del trigésimo quinto aniversario del primer encuentro entre mis padres

En memoria de mi tía Sara 


\section{Quiero agradecer...}

A mis progenitores Francisco y Norma. Por ser mis principales apoyos, por estar conmigo durante tantos momentos de estudio y porque, en su condición de padres, nunca dejan de sorprenderme favorablemente día a día.

A mis hermanas Laura y Mariana y a mi sobrina Ariadna, por tantos momentos compartidos y aún por compartir; y por mantener, si bien ya no nos cobija el mismo techo, el espíritu familiar.

A Hernan y a Paula, por la grata compañia que me han brindado, ayudándome a encontrar más alegría y energía para marchar por la vida. Es mi deseo que el destino nos depare muchas aventuras más.

A María, por tantos años de amistad, por mostrarse permanentemente interesada en lo que hago y por tener siempre algún consejo ante las situaciones que lo ameritan.

A Marisa. Por haberme iniciado en el camino de los grafos y haberme dejado transitarlo durante todos estos años con la suficiente libertad para hacerme sentir cómodo, teniendo siempre algo que decir en el momento oportuno. También quiero reconocer su guia en la realización de muchas gestiones, permitiéndome focalizarme en lo que me importa y salvándome de una burocracia que con facilidad me agobia.

A Juraj, por haber aceptado venir para ser jurado y por la buena predisposición demostrada, que no se restringió en absoluto a la evaluación de esta tesis.

A Liliana y a Oscar, mis otros dos jurados, por el compromiso demostrado para que este trabajo salga lo mejor posible.

A Silvia, mi "camerawoman" oficial de los congresos, por ser una muy buena compañera.

Al CONICET, por haberme provisto de condiciones más que buenas para concentrarme en lo que me gusta, fruto de lo cual surgió este trabajo. 


\section{Índice general}

$\begin{array}{ll}\text { Introducción } & 1\end{array}$

Definiciones $\quad 3$

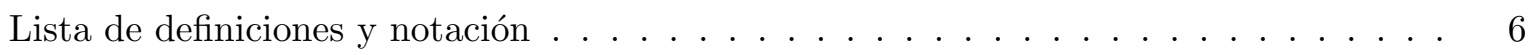

1. Nociones previas $\quad 8$

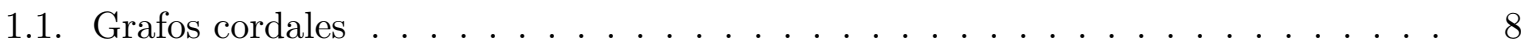

1.1.1. Subclases de los grafos cordales . . . . . . . . . . . . . . . . . 11

1.2. Grafos dualmente cordales . . . . . . . . . . . . . . . . . . . . . . 14

1.2.1. Subclases de los grafos dualmente cordales . . . . . . . . . . . . . . . 16

2. Vértices excéntricos especiales en grafos cordales, dualmente cordales y sus $\begin{array}{ll}\text { subclases } & 18\end{array}$

3. Separadores minimales de vértices de grafos dualmente cordales 26

3.1. Separadores minimales de vértices y árboles compatibles . . . . . . . . . . . . 26

3.2. Separadores minimales de vértices y vecindades . . . . . . . . . . . . 28

3.3. Caracterizaciones . . . . . . . . . . . . . . . . . . . . . . 32

3.4. Separadores minimales de vértices y grafos fuertemente cordales . . . . . . . . 35

4. Grafos básicamente cordales $\quad 38$

4.1. Conjuntos que inducen subárboles y el concepto de base . . . . . . . . . . . . . 41

4.2. Más resultados acerca de los grafos básicamente cordales y dualmente cordales . 45

4.3. Determinando posibles conjuntos de hojas para los árboles compatibles de un grafo dualmente cordal . . . . . . . . . . . . . . . . . . 53

5. Cómo detectar si una familia de árboles es la familia de árboles clique de un grafo cordal

5.1. Un método de detección por conteo . . . . . . . . . . . . . . . 56

5.2. Otro método de detección . . . . . . . . . . . . . . . . . . . 63

5.3. Encontrando todos los grafos cordales con familia de árboles clique dada . . . . . 66

5.4. Detectando familias de árboles compatibles de los grafos dualmente cordales . . . 68

$\begin{array}{ll}\text { 6. Conclusiones } & 71\end{array}$

$\begin{array}{ll}\text { Bibliografía } & 73\end{array}$ 


\section{Introducción}

Los grafos cordales fueron definidos originalmente como aquellos grafos para los cuales todo ciclo de longitud mayor o igual que cuatro posee una cuerda.

Los gafos cordales han sido estudiados exhaustivamente debido a que se les han encontrado muchas aplicaciones, especialmente en el campo de la biología. Como resultado de esas investigaciones, surgieron varias caracterizaciones nuevas de los grafos cordales que involucran diversos conceptos, como los de separador minimal de vértices, vértice simplicial y árbol clique.

Un clique de un grafo $G$ es un conjunto maximal de vértices adyacentes de a pares. El grafo clique de $G$ tiene a los cliques de $G$ como vértices, siendo dos de ellos adyacentes si y sólo si tienen intersección no vacía. Un grafo es dualmente cordal si es el grafo clique de algún grafo cordal.

Históricamente hablando, los grafos dualmente cordales aparecieron hace más de veinte años en varias investigaciones independientes bajo las más diversas denominaciones, como grafos HT, tree clique graphs y árboles expandidos. En cada una de estas investigaciones, los grafos dualmente cordales eran definidos de maneras distintas y fueron necesarios algunos años más hasta que se descubriera que todas las definiciones eran equivalentes. Por esto, podemos afirmar que, al igual que los grafos cordales, los grafos dualmente cordales poseen varias caracterizaciones.

Los resultados que aparecen en este trabajo son numerosos, pero pueden ser clasificados en función de dos objetivos.

En primer lugar, se buscó encontrar nuevas caracterizaciones de los grafos dualmente cordales que resultaran extensiones de las ya conocidas. Esto se ve en el Capítulo 3 y, en menor medida, en el Capítulo 4.

En segundo lugar, dado que varias de las caracterizaciones de los grafos cordales y dualmente cordales son afines, se aprovechan las similaridades para realizar un estudio conjunto de ambas clases en función de esas caracterizaciones. Este es el caso, en mayor o menor medida, de los Capítulos 2, 4 y 5 .

En el Capítulo 1, se repasan las propiedades básicas de los grafos cordales y dualmente cordales.

La clase de los grafos cordales posee varias subclases que serán de nuestro interés, como la de los grafos cordal-potentes, doblemente cordales y fuertemente cordales. Junto con la de los grafos dualmente cordales, todas ellas están caracterizadas por la existencia de vértices especiales: vértices simpliciales para los cordales, vértices con máximo vecino para los dualmente cordales, vértices p-simpliciales en los grafos cordal-potentes, vértices doblemente simpliciales en los doblemente cordales y vértices simples en los fuertemente cordales.

Se sabía que, para un grafo cordal, es cierto que o es completo o posee dos vértices simpliciales no adyacentes. En el Capítulo 2, veremos que eso es también cierto para las otras clases de grafos y sus vértices característicos; y que no resulta complicada la demostración de varias propiedades métricas comunes a todas las clases.

En el Capítulo 3, se obtienen nuevas caracterizaciones de los grafos dualmente cordales 
en términos de sus separadores minimales de vértices. También se aprovecha para realizar un estudio más cabal de los separadores minimales de vértices de los grafos dualmente cordales, siendo estos, entre otras cosas, relacionados con las vecindades de los vértices.

Los grafos cordales y dualmente cordales poseen árboles característicos. Un árbol clique de un grafo cordal es un árbol cuyos vértices son los cliques del grafo $\mathrm{y}$, para todo vértice $v$, el conjunto $\mathcal{C}_{v}$ de cliques que contienen a $v$ induce un subárbol. Un árbol compatible de un grafo dualmente cordal es un árbol generador tal que todo clique induce un subárbol.

Es posible relacionar a los árboles clique con los árboles compatibles a través de los grafos clique. De hecho, todo árbol clique de un grafo cordal es un árbol compatible de su grafo clique. Sin embargo, la recíproca de esta propiedad no es cierta para todos los grafos cordales. Cuando sí es cierta, se dice que el grafo es básicamente cordal. En el Capítulo 4, se buscarán caracterizaciones de los grafos básicamente cordales. Todas las herramientas que se usarán para encontrarlas serán importantes en sí mismas y se verán algunas aplicaciones de ellas. Por ejemplo, se verá cómo varios problemas acerca de los árboles compatibles de un grafo dualmente cordal pueden transformarse en problemas acerca de los árboles clique de un grafo cordal, los cuales previamente han sido más estudiados que los primeros.

Finalmente, en el Capítulo 5, se responderá, dada una familia $\mathcal{T}$ de árboles, si $\mathcal{T}$ es la familia de árboles clique de un grafo cordal y, en caso afirmativo, se caracterizarán los grafos cordales que tienen a $\mathcal{T}$ como familia de árboles clique. También se intentará responder si $\mathcal{T}$ es la familia de árboles compatibles de algún grafo dualmente cordal. 


\section{Definiciones}

Un grafo $G$ es una tripla que consiste de un conjunto de vértices $V(G)$, un conjunto de aristas $E(G)$ y una relación que asocia a cada arista dos vértices (no necesariamente distintos) que reciben el nombre de extremos. $G$ es finito si lo son $V(G)$ y $E(G)$. Es simple si cada arista de $G$ tiene dos extremos distintos, y no hay dos aristas que tengan exactamente los mismos extremos. Todos los grafos que aparecerán en este trabajo son simples y finitos.

Dados $u, v \in V(G), u v$ denota a la arista cuyos extremos son $u$ y $v$. Dos vértices diferentes son adyacentes cuando los dos son los extremos de una misma arista. $G+u v$ es el grafo tal que $V(G+u v)=V(G)$ y $E(G+u v)=E(G) \cup\{u v\} ;$ y $G-u v$ es el grafo tal que $V(G-u v)=V(G)$ y $E(G-u v)=E(G) \backslash\{u v\}$.

El complemento de $G$, o $\bar{G}$, es el grafo con los mismos vértices que $G$ y tal que, para todo par $u, v \in V(G), u v \in E(\bar{G})$ si y sólo si $u \neq v$ y $u v \notin E(G)$.

Un grafo $G^{\prime}$ es un subgrafo de $G$ si $V\left(G^{\prime}\right) \subseteq V(G)$ y $E\left(G^{\prime}\right) \subseteq E(G)$. El subgrafo inducido por $A \subseteq V(G)$, o $G[A]$, tiene a $A$ como conjunto de vértices, siendo dos de ellos adyacentes en $G[A]$ si y sólo si son adyacentes en $G$. El grafo $G-A$ es definido como $G-A=G[V(G) \backslash A]$. Diremos que $G^{\prime}$ es un subgrafo inducido de $G$ si existe $V^{\prime} \subseteq V(G)$ tal que $G^{\prime}=G\left[V^{\prime}\right]$.

$G$ es un grafo completo si todos sus vértices son adyacentes de a pares. Un subconjunto de $V(G)$ es completo si induce un subgrafo completo de $G$. Un clique es un conjunto completo maximal. La familia de cliques de $G$ es simbolizada por $\mathcal{C}(G)$.

Dado $v \in V(G)$, la vecindad abierta de $v$ en $G$, o $N_{G}(v)$, es el conjunto de todos los vértices adyacentes a $v$. La vecindad cerrada de $v$ en $G$, o $N_{G}[v]$, está definida por la igualdad $N_{G}[v]=$ $N_{G}(v) \cup\{v\}$. Es habitual usar la notación $N(v)$ y $N[v]$ cuando es claro sobre qué grafo estamos trabajando. Este comentario también es válido para muchos otros conceptos que se definirán a continuación. El grado de $v$ en $G$, o $\operatorname{deg}_{G}(v)$, es el número de vértices a los cuales $v$ es adyacente en $G$, es decir, $\operatorname{deg}_{G}(v)=\left|N_{G}(v)\right|$. Es $v$ universal si es adyacente a todos los otros vértices de $G$, o sea, $N_{G}[v]=V(G)$. Se dice que $v$ es simplicial si $N_{G}[v]$ es completo. Esto equivale a que $N_{G}[v]$ es un clique. Todo clique que es igual a la vecindad cerrada de algún vértice recibe el nombre de clique simplicial. Si $u$ y $v, u \neq v$, son tales que $N_{G}[u] \subseteq N_{G}[v]$, decimos que $v$ domina a $u$.

Un recorrido de $G$ es una sucesión $v_{1} v_{2} \ldots v_{n}$ de vértices tales que los vértices consecutivos son adyacentes en $G$. La longitud del recorrido es $n-1$. Si todos los vértices del recorrido son diferentes, decimos que es un camino. Si los únicos vértices iguales son el primero y el último, es un ciclo. Una cuerda de un ciclo es una arista cuyos extremos son dos vértices no consecutivos del ciclo.

$G$ es un grafo conexo si, para todo par $u, v \in V(G), G$ tiene un camino que comienza en $u$ y termina en $v$. Un camino así recibe el nombre de uv-camino. Una componente conexa de $G$ es un subgrafo conexo maximal de $G$. Es claro que, si $G$ es conexo, $G$ tiene solamente una componente conexa que es el mismo grafo.

Supongamos que $G$ es conexo, y sean $u$ y $v$ dos vértices no adyacentes de $G$. Un uv-separador es un conjunto $S \subseteq V(G)$ tal que $u$ y $v$ están en diferentes componentes conexas de $G-S$. Diremos 
que el separador es minimal si ningún subconjunto propio de $S$ tiene la misma propiedad.

Los $u v$-separadores minimales poseen propiedades interesantes que valen la pena notar. $\mathrm{Si}$ $S$ es un $u v$-separador minimal, entonces, para todo $s \in S$, existe un $u v$-camino tal que $s$ es su único vértice en $S$ pues, en caso contrario, $S \backslash\{s\}$ también sería un $u v$-separador, lo cual contradice la minimalidad de $S$. El vértice que precede a $s$ en ese camino está en la misma componente conexa de $G-S$ que $u$, y el vértice que lo sucede está en la misma componente conexa de $G-S$ que $v$. Por lo tanto, todo vértice de un $u v$-separador minimal es adyacente a al menos un vértice de la componente conexa de cada vértice que separa. Usaremos simplemente el término separador minimal de vértices para referirnos a un conjunto que separa a algún par de vértices no adyacentes y que es minimal en este sentido. Se denotará por $\mathcal{S}(G)$ a la familia de separadores minimales de vértices de $G$.

La distancia entre $u$ y $v$ en $G$, o $d_{G}(u, v)$, es la longitud de un $u v$-camino en $G$ tal que ningún otro $u v$-camino es más corto. Si no existe ningún $u v$-camino en $G$, entonces $d_{G}(u, v)$ es igual a infinito. El disco centrado en $v$ de radio $k$, o $N_{G}^{k}[v]$, es el conjunto de vértices a distancia a lo sumo $k$ de $v$. La familia de discos de $G$ se denota por $\mathcal{D}(G)$. La $k$-ésima potencia de $G$, o $G^{k}$, es otro grafo con los mismos vértices que $G$, siendo dos de ellos adyacentes en $G^{k}$ si y sólo si la distancia en $G$ entre ellos es a lo sumo $k$. Dicho de otro modo, para todo $v \in V(G)$, $N_{G^{k}}[v]=N_{G}^{k}[v]$.

La excentricidad de $v \in V(G)$ es $\operatorname{ecc}_{G}(v)=\max \left\{d_{G}(v, w), w \in V(G)\right\}$. Diremos que $w$ es un vértice excéntrico de $v$ si ningún vértice de $G$ está más lejos de $v$ que $w$, es decir, $\operatorname{si} \operatorname{ecc}_{G}(v)=$ $d_{G}(v, w)$. Otro concepto métrico importante es el de diámetro. Está definido como la máxima distancia posible entre dos vértices del grafo, o sea, $\operatorname{diam}(G)=\max \left\{d_{G}(v, w): v, w \in V(G)\right\}$.

Dos grafos $G$ y $G^{\prime}$ son isomorfos si existe una función biyectiva $f: V(G) \rightarrow V\left(G^{\prime}\right)$ tal que, para todo par $u, v \in V(G), u v \in E(G)$ si y sólo si $f(u) f(v) \in E\left(G^{\prime}\right)$. A esta función $f$ se la llama isomorfismo. El lector debe tener en cuenta que, en este trabajo, la igualdad y el isomorfismo de grafos tendrán el mismo significado para nosotros.

Un grafo $T$ es un árbol si es conexo y sin ciclos. Un subgrafo conexo de un árbol recibe el nombre de subárbol. Una hoja de un árbol es un vértice de grado uno. $T$ es un árbol generador del grafo $G$ si es un árbol tal que $T$ es un subgrafo de $G$ y $V(T)=V(G)$. Los árboles tienen muchas caracterizaciones. De hecho, son equivalentes:

- $T$ es un árbol.

- $T$ es conexo y $|E(T)|=|V(T)|-1$.

- $T$ no tiene ciclos y $|E(T)|=|V(T)|-1$.

- Para todo par $u, v \in V(T), T$ tiene un único $u v$-camino.

Con respecto al último ítem, llamaremos $T\langle u, v\rangle$ a dicho camino. Se denota por $T[u, v]$ al conjunto de vértices de ese camino y $T(u, v):=T[u, v] \backslash\{u, v\}$. Si $\mathcal{T}$ es una familia de árboles, todos con el mismo conjunto de vértices, y $u$ y $v$ son dos de esos vértices, definamos $\mathcal{T}[u, v]=\bigcup_{T \in \mathcal{T}} T[u, v]$.

La palabra clase es usada para hacer referencia a una familia de grafos. Una clase de grafos es hereditaria si todo subgrafo inducido de un grafo de la clase también está en ésta. Toda familia hereditaria de grafos puede ser caracterizada por la existencia de subgrafos prohibidos minimales, que son grafos que no están en la clase tales que todos sus subgrafos inducidos (diferentes a ellos) sí lo están. Sin embargo, no siempre es fácil determinar cuál es la familia de prohibidos minimales de una clase hereditaria de grafos. 


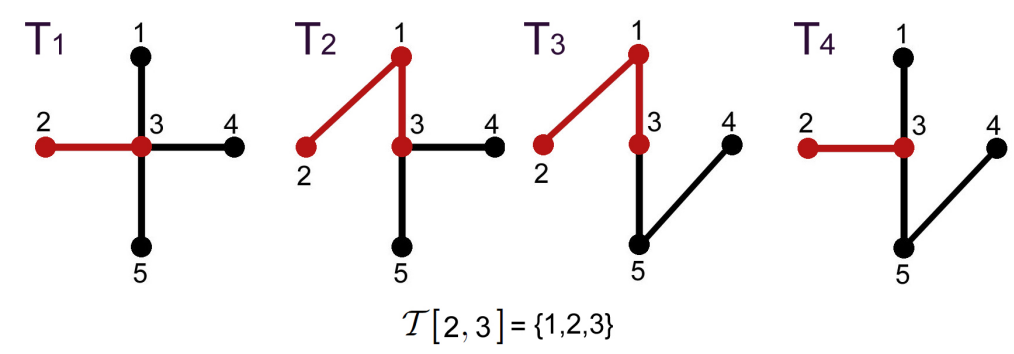

Figura 1: Si se considera la familia de árboles en la figura, $\mathcal{T}[2,3]=\{1,2,3\}$.

Sea $P$ una propiedad de grafos. La clase de los grafos $P$ hereditarios consiste de los grafos $G$ tales que $G$ y todos sus subgrafos inducidos tienen la propiedad $P$. Así definida, esta clase resulta ser claramente hereditaria.

Sea $\mathcal{F}$ una familia de conjuntos no vacíos. Si $F \in \mathcal{F}$, se llama a $F$ miembro de $\mathcal{F}$. Si $v \in \bigcup_{F \in \mathcal{F}} F$, decimos que $v$ es un elemento de la familia. $\mathcal{F}$ es intersectante si la intersección de todo par de miembros de $\mathcal{F}$ es no vacía. $\mathcal{F}$ es Helly si, para toda subfamilia intersectante $\mathcal{F}^{\prime}$ de $\mathcal{F}$, la intersección de todos los miembros de $\mathcal{F}^{\prime}$ es no vacía. Si $\mathcal{C}(G)$ es Helly, diremos que $G$ es un grafo clique-Helly. Llamaremos a $\mathcal{F}$ separadora si, para todo par de elementos distintos $v$ y $w$, existe $F \in \mathcal{F}$ tal que $v \in F$ y $w \notin F$. El grafo de intersección de $\mathcal{F}$, o $L(\mathcal{F})$, tiene a los miembros de $\mathcal{F}$ como vértices, siendo dos de ellos adyacentes si y sólo si no son disjuntos. Nos permitiremos un abuso de terminología para también aplicar el concepto de grafo de intersección a una familia de subárboles de un árbol. En ese caso, consideraremos que dos subárboles se intersecan si poseen al menos un vértice en común. El grafo clique de $G$, o $K(G)$, es el grafo de intersección de $\mathcal{C}(G)$. La función $K$ cuyo dominio y codominio es el conjunto de todos los grafos y que asigna a cada grafo su grafo clique recibe el nombre de operador clique. La dos sección de $\mathcal{F}$, o $S(\mathcal{F})$, es otro grafo cuyos vértices son los elementos de $\mathcal{F}$, siendo dos de ellos adyacentes si y sólo si existe un miembro de $\mathcal{F}$ que los contiene a ambos.

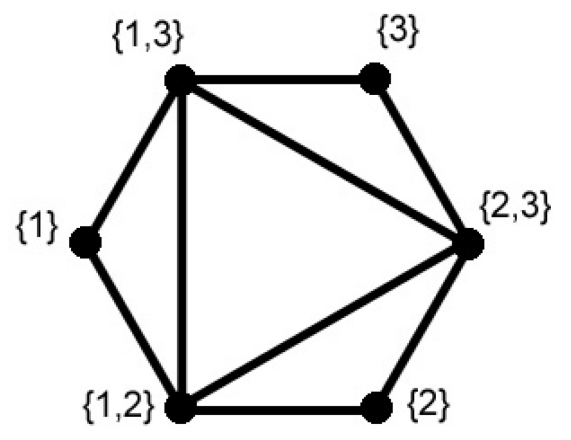

Figura 2: El grafo de intersección de la familia $\{\{1\},\{2\},\{3\},\{1,2\},\{1,3\},\{2,3\}\}$.

La familia dual de $\mathcal{F}$, o $D \mathcal{F}$, está compuesta por los conjuntos $D_{v}=\{F \in \mathcal{F}: v \in F\}$, siendo $v$ un elemento de $\mathcal{F}$. Para el caso particular de la familia $\mathcal{C}(G)$, se usará la notación $\mathcal{C}_{v}$ en lugar de $D_{v}$. También se usará una notación más general: dado un conjunto $A$ de 
vértices, $\mathcal{C}_{A}:=\{C \in \mathcal{C}(G): A \subseteq C\}$.

Dos familias $\mathcal{F}$ y $\mathcal{F}^{\prime}$ son isomorfas si existen dos biyecciones $a: \mathcal{F} \rightarrow \mathcal{F}^{\prime}$ y $b$ : $\bigcup_{F \in \mathcal{F}} F \rightarrow \bigcup_{F \in \mathcal{F}^{\prime}} F$ tales que, para todo $F \in \mathcal{F}, a(F)=b(F)$. La igualdad entre familias y el isomorfismo de familias serán dos cosas equivalentes para nosotros.

\section{Lista de definiciones y notación}

Vértices adyacentes, 3

Árbol, 4

Árbol clique, 10

Árbol generador, 4

Arista, 3

Base, 42

Grafo básicamente cordal, 38

$B_{\mathcal{F}}, 49$

Conjunto booleano positivo, 48

Conjunto booleano positivo conexo, 48

$B_{S}, 50$

$\mathcal{C}(), 3$

$\mathcal{C}_{A}, \mathcal{C}_{S}, 6$

Camino, 3

uv-camino, 3

Camino dirigido, 11

$C h(\mathcal{T}), 67$

Ciclo, 3

Clase de equivalencia, 56

Clase de grafos, 4

Clique, 3

Grafo clique-Helly, 5

Clique simplicial, 3

Árbol compatible, 15

Complemento de un grafo, 3

Conjunto completo, 3

Grafo completo, 3

Componente conexa, 3

Grafo conexo, 3

Conjunto cociente, 56

Grafo cordal, 8

Grafo cordal-potente, 13

Correspondencia, 45

Cuerda, 3

Cuerda fuerte, 13

$\mathcal{C}_{v}, 5$
$\mathcal{D}(), 4$

$d(),, d_{G}(),$,

$\operatorname{deg}(), \operatorname{deg}_{G}(), 3$

$D \mathcal{F}, D \mathcal{C}(G), 5$

$\operatorname{diam}($ ), 4

Diámetro, 4

Digrafo, 11

Dimensión, 44

Dimensión dual, 44

Disco, 4

Distancia, 4

Grafo doblemente cordal, 16

Vértice doblemente simplicial, 16

Vértice dominado, 3

Dos sección, 5

Grafo dualmente cordal, 14

Grafo dualmente $D V, 17$

Grafo dualmente $R D V, 17$

$D_{u v}, 64$

$D_{v}, 5$

Grafo $D V, 11$

$D V$ árbol clique, 12

$D V$ árbol compatible, 17

$D(v, i), 31$

$\operatorname{ecc}(), \operatorname{ecc}_{G}(), 4$

$E(G), 3$

Elemento, 5

Árbol enraizado, 11

$E(\mathcal{T}), 59$

Excentricidad, 4

Vértice excéntrico, 4

Extremos, 3

Familia dual, 5

Grafo finito, 3

Grafo fuertemente cordal, 13

$\bar{G}, 3$ 
$G[], 3$

$G-A, G-v, G-S, 3$

Familia generadora, 42

$G_{i}, 8$

$G^{k}, 4$

Grado, 3

Grafo, 3

Grafo clique, 5

Grafo de intersección, 5

Grafo dirigido, 11

$G-u v, T-u v, 3$

$G+u v, T+u v, 3$

Familia Helly, 5

Clase hereditaria, 4

Grafo $P$ hereditario, 5

Hoja, 4

$H_{\mathcal{T}}, 59$

$H_{\mathcal{T}}^{*}, 59$

$H_{T, S}, 57$

Subgrafo inducido, 3

Familia intersectante, 5

Isomorfismo, 4

Isomorfo, 4, 6

$K(), 5$

$L($ ), 5

$\mathcal{L}(), 53$

Leafage, 54

Leafage dual, 54

Máximo vecino, 14

Miembro, 5

$N\left(\right.$ ),$N_{G}($ ), 3

$N[], N_{G}[], 3$

$N^{k}[], N_{G}^{k}[], 4$

$n K_{2}, 48$

Operador clique, 5

Orden de eliminación perfecto, 8

Orden de eliminación simple, 13

Orden de vecindades máximas, 14

Orden doblemente perfecto, 16

Orden p-perfecto, 13

Par separador, 39

$P\left(H^{\prime}\right), 59$
Potencia de un grafo, 4

Prohibido minimal, 4

Vértice p-simplicial, 13

Raíz, 11

Grafo $R D V, 11$

$R D V$ árbol clique, 12

$R D V$ árbol compatible, 17

Recorrido, 3

Relación de equivalencia, 56

$\mathcal{R}_{H^{\prime}}^{\mathcal{T}}, 61$

$\mathcal{R}_{S}^{G}, 57$

Rueda, 17

$S(), 5$

$\mathcal{S}(), 4$

Conjunto $S$-admisible, 57

$\mathcal{S C}(), 42$

$\mathcal{S D C}(), 42$

uv-separador, 3

Familia separadora, 5

Separador minimal, 4

Grafo simple, 3

Vértice simple, 13

Vértice simplicial, 3

Sol, 12

$S p(\mathcal{F}), 67$

$s(T), 33$

Subárbol, 4

Subgrafo, 3

$\tau($ ), 10

$T(,),$,

$T[],$,

$\mathcal{T}[],$,

$T\langle\rangle,$,

$\mathcal{T}_{G}\left[C_{1}, C_{2}\right], 63$

Unión conexa, 42

Vértice universal, 3

Grafo $U V, 11$

$U V$ árbol clique, 12

$V($ ), 3

Vecindad abierta, 3

Vecindad cerrada, 3

Vértice, 3

$W_{n}, 17$ 


\section{Capítulo 1}

\section{Nociones previas}

En este capítulo, se verán todos los resultados conocidos acerca de los grafos cordales y dualmente cordales que son necesarios para el posterior desarrollo de este trabajo.

\subsection{Grafos cordales}

Se definen a los grafos cordales como aquellos para los cuales todo ciclo de longitud mayor o igual que cuatro tiene una cuerda. Se ve claramente a partir de esta definición que la clase de los grafos cordales es hereditaria.

Los grafos cordales tienen muchas aplicaciones prácticas, especialmente en el campo de la biología. Un buen ejemplo de esto son los árboles filogenéticos [19, 20], que son usados para modelar la evolución de especies, proteínas, etc.

Sin embargo, son las caracterizaciones de los grafos cordales las que nos interesan. Ellas aparecerán descritas a continuación.

La primera de ellas fue hallada por Dirac y es en términos de los separadores minimales de vértices.

Teorema 1.1. [4] Sea $G$ un grafo. Entonces, $G$ es cordal si y sólo si todo separador minimal de vértices de $G$ es completo.

Recordemos que un vértice $v$ de un grafo $G$ es simplicial si $N[v]$ es completo en $G$. Dirac fue capaz de utilizar la caracterización anterior para demostrar que todo grafo cordal tiene un vértice simplicial.

Teorema 1.2. [4] Sea $G$ un grafo cordal. Entonces, $G$ tiene un vértice simplicial. Si $G$ no es completo, entonces tiene dos vértices simpliciales no adyacentes.

Sea $v$ un vértice simplicial del grafo cordal $G$. Como la clase de los grafos cordales es hereditaria, $G-v$ también es cordal. Si $G-v$ tiene al menos un vértice, se puede aplicar el Teorema 1.2 nuevamente para concluir que tiene un vértice simplicial $w$. Consideremos ahora al grafo cordal $G-\{v, w\}$. El procedimiento anterior se puede repetir hasta que no queden más vértices. Esto nos motiva a introducir el concepto de orden de eliminación perfecto.

Un ordenamiento $v_{1} v_{2} \ldots v_{n}$ de los vértices de $G$ es un orden de eliminación perfecto si, para todo $1 \leq i \leq n, v_{i}$ es simplicial en $G_{i}:=G\left[\left\{v_{i}, \ldots, v_{n}\right\}\right]$. La segunda caracterización de los grafos cordales que vamos a ver es la siguiente: 
Teorema 1.3. [10] Sea $G$ un grafo. Entonces, $G$ es cordal si y sólo si posee un orden de eliminación perfecto.

Si se tiene en cuenta el comentario posterior al Teorema 1.2, es simple demostrar que todo grafo cordal tiene un orden de eliminación perfecto. No es difícil demostrar el recíproco.

Sean $v_{1} v_{2} \ldots v_{n}$ un orden de eliminación perfecto de $G$ y $C$ un ciclo de $G$ de longitud mayor o igual que cuatro. Sea $v_{i}$ el primer vértice de $C$ que aparece en el orden. Entonces, como $C$ es también un ciclo de $G_{i}$ y $v_{i}$ es simplicial en dicho grafo, los dos vértices adyacente a $v_{i}$ en $C$ inducen una cuerda.

La próxima caracterización de los grafos cordales nos mostrará que estos también pueden ser representados a través de árboles.

Teorema 1.4. [11] Sea $G$ un grafo. Entonces, $G$ es cordal si y sólo si $G$ es el grafo de intersección de una familia de subárboles de algún árbol $T$.

Podrá encontrarse un ejemplo de un grafo cordal y una representación suya como grafo de intersección de una familia de subárboles de un árbol en la Figura 1.1.
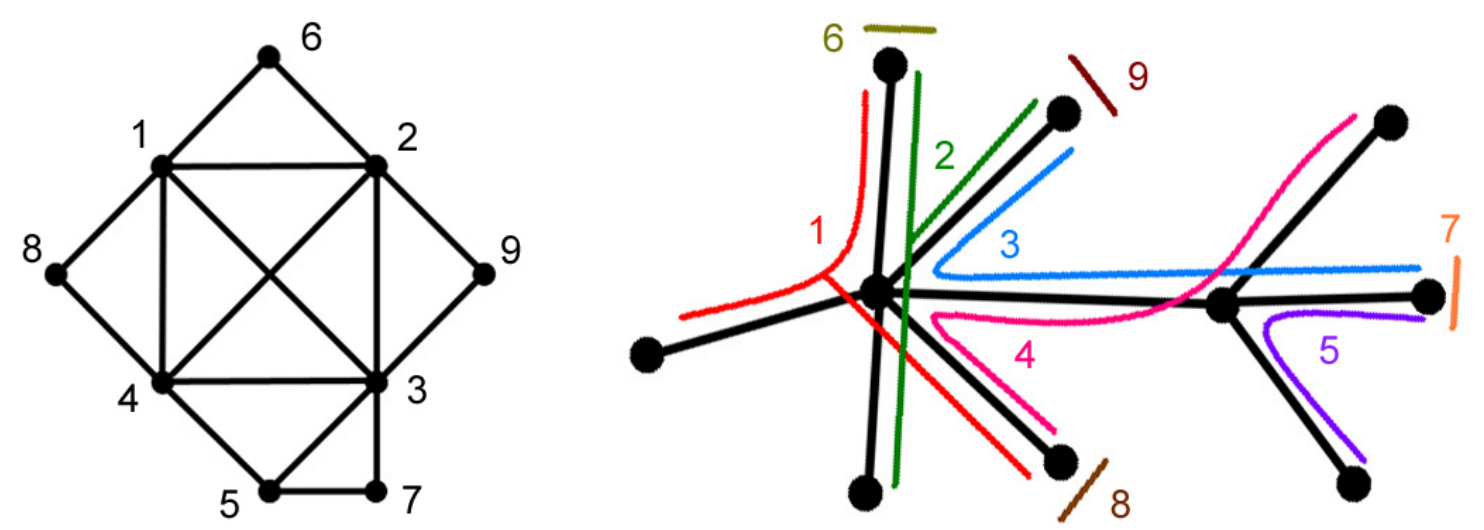

Figura 1.1: Un grafo cordal y una representación suya como grafo de intersección de una familia de subárboles de un árbol.

El árbol $T$ de la caracterización no es único. De hecho, se podrían agregar nuevos vértices para agrandar al árbol. Si la familia de subárboles no es modificada, entonces el grafo de intersección sigue siendo el mismo grafo cordal. Sin embargo, siempre es conveniente contar con un árbol con el mínimo número posible de vértices.

Sea $\left\{T_{i}\right\}_{1 \leq i \leq n}$ una familia de subárboles de $T$ y supongamos que su grafo de intersección es conexo.

Para cada vértice $v \in V(T)$ tal que, para todo $1 \leq i \leq n, v \notin V\left(T_{i}\right)$, removamos a $v$ de $T$. Al haberse supuesto que el grafo de intersección es conexo, lo que queda de $T$ es un subárbol suyo. Démosle a este subárbol el nombre $T^{\prime}$. Entonces, $\left\{T_{i}\right\}_{1 \leq i \leq n}$ es también una familia de subárboles de $T^{\prime}$ y el grafo de intersección de ella no cambia.

Existe un segundo procedimiento para reducir el número de vértices de $T$. Supongamos que existe una arista $x y$ en $T$ de modo que todo subárbol de la familia que tiene a $x$ como vértice también tiene a $y$ como vértice. En este caso, se procede a contraer a la arista $x y$ dejando solamente a $y$. Más precisamente, se define un nuevo árbol $T^{\prime \prime}$ tal que 
$V\left(T^{\prime \prime}\right)=V(T) \backslash\{x\}, N_{T^{\prime \prime}}[y]=\left(N_{T}[x] \cup N_{T}[y]\right) \backslash\{x\}$ y, para $z \in V\left(T^{\prime \prime}\right) \backslash\{y\}, N_{T^{\prime \prime}}[z]=N_{T}[z]$ si $x \notin N_{T}[z]$ y $N_{T^{\prime \prime}}[z]=\left(N_{T}[z] \cup\{y\}\right) \backslash\{x\}$ si $x \in N_{T}[z]$.

Si la arista $x y$ también es contraída en los subárboles de la familia que tienen a $x$ como vértice, no es complicado ver que se obtiene una familia de subárboles de $T^{\prime \prime}$ cuyo grafo de intersección se mantiene siendo el mismo.

Supongamos que hemos removido tantos vértices y contraído tantas aristas en $T$ como sea posible. Los subárboles de la familia resultante que contienen a un vértice $x$ dado claramente forman un conjunto completo del grafo de intersección. Más aun, ese conjunto completo es un clique y existe una correspondencia uno a uno entre los cliques del grafo de intersección y los vértices del árbol. Entonces, para cada vértice del grafo de intersección, el conjunto de cliques que lo contienen induce un subárbol, que justamente coincide con el subárbol de la familia que representa al vértice.

Un árbol clique de un grafo conexo $G$ es un árbol $T$ tal que $V(T)=\mathcal{C}(G)$ y, para todo $v \in V(G)$, el conjunto $\mathcal{C}_{v}$ de cliques de $G$ que contienen a $v$ induce un subárbol de $T$.

Existe una forma alternativa de definir a los árboles clique:

Proposición 1.5. Sean $G$ un grafo y $T$ un árbol tal que $V(T)=\mathcal{C}(G)$. Entonces, son equivalentes:

(a) $T$ es un árbol clique de $G$.

(b) Para todo $C_{1}, C_{2}, C_{3} \in \mathcal{C}(G), C_{3} \in T\left[C_{1}, C_{2}\right]$ implica que $C_{1} \cap C_{2} \subseteq C_{3}$.

Demostración. $(a) \Rightarrow(b)$. Sean $C_{1}, C_{2}, C_{3} \in \mathcal{C}(G)$ de modo que $C_{3} \in T\left[C_{1}, C_{2}\right]$ y sea $v$ un vértice de $C_{1} \cap C_{2}$. Entonces, $C_{1}$ y $C_{2}$ son elementos de $\mathcal{C}_{v}$. Como $\mathcal{C}_{v}$ induce un subárbol de $T$ y $C_{3} \in T\left[C_{1}, C_{2}\right], C_{3} \in \mathcal{C}_{v}$, es decir, $v \in C_{3}$. Por lo tanto, todo elemento de $C_{1} \cap C_{2}$ es un elemento de $C_{3}$, de lo cual se desprende la inclusión.

$(b) \Rightarrow(a)$. Sean $v$ un vértice de $G$ y $C_{1}, C_{2}$ cliques en $\mathcal{C}_{v}$. Entonces, $v \in C_{1} \cap C_{2}$. Sea $C_{3} \in T\left[C_{1}, C_{2}\right]$. Luego, $C_{1} \cap C_{2} \subseteq C_{3}$ y, por ende, $v \in C_{3}$, o sea, $C_{3} \in \mathcal{C}_{v}$. Por lo tanto, $T\left[\mathcal{C}_{v}\right]$ es un subgrafo conexo de $T$, es decir, $\mathcal{C}_{v}$ induce un subárbol de $T$.

Los árboles clique pueden también ser utilizados para caracterizar a los grafos cordales.

Teorema 1.6. [11] Sea $G$ un grafo conexo. Entonces, $G$ es cordal si y sólo si $G$ posee un árbol clique.

Idea de la demostración. Supongamos que $G$ es cordal. Entonces, por el Teorema 1.4, existe un árbol $T$ tal que $G$ es el grafo de intersección de una familia de subárboles de $T$. Se pueden usar las ideas que siguen al Teorema 1.4 para obtener un árbol clique a partir de $T$.

Recíprocamente, sea $T$ un árbol clique de $G$. Entonces, $G$ es el grafo de intersección de $\left\{T\left[\mathcal{C}_{v}\right]\right\}_{v \in V(G)}$. Por lo tanto, por el Teorema 1.4, G es cordal.

Se denotará por $\tau(G)$ a la familia de árboles clique de $G$.

El árbol clique es el modelo de representación arbórea que se utilizará aquí la mayoría de las veces. Esto se debe a que muchas propiedades estructurales de los grafos cordales se reflejan claramente en sus árboles clique, como se podrá ver luego. 

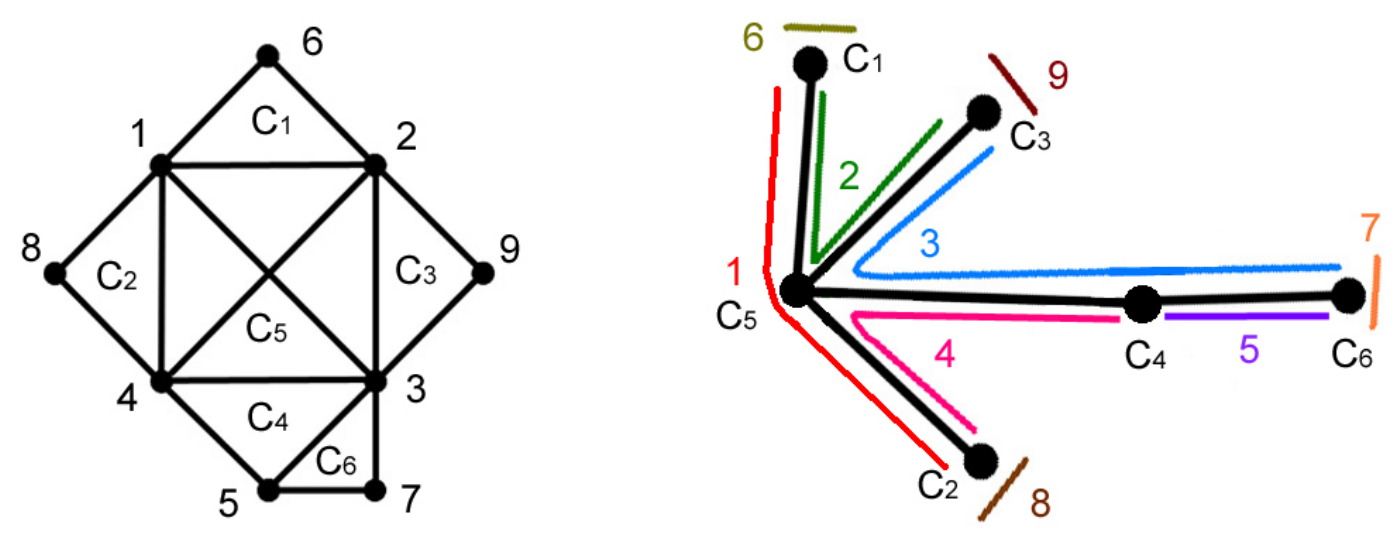

Figura 1.2: Luego de eliminar los vértices redundantes del árbol de la representación de la Figura 1.1, se obtiene un árbol clique.

\subsubsection{Subclases de los grafos cordales}

\section{Grafos UV, DV y RDV}

Es fácil definir subclases de los grafos cordales agregándole restricciones a los árboles y a las familias de subárboles utilizadas para representarlos como grafos de intersección.

Un grafo dirigido o digrafo es un grafo $G$ tal que, para toda arista de $G$, se define un extremo inicial y un extremo final. Si $e$ es una arista de $G$ cuyo extremo inicial es $u$ y cuyo extremo final es $v$, se dirá entonces que $e$ está orientada desde $u$ a $v$.

Un camino $v_{1} v_{2} \ldots v_{n}$ de un grafo dirigido es un camino dirigido si, para todo $1 \leq i \leq n-1$, la arista $v_{i} v_{i+1}$ está orientada desde $v_{i}$ a $v_{i+1}$.

Un árbol dirigido $T$ se dice enraizado si existe un vértice $v$ tal que, para todo $w \in V(T)$, $T\langle v, w\rangle$ es un $v w$-camino dirigido. A dicho vértice $v$ se lo llama raíz.

Sea $G$ un grafo cordal. $G$ es un grafo $U V$ si puede ser representado como el grafo de intersección de una familia de caminos de algún árbol. $G$ es un grafo $D V$ si puede ser representado como el grafo de intersección de una familia de caminos dirigidos de algún árbol dirigido. $G$ es un grafo $R D V$ si puede ser representado como el grafo de intersección de una familia de caminos dirigidos de un árbol dirigido enraizado.

Las tres clases son claramente hereditarias, siendo los grafos $R D V$ una subclase de los grafos $D V$, y siendo a su vez los grafos $D V$ una subclase de los grafos $U V$.

Dado el modo en que los grafos $U V, D V$ y $R D V$ fueron definidos, ellos también pueden ser caracterizados en términos de los árboles clique.

Teorema 1.7. [21] Sea $G$ un grafo conexo. Entonces,

(1) $G$ es $U V$ si y sólo si existe un árbol $T$ tal que $V(T)=\mathcal{C}(G)$ y, para todo $v \in V(G), \mathcal{C}_{v}$ induce un camino de $T$.

(2) $G$ es $D V$ si y sólo si existe un árbol dirigido $T$ tal que $V(T)=\mathcal{C}(G)$ y, para todo $v \in V(G)$, $\mathcal{C}_{v}$ induce un camino dirigido de $T$.

(3) $G$ es $R D V$ si y sólo si existe un árbol dirigido enraizado $T$ tal que $V(T)=\mathcal{C}(G)$ y, para todo $v \in V(G), \mathcal{C}_{v}$ induce un camino dirigido de $T$. 
Un árbol como el que es mencionado en (1) recibe el nombre de $U V$ árbol clique. Similarmente, los árboles en (2) y (3) reciben los respectivos nombres de $D V$ árbol clique y $R D V$ árbol clique.

El Teorema 1.7 puede ser aprovechado para mostrar que los grafos cordales, $U V, D V$ y $R D V$ constituyen clases distintas.

Se define un $k$-sol, para todo $k \geq 3$, como el grafo cuyo conjunto de vértices es $\left\{v_{1}, v_{2}, \ldots, v_{k}, w_{1}\right.$ $\left.w_{2}, \ldots, w_{k}\right\}$, de modo que $\left\{v_{1}, v_{2}, \ldots, v_{k}\right\}$ es completo, $N\left[w_{i}\right]=\left\{v_{i}, v_{i+1}, w_{i}\right\}, i=1,2, \ldots, k-1, \mathrm{y}$ $N\left[w_{k}\right]=\left\{v_{1}, v_{k}, w_{k}\right\}$. Un sol par es un sol tal que $k$ es par. Similarmente, se define al sol impar.
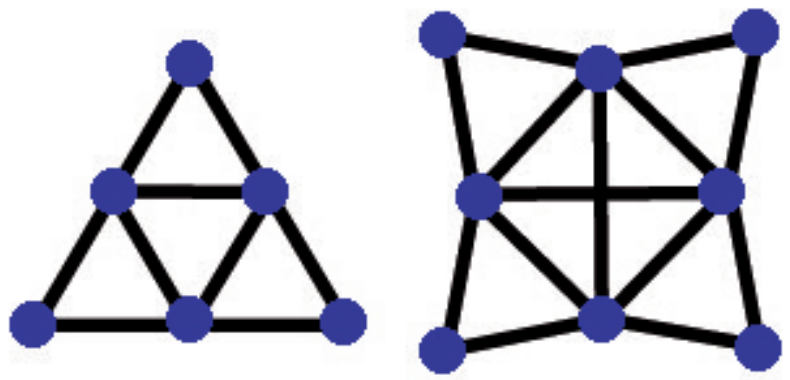

Figura 1.3: Un 3-sol y un 4-sol.

Los soles son grafos cordales. La familia de cliques de un $k$-sol tiene $k+1$ miembros $C, C_{1}, \ldots, C_{k}$, siendo $C=\left\{v_{1}, v_{2}, \ldots, v_{k}\right\}$ y $C_{i}=N\left[w_{i}\right], i=1,2, \ldots, k$.

Demostremos ahora que el $k$-sol posee un único árbol clique.

Sea $T$ un árbol clique de el $k$-sol. Como, por definición de árbol clique, todo $\mathcal{C}_{v_{i}}$ induce un subárbol de $T$, las intersecciones $\mathcal{C}_{v_{i}} \cap \mathcal{C}_{v_{i+1}}, i=1, \ldots, k-1$, y $\mathcal{C}_{v_{1}} \cap \mathcal{C}_{v_{k}}$ inducen también cada una un subárbol de $T$. Luego, $C C_{i}$ es una arista de $T, i=1, \ldots, k$.

Se concluye de esto que el único árbol clique del $k$-sol es aquel en el que $C$ es un vértice universal. Es simple ver que ese árbol es también un $U V$ árbol clique. Por lo tanto, los soles son grafos $U V$. Sin embargo, el 3 -sol no es $D V$ porque su árbol clique no puede ser dirigido de manera que se obtenga un $D V$ árbol clique. Más generalmente, ningún sol impar es un grafo $D V$; pero no es difícil verificar que los soles pares sí son grafos $D V$.

Demostremos ahora que el 4-sol no es un grafo $R D V$. Sea $T$ el árbol clique del 4-sol. Necesitamos probar que $T$ no puede ser transformado en un $R D V$ árbol clique.

La raíz de $T$ no puede ser $C$ porque, si lo fuera, $\mathcal{C}_{v_{i}}$ no induciría un camino dirigido de $T$, $i=1,2,3,4$.

Tampoco puede ser $C_{1}$ la raíz de $T$ porque, en ese caso, ni $\mathcal{C}_{v_{3}}$ ni $\mathcal{C}_{v_{4}}$ inducirían un camino dirigido de $T$. De manera análoga, $C_{i}$ no puede ser una raíz, $i=2,3,4$. Luego, $T$ no puede ser enraizado apropiadamente, por lo que el 4-sol no es un grafo $R D V$.

Más generalmente, ningún sol es un grafo $R D V$.

Sólo nos resta encontrar una diferencia entre las clases de los grafos cordales y los grafos $U V$. Sea $G$ un grafo tal que $V(G)=V^{\prime} \cup\{v\}$, de modo que $V^{\prime}$ induce un 3-sol en $G$ y $v$ es un vértice universal de $G$. El número de cliques de $G$ es el mismo que el de un 3-sol y estos dos grafos poseen también el mismo árbol clique. Entonces, $\mathcal{C}_{v}$ no induce un camino del único árbol clique de $G$, por lo que $G$ es un grafo cordal que no es $U V$. 


\section{Grafos cordal-potentes}

Un grafo $G$ es cordal-potente si es cordal y todas sus potencias también lo son.

Esta definición puede ser simplificada.

Proposición 1.8. [7] Sean $G$ un grafo y $k$ un número natural tal que $G^{k}$ es cordal. Entonces, $G^{k+2}$ es también cordal.

Se puede inferir de la Proposición 1.8 que $G$ es cordal-potente si y sólo si $G$ y $G^{2}$ son cordales. En consecuencia, existe un orden de eliminación perfecto para $G$ y un orden de eliminación perfecto para $G^{2}$. Más aun, esos órdenes pueden ser elegidos de manera que sean iguales.

Un vértice $v$ de $G$ es $p$-simplicial si es simplicial tanto en $G$ como en $G^{2}$. Los grafos cordalpotentes poseen una propiedad cuyo enunciado es muy similar al del Teorema 1.2.

Teorema 1.9. [3] Sea $G$ un grafo cordal-potente. Entonces, $G$ es completo o $G$ posee dos vértices p-simpliciales que no son adyacentes.

Como hemos visto, el Teorema 1.2 posibilita probar la existencia de los órdenes de eliminación perfectos en los grafos cordales. El Teorema 1.9 puede ser usado de manera similar. Un ordenamiento $v_{1} v_{2} \ldots v_{n}$ de los vértices de $G$ es un orden p-perfecto si, para todo $1 \leq i \leq n, v_{i}$ es p-simplicial en $G_{i}$. Entonces:

Teorema 1.10. [3] Sea $G$ un grafo. Entonces, son equivalentes:

- $G$ es cordal-potente.

- $G$ y $G^{2}$ son cordales.

- G posee un orden p-perfecto.

\section{Grafos fuertemente cordales}

Se dice que un grafo es fuertemente cordal si es cordal y todo ciclo par suyo de longitud mayor o igual que seis posee una cuerda que une a dos vértices a distancia impar en el ciclo. Una cuerda con esas características recibe el nombre de cuerda fuerte.

Se deduce de esta definición que la clase de grafos fuertemente cordales es hereditaria tal como lo es la de los grafos cordales. Otra cosa que estas dos clases tienen en común es que pueden caracterizarse mediante órdenes de eliminación.

Un vértice $v$ de un grafo $G$ es simple si el conjunto $\{N[u]: u \in N[v]\}$ está totalmente ordenado por inclusión. Todo vértice simple es simplicial, lo cual se demuestra a continuación. Sean $u_{1}$ y $u_{2}$ dos vértices en $N[v]$. Entonces, $N\left[u_{1}\right] \subseteq N\left[u_{2}\right]$ o $N\left[u_{2}\right] \subseteq N\left[u_{1}\right]$. Luego, $u_{1} \in N\left[u_{2}\right]$ o $u_{2} \in N\left[u_{1}\right]$. En cualquiera de los dos casos se infiere que $u_{1}$ y $u_{2}$ son vecinos. Por lo tanto, $N[v]$ es un conjunto completo, es decir, $v$ es simplicial.

Un ordenamiento $v_{1} v_{2} \ldots v_{n}$ de los vértices de $G$ es un orden de eliminación simple si, para todo $1 \leq i \leq n, v_{i}$ es simple en $G_{i}$. Entonces, como consecuencia de lo que se exhibe en el párrafo anterior, un orden de eliminación simple es un caso especial de orden de eliminación perfecto. Dicho esto, la primera caracterización de los grafos fuertemente cordales que se da es la siguiente:

Teorema 1.11. [8] Un grafo $G$ es fuertemente cordal si y sólo si posee un orden de eliminación simple. 
En su condición de clase hereditaria, los grafos fuertemente cordales pueden caracterizarse también a través de grafos prohibidos minimales.

De todos los grafos que se han considerado hasta ahora, los soles son un ejemplo de grafos cordales que no son fuertemente cordales. Si los vértices de un $k$-sol son nombrados como cuando este grafo fue definido, entonces $v_{1} w_{1} v_{2} w_{2} \ldots v_{i} w_{i} \ldots v_{k} w_{k} v_{1}$ es un ciclo par de longitud al menos seis sin cuerdas fuertes. Alternativamente, se puede verificar que todo sol no es fuertemente cordal porque no posee vértices simples. Conocer esto es de utilidad para obtener la próxima caracterización.

Teorema 1.12. [8] Un grafo $G$ es fuertemente cordal si y sólo si es cordal y sin soles inducidos.

\subsection{Grafos dualmente cordales}

Un grafo es dualmente cordal si es el grafo clique de algún grafo cordal. A diferencia de los grafos cordales, que casi siempre son definidos inicialmente en los cursos y libros en términos de ciclos y de cuerdas, los grafos dualmente cordales poseen varias definiciones y ninguna se destaca por sobre las demás, ajustándose el uso de éstas a las necesidades y objetivos de cada persona que trabaje con esta clase de grafos.

No hay ningún motivo especial para haber comenzado en este trabajo con la definición que usa a los grafos clique. Simplemente se optó por ella debido al hecho de que puede ser enunciada sin que se requiera introducir nuevos conceptos.

Comenzaremos esta sección con información acerca de la conexión entre los grafos dualmente cordales y los órdenes de vértices.

Sean $v$ y $w$ vértices de un grafo $G$. Se dice que $w$ es un máximo vecino de $v$ si $N^{2}[v] \subseteq N[w]$. No se descarta para esta definición el caso de que $v$ y $w$ sean iguales. Si $v$ es un máximo vecino de sí mismo, entonces todo vértice a distancia a lo sumo dos de $v$ está a distancia a lo sumo uno de $v$. Luego, si $G$ es conexo, $v$ es un vértice universal.

Un ordenamiento $v_{1} v_{2} \ldots v_{n}$ de los vértices de $G$ es un orden de vecindades máximas si, para todo $1 \leq i \leq n, v_{i}$ tiene un máximo vecino en $G_{i}$.

Esta definición permite enunciar la siguiente caracterización de los grafos dualmente cordales.

Teorema 1.13. [3] Sea $G$ un grafo. Entonces, $G$ es dualmente cordal si y sólo si posee un orden de vecindades máximas.

Es fácil encontrar grafos que no son dualmente cordales y corroborarlo mediante el uso de esta caracterización. Por ejemplo, el ciclo de longitud cuatro no es dualmente cordal porque ninguno de sus vértices posee un máximo vecino. Lo mismo vale para los soles. Sin embargo, el grafo que consiste de un ciclo inducido de longitud cuatro más un vértice universal es dualmente cordal.

Más generalmente, todo grafo con un vértice universal $u$ es dualmente cordal. De hecho, todo vértice del grafo tiene a $u$ como máximo vecino. Por ende, cualquier ordenamiento de los vértices del grafo que termine en $u$ es un orden de vecindades máximas.

Como consecuencia de lo expuesto en los párrafos anteriores, se infiere que la clase de los grafos dualmente cordales no es hereditaria.

Veremos ahora que los grafos dualmente cordales tienen asociada una estructura análoga al árbol clique de los grafos cordales.

Teorema 1.14. [3] Sea $G$ un grafo conexo. Son equivalentes:

1. G es dualmente cordal. 


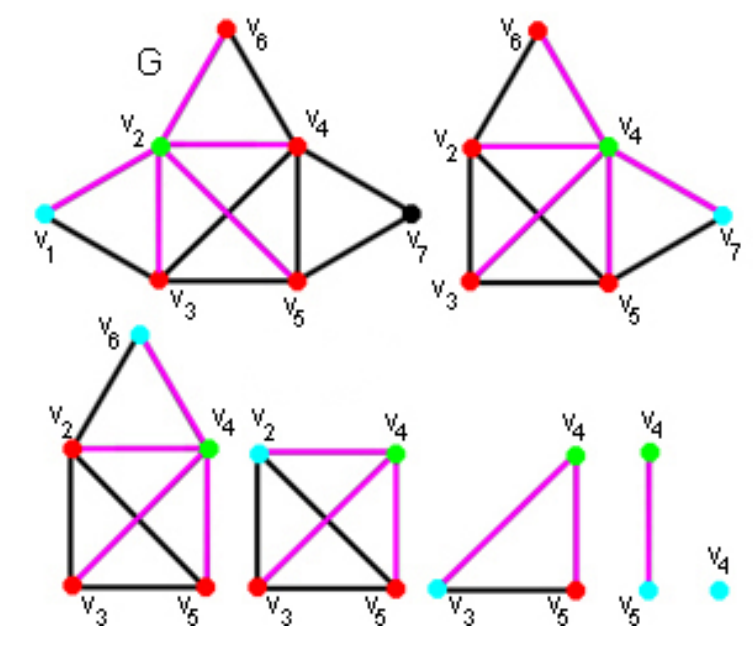

Figura 1.4: $v_{1} v_{7} v_{6} v_{2} v_{3} v_{5} v_{4}$ es un orden de vecindades máximas de $G$. Se muestran en la figura las etapas de la obtención de dicho orden. Los vértices que aparecen en celeste son los que deben agregarse al orden, siendo los verdes sus máximos vecinos. El conjunto de vértices coloreados conforma el disco centrado en el vértice celeste de radio 2. Las aristas violetas ayudan a discernir que el vértice verde es realmente un máximo vecino.

2. G posee un árbol generador $T$ tal que todo clique de $G$ induce un subárbol de $T$.

3. G posee un árbol generador $T$ tal que la vecindad cerrada de todo vértice de $G$ induce un subárbol de $T$.

4. G posee un árbol generador $T$ tal que todo disco de $G$ induce un subárbol de $T$.

Vale incluso que todo árbol que se sabe que satisface alguna de las condiciones mencionadas en 2., 3. y 4. también satisface las otras dos. Diremos que un árbol así es compatible con $G$.

La caracterización de los grafos conexos dualmente cordales dada por 3. puede ser reescrita de la siguiente manera:

Teorema 1.15. [13] Un grafo conexo $G$ es dualmente cordal si y sólo si $G$ posee un árbol generador $T$ de modo que, para todo $x y \in E(G)$ y todo $z$ en el $x y$-camino de $T, x z \in E(G)$ e $y z \in E(G)$.

Nos encontramos ahora en una mejor posición para entender por qué los grafos dualmente cordales son llamados de esa manera. El Teorema 1.14 implica que un grafo $G$ es dualmente cordal si y sólo si $\mathcal{C}(G)$ puede ser representada como una familia de subárboles de un árbol. Por otro lado, el Teorema 1.6 implica que $G$ es cordal si y sólo si la familia dual $\operatorname{de~} \mathcal{C}(G)$ puede ser representada como una familia de subárboles de un árbol.

Finalmente, se incluye otra caracterización de los grafos dualmente cordales. Ésta también involucra a los cliques.

Teorema 1.16. [6] Un grafo $G$ es dualmente cordal si y sólo si $G$ es clique-Helly y $K(G)$ es cordal. 

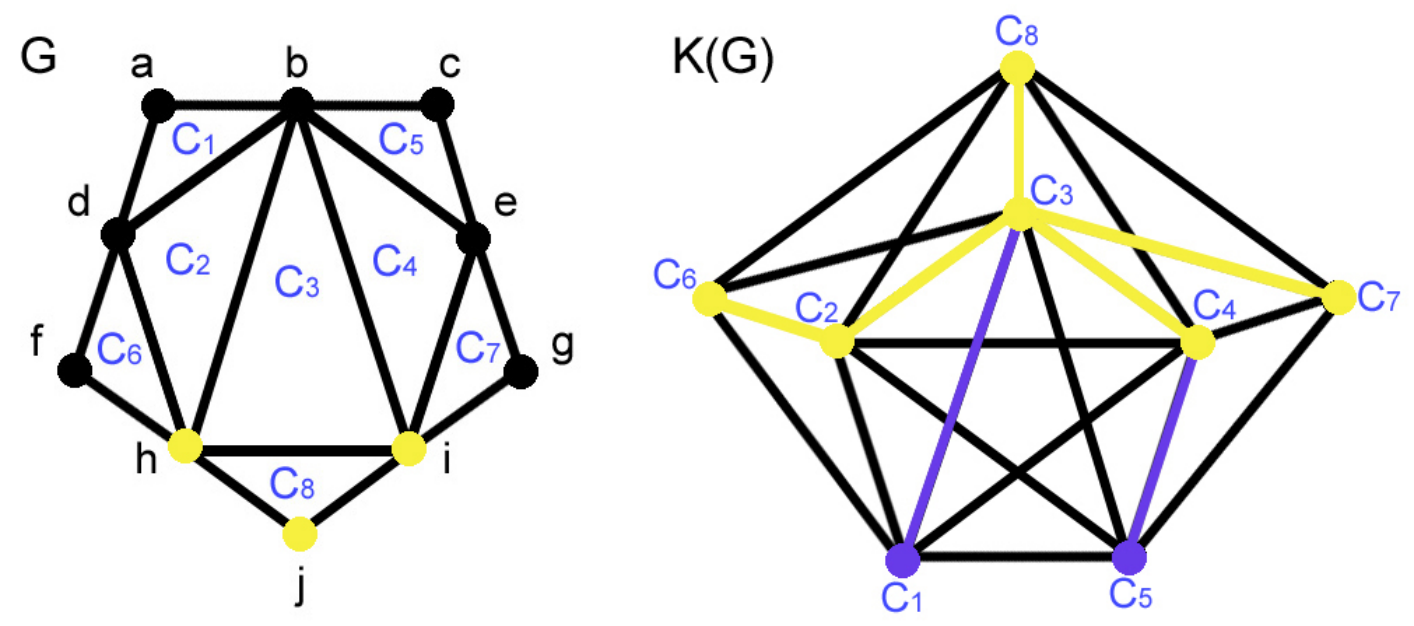

Figura 1.5: Un grafo cordal $G$ y su grafo clique. En color, las aristas de un árbol compatible de $K(G)$. El amarillo corresponde al subárbol inducido por $N_{K(G)}\left[C_{8}\right]$.

\subsubsection{Subclases de los grafos dualmente cordales}

\section{Grafos doblemente cordales}

Un grafo $G$ es doblemente cordal si es tanto cordal como dualmente cordal. Como tal, debe poseer un orden de eliminación perfecto y un orden de vecindades máximas.

Un vértice $v$ es doblemente simplicial si es simplicial y tiene un máximo vecino. Un ordenamiento $v_{1} v_{2} \ldots v_{n}$ de los vértices de $G$ es doblemente perfecto si, para todo $1 \leq i \leq n, v_{i}$ es doblemente simplicial en $G_{i}$. Podemos hacer uso de un orden así para caracterizar a los grafos doblemente cordales.

Teorema 1.17. [3] Un grafo $G$ es doblemente cordal si y sólo si $G$ posee un orden doblemente perfecto.

\section{Grafos fuertemente cordales}

Sean $G$ un grafo y $v$ un vértice simple de él. Por definición, sabemos que el conjunto $\{N[u]: u \in N[v]\}$ está totalmente ordenado por inclusión. Entonces, existe un vértice $w$ tal que $N[u] \subseteq N[w]$ para todo $u \in N[v]$. Luego, $w$ es un máximo vecino de $v$.

Se concluye a partir de un razonamiento de este tipo que todo orden de eliminación simple es un orden de vecindades máximas. Por lo tanto, los grafos fuertemente cordales constituyen una subclase de la de los grafos dualmente cordales. Más aun, todo orden de eliminación simple es doblemente perfecto, por lo que los grafos fuertemente cordales también están contenidos en la clase de grafos doblemente cordales.

Existe una conexión aun más fuerte entre los grafos fuertemente cordales y los dualmente cordales, que se deduce de las dos proposiciones que siguen.

Proposición 1.18. [13] Sea $G$ un grafo cordal que no es clique-Helly. Entonces, G posee al 3-sol como subgrafo inducido.

Proposición 1.19. [13] Sea $G$ un grafo cordal y clique-Helly tal que $K(G)$ no es cordal. Entonces, para algún $k \geq 4, G$ tiene al $k$-sol como subgrafo inducido. 
Como consecuencia del Teorema 1.16 y la caracterización de los grafos fuertemente cordales por subgrafos inducidos prohibidos, se concluye a partir de las últimas dos proposiciones que todo subgrafo inducido de un grafo fuertemente cordal es dualmente cordal. Luego, los grafos fuertemente cordales son grafos dualmente cordales hereditarios.

Además, los ciclos de longitud mayor o igual que cuatro y los soles no son grafos dualmente cordales, por lo que ninguno de ellos puede ser subgrafo inducido de un grafo dualmente cordal hereditario. Luego, se desprende la siguiente caracterización:

Teorema 1.20. Un grafo $G$ es fuertemente cordal si y sólo si es un grafo dualmente cordal hereditario.

\section{Grafos dualmente DV y dualmente RDV}

Dado que los grafos dualmente cordales pueden ser definidos como los grafos clique de los grafos cordales, es a priori posible que la imagen por el operador clique de ciertas subclases de los grafos cordales dé lugar a nuevas subclases de los grafos dualmente cordales.

Esto no es cierto en muchos casos. Por ejemplo, K(DOBLEMENTE CORDALES $)=D O B L E$ MENTE CORDALES [3], K(FUERTEMENTE CORDALES $)=$ FUERTEMENTE CORDALES [1] Y $K(U V)=C O R D A L E S$ [25].

No obstante, sí obtenemos nuevas subclases cuando usamos a los grafos $D V$ y $R D V$.

Un grafo $G$ es dualmente $D V$ si es el grafo clique de algún grafo $D V$. Es dualmente $R D V$ si es el grafo clique de algún grafo $R D V$. Al igual que los grafos dualmente cordales, ambas clases poseen una estructura arbórea que las caracteriza.

Teorema 1.21. Sea $G$ un grafo conexo. Entonces:

(a) $G$ es dualmente $D V$ si y sólo si $G$ posee un árbol generador $T$ dirigible de modo que todo clique de $G$ induzca un camino dirigido de $T$.

(b) $G$ es dualmente $R D V$ si y sólo si $G$ posee un árbol generador $T$ dirigible y enraizable de modo que todo clique de $G$ induzca un camino dirigido de $T$.

Un árbol dirigido como el descrito en (a) recibe el nombre de $D V$ árbol compatible; y uno como el descrito en (b) recibe el nombre de $R D V$ árbol compatible.

Así como los soles pueden ser usados para establecer la diferencia entre los grafos cordales, $D V$ y $R D V$, los grafos clique de los soles nos permiten diferenciar a sus clases duales.

La rueda $W_{n}, n \geq 4$, es un grafo tal que $V\left(W_{n}\right)=\left\{u, v_{1}, \ldots, v_{n}\right\}$, donde $\left\{v_{1}, \ldots, v_{n}\right\}$ induce un ciclo sin cuerdas y $u$ es un vértice universal. Es fácil comprobar que, para todo $k \geq 4$, el grafo clique del $k$-sol es $W_{k}$.

Usando el Teorema 1.21, se puede verificar que, para todo $k$ impar, $W_{k}$ es dualmente cordal pero no dualmente $D V$ y, para todo $k$ par, $W_{k}$ es dualmente $D V$ pero no dualmente $R D V$. 


\section{Capítulo 2}

\section{Vértices excéntricos especiales en grafos cordales, dualmente cordales y sus subclases}

En este capítulo, se encontrarán analogías entre los ordenamientos en los grafos cordales, doblemente cordales, fuertemente cordales, cordal-potentes y dualmente cordales y entre los vértices que los distinguen. Esto se hará principalmente a través del estudio de los vértices excéntricos, como se verá en los Teoremas 2.3, 2.7, 2.9, 2.10 y 2.13.

En primer lugar, notemos que los vértices simpliciales y los vértices con máximos vecinos suelen ser vértices dominados. Nos concentraremos a partir de ahora en aspectos métricos de los grafos, por lo que la siguiente proposición es de nuestro interés:

Proposición 2.1. Sean $G$ un grafo y $u, v, w, x$ vértices de $G$ tales que $w$ es diferente a los otros tres y $w$ está dominado por $x$. Entonces, $d_{G-w}(u, v)=d_{G}(u, v)$.

Demostración. Si no existe ningún uv-camino en $G$, entonces $d_{G-w}(u, v)=d_{G}(u, v)=\infty$.

En caso contrario, sea $P$ un $u v$-camino en $G$ con longitud mínima. Si $w$ no es un vértice de $P$, entonces $P$ es también un camino en $G-w$ y, por ende, $d_{G-w}(u, v)=d_{G}(u, v)$.

Si $w$ es un vértice de $P$, sea $y_{1} \ldots y_{i} w y_{i+1} \ldots y_{j}$ el orden de los vértices en $P$, de modo que $y_{1}=u$ e $y_{j}=v$. Demostremos ahora que $x$ no es un vértice de $P$. Como $w$ está dominado por $x$, $y_{i}, y_{i+1} \in N[x]$. Si existe $k$ tal que $k \leq i$ e $y_{k}=x$, entonces $y_{1} \ldots y_{k} y_{i+1} \ldots y_{j}$ es un $u v$-camino que es más corto que $P$, lo cual es absurdo. Por lo tanto, para todo $1 \leq k \leq i, y_{k} \neq x$. Similarmente, para todo $i+1 \leq k \leq j, y_{k} \neq x$.

Se infiere de todo lo anterior que $y_{1} \ldots y_{i} x y_{i+1} \ldots y_{j}$ es un $u v$-camino en $G-w$ que tiene la misma longitud que $P$. Luego, la igualdad $d_{G-w}(u, v)=d_{G}(u, v)$ se cumple.

Hay algo más que se puede demostrar acerca de la remoción de vértices dominados. Aunque no todas las clases que se han mencionado hasta ahora son hereditarias, puede verse que todas ellas son cerradas bajo la operación de remover vértices dominados.

Proposición 2.2. Sean $G$ un grafo cordal/dualmente cordal/doblemente cordal/cordal-potente/ fuertemente cordal y $v$ un vértice de $G$ dominado por otro vértice $w$. Entonces, $G-v$ es cordal/dualmente cordal/doblemente cordal/cordal-potente/fuertemente cordal. 
Demostración. Como las clases de grafos cordales y fuertemente cordales son hereditarias, la prueba es directa si $G$ es cordal o si $G$ es fuertemente cordal. Consideremos ahora los casos restantes:

$G$ es dualmente cordal: Sea $C$ un clique de $G-v$. Definamos a $C^{\prime}$ de manera que $C^{\prime}=C \cup\{v\}$ si $C \subseteq N_{G}[v]$ y $C^{\prime}=C$ en caso contrario. Es simple ver que $C^{\prime}$ es un clique de $G$.

Probemos ahora que $G-v$ es clique-Helly y que $K(G-v)$ es cordal.

Sean $C_{1}, C_{2}, \ldots, C_{n}$ cliques de $G-v$ que se intersecan de a pares. Entonces, $C_{1}^{\prime}, C_{2}^{\prime}, \ldots, C_{n}^{\prime}$ son cliques de $G$ que se intersecan de a pares. Como $G$ es dualmente cordal, $G$ es clique-Helly, por lo que se puede tomar un vértice $u \in C_{1}^{\prime} \cap C_{2}^{\prime} \cap \ldots \cap C_{n}^{\prime}$. Si $u \neq v$, entonces $u \in C_{1} \cap C_{2} \cap \ldots \cap C_{n}$. Si $u=v$, entonces, para todo $1 \leq i \leq n, w \in C_{i}^{\prime}$, ya que $v$ está dominado por $w$. Por lo tanto, $w \in C_{1} \cap C_{2} \cap \ldots \cap C_{n}$.

Luego, $G-v$ es clique-Helly.

Se deduce del razonamiento anterior que, dados $C_{1}, C_{2} \in \mathcal{C}(G-v), C_{1} \cap C_{2} \neq \emptyset$ si y sólo si $C_{1}^{\prime} \cap C_{2}^{\prime} \neq \emptyset$. Por lo tanto, $K(G-v)$ es un subgrafo inducido de $K(G)$. Debido a que $G$ es dualmente cordal, se sabe que $K(G)$ es cordal. Luego, $K(G-v)$ es cordal.

Por el Teorema 1.16, $G-v$ es dualmente cordal.

$G$ es doblemente cordal: Ya sabemos de lo anterior que $G-v$ es cordal y también dualmente cordal. Luego, $G-v$ es doblemente cordal.

$G$ es cordal-potente: Sabemos que $G-v$ es cordal. Veamos ahora que $(G-v)^{2}=G^{2}-v$.

Es claro que toda arista de $(G-v)^{2}$ es también una arista de $G^{2}-v$. Para probar lo recíproco, basta con aplicar la Proposición 2.1.

Entonces, $(G-v)^{2}$ es un subgrafo inducido de $G^{2}$, el cual es cordal. Por lo tanto, $(G-v)^{2}$ es cordal.

Luego, $G-v$ es cordal-potente.

Volvamos a los grafos cordales. Como se ha visto en el Capítulo 1, la existencia de un orden de eliminación perfecto en un grafo cordal es una consecuencia directa del Teorema 1.2. El Teorema 1.2 es un resultado clásico en la historia de los grafos cordales. Pero luego se demostraron otras propiedades que son más específicas a la hora de establecer en qué parte del grafo se puede encontrar un vértice simplicial. Por ejemplo:

Teorema 2.3. [9] Sean $G$ un grafo cordal y $v \in V(G)$. Entonces, $v$ posee un vértice excéntrico que es simplicial.

Demostración. Se incluye una demostración debido a la importancia de este teorema en el desarrollo del capítulo.

La demostración es trivial si $G$ posee un vértice únicamente, por lo que supondremos que $G$ tiene al menos dos vértices.

Se probará primero que existe un orden de eliminación perfecto de $G$ tal que $v$ es su último vértice.

Si $G$ es completo, entonces cualquier orden de los vértices de $G$ es perfecto. En particular, lo es uno que termine en $v$.

Si $G$ no es completo, existen entonces dos vértices simpliciales $v_{1}$ y $v_{2}$ no adyacentes. Se puede suponer sin pérdida de generalidad que $v \neq v_{1}$. Entonces, hacemos que $v_{1}$ sea el primer vértice del orden de eliminación perfecto. Para obtener el segundo vértice del orden, se repite el mismo razonamiento, esta vez sobre el grafo $G-v_{1}$. Luego, se continúa removiendo los vértices obtenidos, siempre utilizando el mismo criterio, hasta que el orden de eliminación perfecto quede completo. 
Sean $v_{1} \ldots v_{n-1} v_{n}, v_{n}=v$, el orden obtenido y $v_{j}$ el vértice excéntrico de $v$ que aparece primero en el orden. Probaremos ahora que $v_{j}$ es simplicial en $G$.

Supongamos que $v_{j}$ no es simplicial en $G$. Entonces, existen dos vértices no adyacentes $x$ e $y$ en $N\left[v_{j}\right]$. Como $v_{j}$ es simplicial en $G_{j}, x \notin V\left(G_{j}\right)$ o $y \notin V\left(G_{j}\right)$. Supongamos sin pérdida de generalidad que $x \notin V\left(G_{j}\right)$ y que $x=v_{i}$, para algún $i<j$. Dado que $v_{i}$ es simplicial en $G_{i}$, la vecindad cerrada de $v_{i}$ en $G_{i}$ está contenida en la vecindad cerrada de $v_{j}$ en $G_{i}$. Entonces, la distancia entre $v_{i}$ y $v$ en $G_{i}$ es mayor o igual que la distancia entre $v_{j}$ y $v$ en $G_{i}$. Podemos concluir, aplicando la Proposición 2.1 tantas veces como sea necesario, que la distancia entre $v_{i}$ y $v$ en $G$ es mayor o igual que la distancia entre $v_{j}$ y $v$ en $G$.

Por lo tanto, como $v_{j}$ es excéntrico de $v$ en $G, v_{i}$ es también excéntrico, lo cual contradice la manera en que $v_{j}$ fue elegido.

Luego, $v_{j}$ es simplicial.

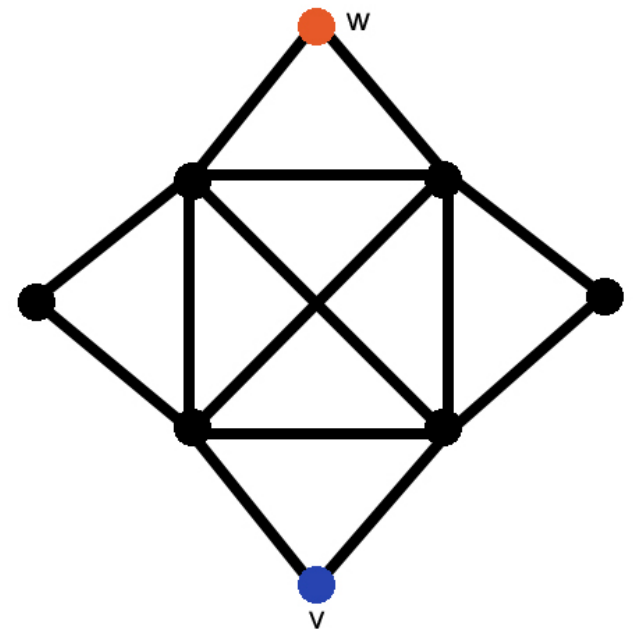

Figura 2.1: Un 4-sol, un vértice $v$ y un vértice simplicial $w$ que es excéntrico de $v$.

Es interesante ver que el Teorema 2.3 implica el Teorema 1.2.

El Teorema 2.3 claramente implica la existencia de un vértice simplicial. Sea $v$ un vértice simplicial de $G$. Si $\operatorname{ecc}(v) \leq 1$, entonces $G$ es completo. Si $\operatorname{ecc}(v)>1$, sea $w$ un vértice simplicial de $G$ que es excéntrico de $v$. Entonces, $v$ y $w$ son dos vértices simpliciales no adyacentes.

El Teorema 2.3 constituye la base de este capítulo. Será de nuestro interés determinar si es posible encontrar un resultado análogo acerca de los grafos dualmente cordales. Más específicamente, dados un grafo dualmente cordal $G$ y $v \in V(G)$, nos preguntamos si $v$ posee un vértice excéntrico con máximo vecino. Esto resultará ser cierto y no demasiado complicado de demostrar. Como resultado, se desprenderán otras propiedades reminiscentes de algunas de las que aparecen en la literatura sobre grafos cordales. Probarlas requerirá de algunos resultados previos.

Lema 2.4. [3] Sean $G$ un grafo dualmente cordal y $A \subseteq V(G)$ de modo que d(x,y) $\leq 2$ para todo par de elementos $x$ e y de $A$. Entonces, existe un vértice $w$ tal que $A \subseteq N[w]$.

Lema 2.5. [3] Sea $G$ un grafo dualmente cordal. Entonces, $G^{2}$ es cordal. 
Lema 2.6. Sean $G$ un grafo dualmente cordal y $v$ un vértice simplicial de $G^{2}$. Entonces, $v$ tiene un máximo vecino en $G$.

Demostración. Como $v$ es simplicial en $G^{2}$, la distancia en $G$ entre todo par de vértices de $N_{G}^{2}[v]$ es menor o igual a dos. Entonces, por el Lema 2.4, existe un vértice $w$ tal que $N_{G}^{2}[v] \subseteq N_{G}[w]$. Por lo tanto, $w$ es un máximo vecino de $v$.

Se puede demostrar ahora el primer resultado de importancia.

Teorema 2.7. Sean $G$ un grafo dualmente cordal y $v$ un vértice de $G$. Entonces, existe un vértice excéntrico de $v$ con máximo vecino.

Demostración. Supongamos primero que $e c c_{G}(v)$ es impar y sea $v^{\prime}$ un vértice excéntrico de $v$ en $G$. Como $G^{2}$ es cordal, el Teorema 2.3 implica que existe un vértice $w$ que es simplicial en $G^{2}$ y es excéntrico de $v$ en $G^{2}$. Entonces, por el Lema 2.6, $w$ tiene un máximo vecino en $G$. Probemos ahora que $w$ es también excéntrico de $v$ en $G$.

Notar primero que, por la definición de $G^{2}$, si dos vértices están a distancia $k$ en $G$, la distancia en $G^{2}$ entre ellos es $\frac{k}{2}$ si $k$ es par o $\frac{k+1}{2}$ si $k$ es impar. Por lo tanto, como $\operatorname{ecc}_{G}(v)$ es impar, $d_{G^{2}}\left(v, v^{\prime}\right)=\frac{e c c_{G}(v)+1}{2}$. Además, todo vértice excéntrico de $v$ en $G$ es también excéntrico de $v$ en $G^{2}$. Entonces, $v^{\prime}$ es excéntrico de $v$ en $G^{2}$ y, por ende, la excentricidad de $v$ en $G^{2}$ es igual a $\frac{e c c_{G}(v)+1}{2}$. Ya que $w$ es también excéntrico de $v$ en $G^{2}$, se deduce que $d_{G^{2}}(v, w)=\frac{e c c_{G}(v)+1}{2}$.

Usando la definición de $G^{2}$ nuevamente y que $d_{G^{2}}(v, w)=\frac{e c c_{G}(v)+1}{2}$, se obtienen dos posibles valores para $d_{G}(v, w)$, a saber, $\operatorname{ecc}_{G}(v)$ y $e c c_{G}(v)+1$. Por lo tanto, $d_{G}(v, w) \geq \operatorname{ecc}_{G}(v)$. Además, la definición de excentricidad implica que $d_{G}(v, w) \leq e c c_{G}(v)$. Luego, $d_{G}(v, w)=e c c_{G}(v) \mathrm{y}$, por ende, $w$ es el vértice buscado.

Si $\operatorname{ecc}_{G}(v)$ es par, sea $G^{\prime}$ el grafo obtenido a partir de $G$ agregando un nuevo vértice $v^{*}$ y haciéndolo adyacente a $v$. Entonces, $G^{\prime}$ es dualmente cordal. De hecho, si $v_{1} \ldots v_{n}$ es un orden de vecindades máximas de $G, v^{*} v_{1} \ldots v_{n}$ es un orden de vecindades máximas de $G^{\prime}$. Como $\operatorname{ecc}_{G}(v)$ es par, se cumple que $e c c_{G^{\prime}}\left(v^{*}\right)$ es impar y, por el caso previo, existe un vértice $u$ con un máximo vecino en $G^{\prime}$ tal que $d_{G^{\prime}}\left(v^{*}, u\right)=e c c_{G^{\prime}}\left(v^{*}\right)$. Es claro que $u$ es excéntrico de $v$ en $G$. Veamos ahora que $u$ tiene un máximo vecino en $G$.

Sea $w$ un máximo vecino de $u$ en $G^{\prime}$. Si $w=v^{*}$, entonces $u=v$. Como $v^{*}$ es un máximo vecino de $v, v^{*}$ domina a $v$. Dada la forma en que $G^{\prime}$ fue construido, $v$ domina a $v^{*}$ también. Así, $N_{G^{\prime}}[v]=N_{G^{\prime}}\left[v^{*}\right]=\left\{v, v^{*}\right\}$. Se infiere que $v$ no es adyacente a ningún otro vértice de $G \mathrm{y}$ que, por ende, es un máximo vecino de sí mismo en $G$.

Si $w \neq v^{*}$, entonces $w \in V(G)$. La inclusión $N_{G^{\prime}}^{2}[u] \subseteq N_{G^{\prime}}[w]$ implica que $N_{G}^{2}[u] \subseteq N_{G}[w]$. Luego, $w$ es un máximo vecino de $u$ en $G$.

Si $\operatorname{ecc}_{G}(v)=\infty$, entonces $G$ no es conexo. Sea $w$ un vértice con máximo vecino en una componente conexa de $G$ diferente a la de $v$. De esta manera, $w$ es el vértice que se buscaba.

Así como hemos visto que el Teorema 2.3 puede ser usado para probar propiedades básicas de los grafos cordales, podemos ver ahora que el Teorema 2.7 tiene el mismo nivel de utilidad y nos permite encontrar conexiones entre los enfoques por los cuales los grafos cordales y dualmente cordales pueden ser estudiados.

Corolario 2.8. Sea $G$ un grafo dualmente cordal con más de un vértice. Entonces,

(a) G es completo o posee dos vértices no adyacentes tales que cada uno de ellos tiene un máximo vecino. 
(b) Existen dos vértices $v_{1}$ y $v_{2}$, ambos con máximo vecino, tales que $d\left(v_{1}, v_{2}\right)=\operatorname{diam}(G)$.

\section{Demostración.}

(a): Sea $v$ un vértice de $G$ con máximo vecino. Si $\operatorname{ecc}(v)=1$, entonces $v$ es universal y todo vértice de $G$ tiene a $v$ como máximo vecino. Por lo tanto, $G$ es completo o existen dos vértices no adyacentes que tienen a $v$ como máximo vecino. Si $\operatorname{ecc}(v)>1$, sea $w$ un vértice excéntrico de $v$ con máximo vecino. Entonces, $v$ y $w$ son dos vértices no adyacentes y cada uno tiene un máximo vecino.

(b): Sean $k=\operatorname{diam}(G)$ y $x, y$ dos vértices tales que $d(x, y)=k$. Entonces, existe un vértice $v_{1}$ con máximo vecino que es excéntrico de $x$, por lo que $d\left(x, v_{1}\right)=k$. Asimismo, existe un vértice $v_{2}$ con máximo vecino que es excéntrico de $v_{1}$. Luego, $d\left(v_{1}, v_{2}\right)=k$.

En lo que resta del capítulo, se realizarán estudios similares sobre grafos cordal-potentes, doblemente cordales y fuertemente cordales. Como queda claro qué nos proponemos, se procederá directamente a enunciar los teoremas y demostrarlos.

Teorema 2.9. Sean $G$ un grafo cordal-potente $y v \in V(G)$. Entonces, $v$ posee un vértice excéntrico que es p-simplicial.

Demostración. Como consecuencia del Teorema 1.9, existe un orden p-perfecto $v_{1} \ldots v_{n}$ de $G$ tal que $v_{n}=v$.

Supongamos primero que $\operatorname{ecc}_{G}(v)$ es impar.

Sea $v_{j}$ el primer vértice excéntrico de $v$ en aparecer en el orden. Como $v_{1} \ldots v_{n}$ es un orden de eliminación perfecto de $G$, podemos repetir los argumentos de la prueba del Teorema 2.3 para concluir que $v_{j}$ es simplicial en $G$.

Por construcción, $v_{1} \ldots v_{n}$ es también un orden de eliminación perfecto de $G^{2}$. Ya que $\operatorname{ecc}_{G}(v)$ es impar, $v_{j}$ es el primer vértice excéntrico de $v$ en $G^{2}$ que aparece en el orden. Se pueden aplicar los mismos argumentos de nuevo para concluir que $v_{j}$ es simplicial en $G^{2}$. Luego, $v_{j}$ es un vértice p-simplicial de $G$.

Si $\operatorname{ecc}_{G}(v)$ es par, se construye el grafo $G^{\prime}$ de la misma manera que en la demostración del Teorema 2.7. Entonces, $G^{\prime}$ es cordal-potente y $v^{*} v_{1} \ldots v_{n}$ es un orden de eliminación p-perfecto de $G^{\prime}$. También se verifica que $\operatorname{ecc}_{G^{\prime}}\left(v^{*}\right)$ es impar.

Se puede obtener, utilizando la primera parte de esta demostración, un vértice $w$ que es p-simplicial y excéntrico de $v^{*}$ en $G^{\prime}$. Por la Proposición 2.1, las distancias entre vértices de $G$ son las mismas en $G$ y en $G^{\prime}$. Se infiere de esto que $w$ es también p-simplicial en $G$. Finalmente, es claro que $w$ es un vértice excéntrico de $v$ en $G$.

Si $\operatorname{ecc}_{G}(v)=\infty$, se puede tomar un vértice p-simplicial en una componente conexa de $G$ diferente de la de $v$ y claramente resultará ser excéntrico de $v$.

Teorema 2.10. Sean $G$ un grafo doblemente cordal y $v \in V(G)$. Entonces, existe un vértice excéntrico de $v$ que es doblemente simplicial.

Demostración. Como $G$ es dualmente cordal, $G^{2}$ es cordal según el Lema 2.5. Por lo tanto, $G$ es cordal-potente. Por el Teorema 2.9, existe un vértice $w$ que es simplicial tanto en $G$ como en $G^{2}$ y que es excéntrico de $v$. Por el Lema 2.6, $w$ posee un máximo vecino en $G$, por lo que $w$ es un vértice doblemente simplicial.

Corolario 2.11. Sea $G$ un grafo cordal-potente/doblemente cordal con más de un vértice. Entonces, 
(a) $G$ es completo o existen dos vértices p-simpliciales/doblemente simpliciales no adyacentes.

(b) Existen dos vértices p-simpliciales/doblemente simpliciales $v_{1}$ y $v_{2}$ tales que $d\left(v_{1}, v_{2}\right)=$ $\operatorname{diam}(G)$.

Se omiten las demostraciones porque pueden obtenerse a partir de argumentos casi idénticos a algunos de los ya usados con anterioridad.

Sólo resta considerar a la clase de los grafos fuertemente cordales.

Lema 2.12. Sean $G$ un grafo y $u, v, w$ tres vértices de $G$ tales que $w$ es un máximo vecino de $v$, ecc $(w)>1$ y $2 \leq d(u, v)<\infty$. Entonces, $d(u, v)=d(u, w)+1$ y el conjunto de vértices excéntricos de $w$ es igual al conjunto de vértices excéntricos de $v$.

Demostración. Si $d(u, v)=2$, entonces $d(u, w)=1$ debido a la definición de máximo vecino. Supongamos ahora que $d(u, v)>2$. Por la desigualdad triangular, $d(u, v) \leq d(u, w)+d(w, v)$, es decir, $d(u, v) \leq d(u, w)+1$.

Sea $v v_{1} v_{2} \ldots u$ un $v u$-camino de mínima longitud. Entonces, $w v_{2} \ldots u$ es un $w u$-camino de longitud $d(u, v)-1$. Por lo tanto, $d(u, v)-1 \geq d(u, w)$, o sea, $d(u, v) \geq d(u, w)+1$. Luego, la igualdad $d(u, v)=d(u, w)+1$ es verdadera. Combinemos esto con la desigualdad $\operatorname{ecc}(w)>1$ para deducir que todo vértice excéntrico de $v$ está a una distancia mayor o igual que tres de $v$. Por consiguiente,

$$
\begin{gathered}
d(v, u)=\operatorname{ecc}(v) \Leftrightarrow d(v, u)=\max \{d(v, x): x \in V(G)\} \Leftrightarrow d(v, u)= \\
\max \{d(v, x): x \in V(G), d(v, x) \geq 3\} \Leftrightarrow d(w, u)+1= \\
\max \{d(w, x)+1: x \in V(G), d(w, x) \geq 2\} \Leftrightarrow d(w, u)=\max \{d(w, x): x \in V(G), d(w, x) \geq 2\} \\
\Leftrightarrow d(w, u)=\max \{d(w, x): x \in V(G)\} \Leftrightarrow d(w, u)=\operatorname{ecc}(w)
\end{gathered}
$$

Teorema 2.13. Sean $G$ un grafo fuertemente cordal $y v \in V(G)$. Entonces, existe un vértice excéntrico de $v$ que es simple.

Demostración. La prueba será por inducción sobre $n=|V(G)|$. El enunciado del teorema es obviamente válido para el caso de $n=1$. Supongamos ahora que siempre es verdadero cuando $n=k$, siendo $k$ un número mayor o igual que 1, y que $G$ es un grafo fuertemente cordal con $k+1$ vértices. Supongamos también que $G$ es conexo pues, en caso contrario, la demostración es trivial. La prueba se divide en casos.

Caso 1: $G$ posee un vértice universal.

Sea $w$ un vértice universal de $G$. Si $w$ es simple, entonces $G$ es completo por ser $w$ un vértice simplicial universal. Por lo tanto, la existencia de un vértice excéntrico y simple es evidente. Si $w$ no es simple y $v=w$, el hecho de que $w$ sea universal implica que cualquier vértice simple de $G$ es un vértice excéntrico de $v$.

Supongamos ahora que $v \neq w$ y que $w$ no es simple. Consideremos al grafo fuertemente cordal $G-w$. En caso de que $G-w$ no sea conexo, cualquier vértice simple de $G-w$ localizado en una componente conexa diferente de la de $v$ es un vértice simple excéntrico de $v$ en $G$. Si $G-w$ es conexo, podemos aplicar la hipótesis inductiva a este grafo para obtener un vértice $u$ que sea simple y excéntrico de $v$ en $G-w$. No es difícil ver que $u$ es simple y excéntrico de $v$ también en $G$.

Caso 2: $G$ no tiene vértices universales. 
Caso 2.a: $v$ es simple.

$\mathrm{Al}$ ser $v$ simple, podemos tomar un vértice $v^{\prime}$ que sea máximo vecino de $v$. Entonces, $v \neq v^{\prime}$ porque $v$ no es universal. Por hipótesis inductiva, existe un vértice $w$ que es simple y excéntrico de $v^{\prime}$ en $G-v$. Notar que $d\left(v^{\prime}, w\right) \geq 2$ porque, en caso contrario, $v^{\prime}$ sería un vértice universal de $G$. Como $v^{\prime}$ es un máximo vecino de $v$ en $G$ y, por ende, $N^{2}[v] \subseteq N\left[v^{\prime}\right]$, concluimos que $d(v, w) \geq 3$. Por lo tanto, las vecindades cerradas de los vértices en $N[w]$ son las mismas en $G$ y en $G-v$, lo cual implica que $w$ también es simple en $G$. Por el Lema $2.12, w$ es excéntrico de $v$ también en $G$.

Caso 2.b: $v$ no es simple y existe un vértice simple que no es adyacente a $v$.

Sea $w$ un vértice simple que no es adyacente a $v$. Si $w$ es excéntrico de $v$, no hay nada más que ver. Si no, consideremos al grafo fuertemente cordal $G-w$ que, por hipótesis inductiva, posee un vértice simple $w^{\prime}$ que es excéntrico de $v$. Entonces, a causa de la Proposición 2.1, $w^{\prime}$ es también excéntrico de $v$ en $G$, por lo que basta probar que $w^{\prime}$ es simple en $G$.

Supongamos por el contrario que $w^{\prime}$ no es simple en $G$. Veamos primero que $w \in N^{2}\left[w^{\prime}\right]$. Como $w^{\prime}$ no es simple, existen vértices $u_{1}$ y $u_{2}$ en $N\left[w^{\prime}\right]$ tales que $N\left[u_{1}\right] \nsubseteq N\left[u_{2}\right]$ y $N\left[u_{2}\right] \nsubseteq N\left[u_{1}\right]$. Si $u_{1}=w$ o $u_{2}=w$, entonces $w$ está en $N\left[w^{\prime}\right]$ y, con mayor razón, en $N^{2}\left[w^{\prime}\right]$.

Si $u_{1} \neq w$ y $u_{2} \neq w$, entonces $u_{1}$ y $u_{2}$ están en la vecindad cerrada de $w^{\prime}$ en $G-w$. Como $w^{\prime}$ es simple en $G-w$, podemos suponer sin pérdida de generalidad que $N_{G-w}\left[u_{1}\right] \subseteq N_{G-w}\left[u_{2}\right]$. Si $w \notin N_{G}\left[u_{1}\right]$, entonces $N_{G}\left[u_{1}\right] \subseteq N_{G}\left[u_{2}\right]$, contradiciendo lo que se supuso previamente. Por lo tanto, $w \in N_{G}\left[u_{1}\right]$, lo cual, sumado a que $u_{1} \in N_{G}\left[w^{\prime}\right]$, implica que $w \in N^{2}\left[w^{\prime}\right]$.

Sea $u$ un máximo vecino de $w$ en $G$. Entonces, $u \in N_{G}\left[w^{\prime}\right]$ y, por ende, $d\left(v, w^{\prime}\right) \leq d(v, u)+1$. Aplicando el Lema 2.12, se obtiene que $d\left(v, w^{\prime}\right) \leq d(v, w)$, lo cual contradice que $w$ no es un vértice excéntrico de $v$.

Luego, $w^{\prime}$ es necesariamente simple en $G$.

Caso 2.c: $v$ no es simple y es adyacente a todos los vértices simples de $G$.

Probemos que $\operatorname{diam}(G) \leq 2$. Supongamos por el contrario que $\operatorname{diam}(G) \geq 3$. Sean $x$ e $y$ vértices tales que $d(x, y)=\operatorname{diam}(G)$. Entonces, $\{x, y\} \nsubseteq N[v]$, por lo que podemos suponer sin pérdida de generalidad que $x \notin N[v]$. Como todos los vértices simples son simpliciales y adyacentes a $v$, concluimos que ninguno de ellos es adyacente a $x$. Entonces, por caso $\mathbf{2 .} \mathbf{b}, x$ posee un vértice $x^{\prime}$ simple y excéntrico, por lo que $d\left(x, x^{\prime}\right)=\operatorname{diam}(G)$. Por caso 2.a, sabemos que $x^{\prime}$ posee un vértice $x^{\prime \prime}$ simple y excéntrico, de modo que $d\left(x^{\prime}, x^{\prime \prime}\right)=\operatorname{diam}(G)$. Como $x^{\prime}$ y $x^{\prime \prime}$ son ambos adyacentes a $v$, concluimos que $d\left(x^{\prime}, x^{\prime \prime}\right) \leq 2$, lo cual es absurdo. Luego, $\operatorname{diam}(G) \leq 2$.

$\mathrm{Al}$ ser $G$ dualmente cordal, podemos aplicar el Lema 2.4 para concluir que $G$ posee un vértice universal, lo cual es una contradicción. Luego, el caso 2.c no puede ocurrir.

No quedan más casos por analizar, por lo que la demostración está completa.

\section{Corolario 2.14.}

Sea $G$ un grafo fuertemente cordal con más de un vértice. Entonces,

(a) $G$ es completo o posee dos vértices simples no adyacentes.

(b) Existen dos vértices simples $v_{1}$ y $v_{2}$ tales que $d\left(v_{1}, v_{2}\right)=\operatorname{diam}(G)$. 


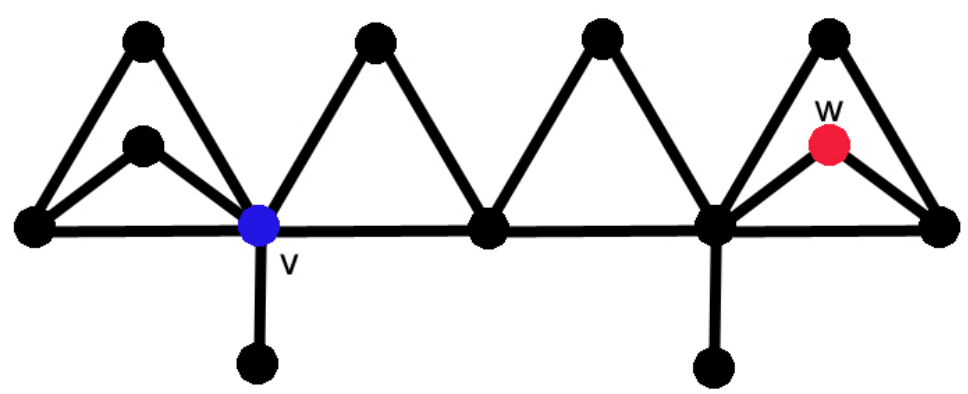

Figura 2.2: Un grafo fuertemente cordal, un vértice $v$ y un vértice simple $w$ que es excéntrico de $v$. 


\section{Capítulo 3}

\section{Separadores minimales de vértices de grafos dualmente cordales}

Hemos visto antes varias de las caracterizaciones de los grafos dualmente cordales, siendo la mayoría de ellas en términos de cliques y de vecindades. Sin embargo, no mucho se conocía acerca de los separadores de vértices de estos grafos. Por eso, uno de los propósitos de este capítulo es estudiar los separadores minimales de vértices de los grafos dualmente cordales para determinar si las propiedades que se conocen sobre cliques y vecindades tienen su correlato con otras sobre separadores minimales de vértices no conocidas hasta este momento.

El capítulo posee cuatro secciones. En la primera sección, se estudia la relación entre los árboles compatibles y los separadores minimales de vértices. En la segunda sección, se encuentran propiedades de los separadores minimales de vértices que están contenidos en vecindades cerradas. En la tercera sección, se ve cómo lo probado en la primera sección puede ser utilizado para caracterizar a los grafos dualmente cordales. Algunas de las caracterizaciones son usadas en la última sección para obtener una nueva demostración acerca de los grafos dualmente cordales hereditarios, es decir, acerca de los grafos fuertemente cordales.

Todo los grafos considerados se supondrán conexos para facilitar la escritura de las demostraciones de las propiedades que se tratarán. En todo caso, no es difícil extenderlas a grafos no conexos si son aplicadas a cada una de las componentes conexas.

\subsection{Separadores minimales de vértices y árboles compatibles}

Hemos visto que los árboles compatibles poseen muchas propiedades interesantes, lo cual ayuda a explicar por qué son tan útiles para modelar a los grafos dualmente cordales. Cuanto más sabemos acerca de ellos, más evidente se vuelve la importancia que tienen.

En esta sección, veremos que los separadores minimales de vértices se relacionan con los árboles compatibles de una manera muy similar a la que éstos lo hacen con los cliques y las vecindades. Más específicamente, el principal objetivo es demostrar que todo separador minimal de vértices induce un subárbol de todo árbol compatible.

Teorema 3.1. Sean $G$ un grafo dualmente cordal, $T$ un árbol compatible con $G, u, v$ dos vértices de $G$ y $S$ un uv-separador minimal. Entonces, $S$ induce un subárbol de $T$.

Demostración. Si $S$ consiste solamente de un vértice, entonces la demostración es trivial. En caso contrario, necesitamos probar que, todo par de vértices distintos $x$ e $y$ en $S$ satisface que $T[x, y] \subseteq S$. Si $T[x, y]=\{x, y\}$, entonces la inclusión es clara. 
Consideremos ahora el caso de que $T[x, y] \neq\{x, y\}$. Supongamos que $T[x, y]$ no es un subconjunto de $S$ y sean $z$ un vértice en $T(x, y) \backslash S, x^{\prime}$ el último vértice que precede a $z$ en $T\langle x, y\rangle$ tal que $x^{\prime} \in S$ e $y^{\prime}$ el primer vértice posterior a $z$ en $T\langle x, y\rangle$ tal que $y^{\prime} \in S$. De esta manera, $T\left(x^{\prime}, y^{\prime}\right) \cap S=\emptyset$.

Proseguimos con la demostración de una serie de hechos:

- $u \notin T\left[x^{\prime}, v\right]$ : Supongamos por el contrario que $u \in T\left[x^{\prime}, v\right]$. Sean $v_{1} v_{2} \ldots v_{n}, v_{1}=u, v_{n}=v$, un $u v$-camino tal que su único vértice en $S$ es $x^{\prime}$ e $i=\max \left\{j: u \in T\left[v_{j}, v\right]\right\}$.

Consideremos ahora al grafo $T-u$. Si $v_{i}$ y $v_{i+1}$ estuvieran en la misma componente conexa de $T-u$, entonces $u \in T\left[v_{i+1}, v\right]$ (ver Figura 3.1), lo cual contradiría la definición de $i$. Por lo tanto, $v_{i}$ y $v_{i+1}$ están en diferentes componentes conexas de $T-u$ y se infiere que $u \in T\left[v_{i}, v_{i+1}\right]$.

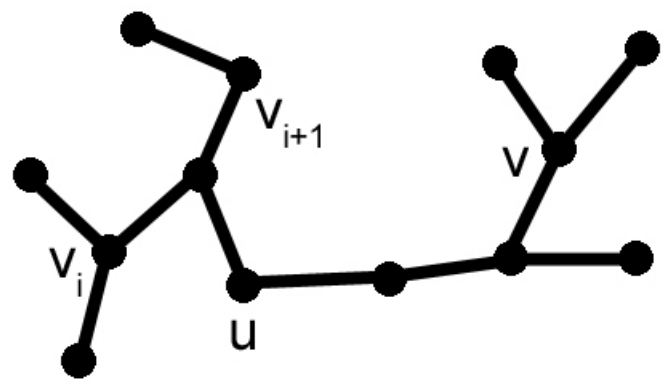

Figura 3.1: Si $u \in T\left[v_{i}, v\right]$ y $v_{i}$ y $v_{i+1}$ están en la misma componente conexa de $T-u$, entonces $u \in T\left[v_{i+1}, v\right]$.

Podemos aplicar el Teorema 1.15 para concluir que $u$ es adyacente a $v_{i+1}$. Entonces, $u v_{i+1} \ldots v$ es un $u v$-camino que, por construcción, no contiene a $x^{\prime} \mathrm{y}$, por ende, no contiene a ningún vértice de $S$, contradiciendo el hecho de que $S$ es un $u v$-separador.

Luego, $u \notin T\left[x^{\prime}, v\right]$.

- Con similar razonamiento, se demuestra que $u \notin T\left[y^{\prime}, v\right], v \notin T\left[u, x^{\prime}\right]$ y $v \notin T\left[u, y^{\prime}\right]$.

- $T\left(x^{\prime}, y^{\prime}\right)$ está contenido en la misma componente conexa de $G-S$ que $u$ : Sean $G[A]$ y $G[B]$ las componentes conexas de $G-S$ que contienen a $u$ y a $T\left(x^{\prime}, y^{\prime}\right)$, respectivamente. Consideremos el grafo $T-T\left(x^{\prime}, y^{\prime}\right)$.

Si $A$ interseca a más de una componente conexa de $T-T\left(x^{\prime}, y^{\prime}\right)$, podemos elegir dos vértices $v_{1}, v_{2} \in A$ tales que $v_{1}$ es adyacente a $v_{2}$ y que están en diferentes componentes conexas de $T-T\left(x^{\prime}, y^{\prime}\right)$. Entonces, $T\left(v_{1}, v_{2}\right) \cap T\left(x^{\prime}, y^{\prime}\right) \neq \emptyset$. Sea $w$ un vértice de esa intersección. Por el Teorema $1.15, w$ es adyacente a $v_{1}$ y a $v_{2}$, por lo que $w \in A$. Luego, $A \cap B \neq \emptyset$, lo cual implica que $A=B$.

Supongamos ahora que $A$ interseca a solamente una componente conexa de $T-T\left(x^{\prime}, y^{\prime}\right)$. Sea $D$ el conjunto de vértices de dicha componente. Como $x^{\prime}$ e $y^{\prime}$ están en diferentes componentes conexas de $T-T\left(x^{\prime}, y^{\prime}\right), x^{\prime} \notin D$ o $y^{\prime} \notin D$. Supongamos sin pérdida de generalidad que $y^{\prime} \notin D$. Ya que $S$ es un $u v$-separador minimal e $y^{\prime} \in S, y^{\prime}$ es adyacente a un vértice $w \in A$. Si $w \in T\left(x^{\prime}, y^{\prime}\right)$, entonces $A \cap B \neq \emptyset$ y por ende $A=B$. Si $w \notin T\left(x^{\prime}, y^{\prime}\right)$, entonces $w \in D$ lo cual, combinado con el hecho de que $y^{\prime} \notin D$, implica que existe un vértice $w^{\prime} \in T\left(w, y^{\prime}\right) \cap T\left(x^{\prime}, y^{\prime}\right)$. Al ser $T$ un árbol compatible, $w$ es adyacente a $w^{\prime} \mathrm{y}$, por ende, estos dos vértices están en la misma componente conexa de $G-S$. Por lo tanto, $A \cap B \neq \emptyset$, lo cual implica que $A=B$.

- Podemos demostrar con un argumento similar que $T\left(x^{\prime}, y^{\prime}\right)$ está contenido en la misma componente conexa de $G-S$ que $v$. 
Los últimos dos hechos implican que $u$ y $v$ están contenidos en la misma componente conexa de $G-S$, lo cual contradice que $u$ y $v$ estén separados por $S$.

Luego, $T[x, y] \subseteq S$. Esto es lo que necesitábamos para completar la demostración.

Una consecuencia inmediata del teorema anterior es que todo separador minimal de vértices de un grafo dualmente cordal induce un subgrafo conexo. Hay más cosas que se pueden concluir.

Proposición 3.2. [12] La familia de todos los subárboles de un árbol es Helly.

Estamos entonces en condiciones de enunciar el siguiente corolario:

Corolario 3.3. Sea $G$ un grafo dualmente cordal. Entonces, $\mathcal{S}(G)$ es Helly, su grafo de intersección es cordal y todo miembro de $\mathcal{S}(G)$ induce un subgrafo conexo de $G$.

Se probará en la tercera sección de este capítulo que el recíproco también es verdadero.

\subsection{Separadores minimales de vértices $\mathrm{y}$ vecindades}

Esta sección gira en torno a tres cuestiones, a saber, cómo y dónde pueden encontrarse separadores minimales de vértices contenidos en la vecindad cerrada de algún vértice; y cuáles son las condiciones necesarias y suficientes para que todo separador minimal de vértices de un grafo dualmente cordal esté contenido en la vecindad cerrada de un vértice, siendo esta última pregunta respondida en el Teorema 3.6.

Como forma de empezar, notemos que la caracterización de los grafos cordales dada por el Teorema 1.1 implica que, dados dos vértices no adyacentes $u \mathrm{y} v$, existe otro vértice $w$ cuya vecindad cerrada (exceptuando a $u$ y a $v$ de ser necesario) separa a $u$ y a $v$. En lo que sigue, veremos que lo mismo vale para los grafos dualmente cordales.

Teorema 3.4. Sean $u$ y $v$ dos vértices no adyacentes de un grafo dualmente cordal G. Entonces, existe un vértice $w, w \neq u$ y $w \neq v$, tal que $N[w] \backslash\{u, v\}$ es un uv-separador.

Demostración. Sea $T$ un árbol compatible con $G$. Notar que $u$ y $v$ no son adyacentes en $T$ porque no lo son en $G$, por lo que $T(u, v)$ no es vacío. Sea $w$ cualquier vértice de $T(u, v)$. Si $P$ es un $u v$-camino en $G$, entonces $P$ contiene vértices en diferentes componentes conexas de $T-w$. Esto implica que existen dos vértices $x_{1}$ y $x_{2}$ consecutivos en $P$ tales que $w \in T\left[x_{1}, x_{2}\right]$. Al ser $T$ compatible con $G,\left\{x_{1}, x_{2}\right\} \subseteq N[w]$. Como, a diferencia de $x_{1}$ y $x_{2}, u$ y $v$ no son adyacentes, $x_{1}$ o $x_{2}$ pertenece a $N[w] \backslash\{u, v\}$.

Por consiguiente, todo $u v$-camino en $G$ tiene algún vértice en $N[w] \backslash\{u, v\}$, es decir, $N[w] \backslash$ $\{u, v\}$ es un $u v$-separador de $G$.

Todo separador de vértices obviamente contiene un separador minimal de vértices lo cual, combinado con la prueba anterior, da lugar al siguiente corolario:

Corolario 3.5. Sean $G$ un grafo dualmente cordal, $T$ un árbol compatible con $G$ y u y $v$ dos vértices no adyacentes de $G$. Entonces, la vecindad cerrada de todo vértice en $T(u, v)$ contiene un uv-separador minimal. 
Más allá de lo que este corolario afirma, no resulta cierto que todo separador minimal de vértices de un grafo dualmente cordal esté contenido en la vecindad cerrada de algún vértice (ver Figura 3.2).

No obstante, esta propiedad se vuelve verdadera bajo condiciones adicionales. De hecho, obtendremos a continuación una condición necesaria y suficiente para que todo separador minimal de vértices de un grafo dualmente cordal esté contenido en alguna vecindad cerrada.

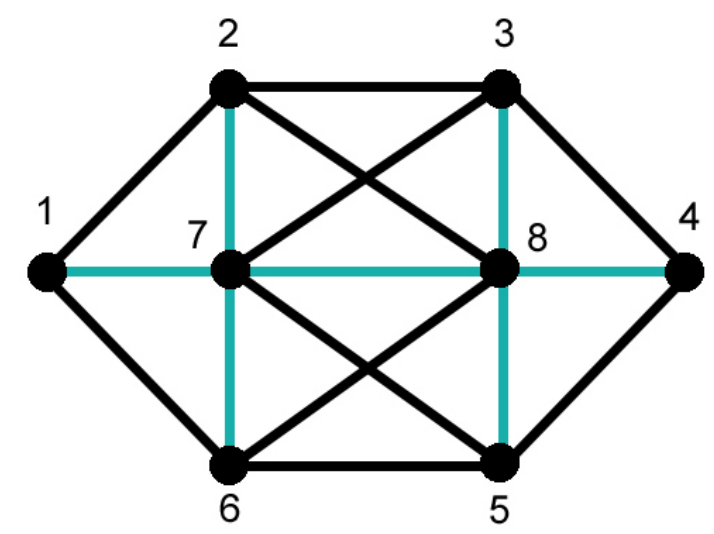

Figura 3.2: Un grafo dualmente cordal con las aristas de un árbol compatible coloreadas. $\{1,4,7,8\}$ es un 26-separador minimal, pero no está contenido en la vecindad cerrada de ningún vértice.

Teorema 3.6. Sea $G$ un grafo dualmente cordal. Entonces, todo separador minimal de vértices de $G$ está contenido en alguna vecindad cerrada si y sólo si todo ciclo sin cuerdas de longitud al menos cuatro está contenido en la vecindad cerrada de algún vértice.

Demostración. Supongamos que $G$ es un grafo dualmente cordal tal que todo separador minimal de vértices está contenido en una vecindad cerrada. Sea $C$ un ciclo sin cuerdas de $G$ de longitud al menos cuatro, si lo hay, y sean $x, y$ dos vértices no consecutivos de $C$. Como hay dos $x y$ caminos de longitud al menos dos en $C$, podemos tomar dos vértices internos $u$ y $v$ de cada uno de esos caminos. Por cada uv-camino en $G$ no completamente contenido en $G[C]$, elijamos un vértice $z \notin C$. Sean $R$ el conjunto de los vértices elegidos y $S=R \cup\{x, y\}$.

Probemos ahora que $S$ es un $u v$-separador. Sólo se necesita ver que todo $u v$-camino $P$ posee un vértice en $S$. Si $P$ no es un camino en $G[C]$, entonces tiene un vértice en $S$ por la forma en que $R$ fue construido. Además, como $C$ no posee cuerdas, hay solamente dos $u v$-caminos en $G[C]$, con $x$ e $y$ en cada uno de ellos.

Sea $S^{\prime}$ un $u v$-separador minimal contenido en $S$ y sean $P_{1}$ y $P_{2}$ los $u v$-caminos en $G[C]$ que contienen a $x$ y a $y$, respectivamente. Como $x$ es el único vértice en $S \cap V\left(P_{1}\right)$ e $y$ es el único vértice en $S \cap V\left(P_{2}\right), x$ e $y$ están en $S^{\prime}$. Por la hipótesis, $S^{\prime}$ está contenido en la vecindad cerrada de un vértice $\mathrm{y}$, por ende, $N[x] \cap N[y] \neq \emptyset$. Por lo tanto, $d(x, y)=2$. Concluimos entonces que la distancia entre cualesquiera dos vértices de $C$ es uno o dos. Por el Lema 2.4, existe un vértice $w$ tal que $C \subseteq N[w]$.

Recíprocamente, supongamos que todo ciclo sin cuerdas de longitud mayor o igual que cuatro está contenido en la vecindad cerrada de algún vértice y sea $S$ un separador minimal de dos vértices no adyacentes $a$ y $b$. La demostración de que $S$ está contenido en una vecindad cerrada puede ser realizada adaptando la prueba de que un grafo es cordal si y sólo si todo separador minimal de vértices es completo tal como aparece en [12]. 
Sean $x$ e $y$ dos vértices en $S$. Consideremos un ciclo $C$ que contenga a $x$ y a $y$ construido como en [12]. Más precisamente, se toman dos caminos $x a_{1} \ldots a_{r} y$ e $y b_{1} \ldots b_{t} x$ en los que, para todo $1 \leq i \leq r, a_{i}$ está en la misma componente conexa de $G-S$ que $a$ y, para todo $1 \leq i \leq t, b_{i}$ está en la misma componente conexa de $G-S$ que $b$, y tales que sus longitudes son lo mínimo posible. De esta manera, $C$ es el ciclo $x a_{1} \ldots a_{r} y b_{1} \ldots b_{t} x$.

Si $C$ tiene una cuerda, entonces $x y$ es dicha cuerda, por lo que $x$ e $y$ son adyacentes. Si $C$ no tiene cuerdas, entonces está contenido en la vecindad cerrada de algún vértice $w \mathrm{y}$, por ende, $x w y$ es un $x y$-camino de longitud dos.

Por lo tanto, para todo par $x, y \in S, d(x, y) \leq 2$. Por el Lema 2.4, $S$ está contenido en la vecindad cerrada de algún vértice.

Si $C$ es un ciclo de longitud cuatro o cinco, entonces dos vértices cualesquiera de él están a una distancia no mayor que dos, por lo que, por el Lema 2.4, $C$ está contenido en la vecindad cerrada de algún vértice. Se obtiene así el siguiente corolario:

Corolario 3.7. Sea $G$ un grafo dualmente cordal tal que $G$ es cordal o todo ciclo sin cuerdas de $G$ tiene longitud a lo sumo cinco. Entonces, todo separador minimal de vértices de $G$ está contenido en la vecindad cerrada de algún vértice.

Es consecuencia de esto, que si un grafo dualmente cordal posee un separador minimal de vértices no contenido en ninguna vecindad cerrada, entonces el grafo debe contener un ciclo sin cuerdas de longitud mayor o igual que seis no contenido en una vecindad cerrada. En la Figura 3.2 , ese ciclo es inducido por $\{1,2,3,4,5,6\}$.

En lo que resta de esta sección, se estudiará un caso particular de separadores minimales de vértices de grafos dualmente cordales contenidos en una vecindad cerrada, que son aquellos cuyos vértices equidistan de algún otro vértice. Resultará de mucha utilidad vincular a este asunto con la propiedad Helly.

Hasta ahora, tres familias Helly de conjuntos de vértices de un grafo dualmente cordal se han podido identificar: la familia de cliques, la familia de vecindades cerradas (y discos) y la familia de separadores minimales de vértices. El hecho de que todo miembro de estas familias induzca un subárbol de un árbol compatible fijo implica, por la Proposición 3.2, que incluso la unión de las tres familias es Helly.

Proposición 3.8. Sea $G$ un grafo dualmente cordal. Entonces, $\mathcal{C}(G) \cup \mathcal{S}(G) \cup \mathcal{D}(G)$ es una familia Helly.

Esto tiene implicaciones sobre algunas de las cuestiones vistas antes. Cuando se consideró un separador minimal de vértices $S$ contenido en la vecindad cerrada de un vértice $w$, podía ocurrir que $w \notin S$. Ahora veremos que, en los grafos dualmente cordales, $w$ puede ser siempre elegido de manera que $w \in S$.

Proposición 3.9. Sea $S$ un separador minimal de vértices de un grafo dualmente cordal tal que $S$ está contenido en la vecindad cerrada de algún vértice. Entonces, existe un vértice $w$ tal que $w \in S$ y $S \subseteq N[w]$.

Demostración. Consideremos la familia compuesta por $S$ y las vecindades cerradas de los vértices en $S$. Como $S$ está contenido en la vecindad cerrada de un vértice, esta familia es intersectante. Entonces, por Proposición 3.8, existe un vértice $w$ que es elemento de todo miembro de la familia, es decir, $w \in S$ y $w$ está en la vecindad cerrada de todo vértice de $S$. Esto último implica que $S \subseteq N[w]$. 
Dados $v$ un vértice de un grafo $G$ y un entero no negativo $i$, se define $D(v, i)=\{w \in$ $V(G): d(v, w)=i\}$. El siguiente resultado es verdadero para todo tipo de grafos:

Lema 3.10. Sean u y $v$ dos vértices no adyacentes de un grafo $G$ tales que $d(u, v)=k$. Entonces, para todo $1 \leq i<k$, existe un uv-separador minimal $S_{i}$ contenido en $D(v, i)$.

Demostración. Probaremos que, para todo $1 \leq i \leq k-1, D(v, i)$ es un uv-separador y que, por ende, contiene un $u v$-separador minimal. Veamos que todo $u v$-camino $v_{1} v_{2} \ldots v_{n}$, con $v_{1}=u \mathrm{y}$ $v_{n}=v$, posee un vértice en $D(v, i)$.

Sea $m=\min \left\{j: d\left(v_{j}, v\right) \leq i\right\}$. Entonces, $m \geq 2$. Si $d\left(v_{m}, v\right)<i$, se tiene, por la desigualdad triangular, que $d\left(v_{m-1}, v\right) \leq d\left(v_{m-1}, v_{m}\right)+d\left(v_{m}, v\right)<1+i$. Por lo tanto, $d\left(v_{m-1}, v\right) \leq i$, lo cual contradice la definición de $m$. Luego, $d\left(v_{m}, v\right)=i$, es decir, $v_{m} \in D(v, i)$.

En el caso particular de los grafos dualmente cordales, puede verse que ese tipo de separadores minimales de vértices está contenido en una vecindad cerrada.

Proposición 3.11. Sean $G$ un grafo dualmente cordal y $V^{\prime}$ un subconjunto de $V(G)$ tal que $V^{\prime}$ está contenido en la vecindad cerrada de un vértice $w$. Si todos los vértices de $V^{\prime}$ están a distancia $i$ de cierto vértice $v \notin V^{\prime}$, entonces existe un vértice $v_{i}$ tal que $d\left(v, v_{i}\right)=i-1$ y $V^{\prime} \subseteq N\left[v_{i}\right]$.

Demostración. Consideremos la familia compuesta por $N^{i-1}[v]$ y la vecindad cerrada de cada vértice de $V^{\prime}$. Como $V^{\prime} \subseteq N[w]$, esta familia es intersectante. Por la Proposición 3.8, existe un vértice $v_{i}$ que está en todo miembro de la familia. Por lo tanto, $V^{\prime} \subseteq N\left[v_{i}\right]$ y $d\left(v, v_{i}\right)=i-1$.

Proposición 3.12. Sean $G$ un grafo dualmente cordal, $T$ un árbol compatible con $G, T^{\prime}$ un subárbol de $T$ y $v \in V(G) \backslash V\left(T^{\prime}\right)$. Si todos los vértices de $T^{\prime}$ están a distancia $i$ de $v$, entonces existe un vértice $v_{i}$ tal que $d\left(v, v_{i}\right)=i-1$ y $V\left(T^{\prime}\right) \subseteq N\left[v_{i}\right]$.

Demostración. Consideremos la familia $\left\{T[v, w]: w \in V\left(T^{\prime}\right)\right\} \cup\left\{V\left(T^{\prime}\right)\right\}$. Esta familia es intersectante. Entonces, por la Proposición 3.2, podemos seleccionar un vértice $x$ que está en todos los miembros de la familia. Veamos ahora que $V\left(T^{\prime}\right) \subseteq N[x]$.

Si $V\left(T^{\prime}\right)=\{x\}$, entonces claramente $V\left(T^{\prime}\right) \subseteq N[x]$. En caso contrario, sea $w \in V\left(T^{\prime}\right)$, $w \neq x$, y consideremos un $v w$-camino $P$ en $G$ de longitud $i$. Sea $y$ el vértice que precede a $w$ en $P$, de modo que $d(v, y)=i-1$. Veamos ahora que $w$ e $y$ están en diferentes componentes conexas de $T-x$. Si no fuera así, como $x \in T[v, w], x \in T[v, y]$ también. Esto contradice que $v, y \in N^{i-1}[v]$ y que $N^{i-1}[v]$ induce un subárbol de $T$.

Podemos inferir entonces que $x \in T[w, y]$. Como $T$ es compatible con $G$, concluimos que $w$ es adyacente a $x$

De esta manera, la inclusión $V\left(T^{\prime}\right) \subseteq N[x]$ queda de manifiesto. Podemos aplicar ahora la Proposición 3.11 para deducir la existencia de un vértice $v_{i}$ tal que $V\left(T^{\prime}\right) \subseteq N\left[v_{i}\right]$ y $d\left(v, v_{i}\right)=$ $i-1$.

Corolario 3.13. Sean $G$ un grafo dualmente cordal y $u$ y $v$ dos vértices no adyacentes tales que $d(u, v)=k$. Si $S_{i}$ es un uv-separador minimal contenido en $D(v, i)$, para algún $1 \leq i<k$, entonces existe un vértice $v_{i}$ tal que $d\left(v, v_{i}\right)=i-1$ y $S_{i} \subseteq N\left[v_{i}\right]$.

Demostración. Al ser $G$ dualmente cordal, podemos tomar un árbol $T$ compatible con $G$. Por el Teorema 3.1, $T\left[S_{i}\right]$ es un subárbol de $T$. Entonces, la Proposición 3.12 aplicada a $T\left[S_{i}\right]$ nos da el vértice $v_{i}$ que se buscaba.

Por consiguiente, se puede probar la siguiente propiedad: 
Proposición 3.14. Sean $G$ un grafo dualmente cordal $y u, v$ dos vértices no adyacentes de $G$ tales que $d(u, v)=k$. Entonces, existe un camino $v_{1} \ldots v_{k} u, v=v_{1}, y k-1$ uv-separadores minimales disjuntos $S_{1}, \ldots, S_{k-1}$ tales que, para todo $1 \leq i \leq k-1, S_{i} \subseteq N\left[v_{i}\right]$.

Demostración. Para cada $i, 1 \leq i \leq k-1$, sea $S_{i}$ un $u v$-separador minimal contenido en $D(v, i)$. Podemos aplicar el Corolario 3.13 para obtener un vértice $v_{i}$ tal que $S_{i} \subseteq N\left[v_{i}\right]$ y $d\left(v, v_{i}\right)=i-1$.

Sea ahora $v_{k}$ cualquier vértice adyacente a $u$ y a distancia $k-1$ de $v$. Veamos que $v_{1} \ldots v_{k} u$ es el camino deseado. Por construcción, $d\left(v, v_{1}\right)=0$, y así $v_{1}=v$. Queda demostrar que, para todo $1 \leq i \leq k-1, v_{i}$ es adyacente a $v_{i+1}$. Lo probaremos primero para $1 \leq i \leq k-2$. Sea $Q$ un $u v$-camino de longitud mínima y sea $z$ el vértice de $Q$ a distancia $i+1$ de $v$ y a distancia $k-i-1$ de $u$. Como $z$ es el único vértice de $Q$ a distancia $i+1$ de $v$, concluimos que $z$ es necesariamente un elemento de $S_{i+1}$. Entonces, $v_{i+1}$ es adyacente a $z$ y, por la desigualdad triangular, $d\left(v_{i+1}, u\right) \leq d\left(v_{i+1}, z\right)+d(z, u) \leq k-i$. Recordemos también que $d\left(v_{i+1}, v\right)=i$, por lo que $v_{i+1}$ puede ser encontrado en un $u v$-camino de longitud mínima. Por consiguiente, $v_{i+1}$ está necesariamente en $S_{i}$. Al ser $v_{i}$ adyacente a todos los vértices de $S_{i}$, concluimos que $v_{i}$ es adyacente a $v_{i+1}$.

Para $i=k-1$, es una consecuencia inmediata de nuestra elección que $v_{k} \in S_{k-1}$. Por lo tanto, $v_{k-1}$ es adyacente a $v_{k}$. Esto concluye la demostración.

\subsection{Caracterizaciones}

Recordemos que ya se ha probado que cada separador minimal de vértices de un grafo dualmente cordal $G$ induce un subárbol de todo árbol compatible con $G$ y, por ende, induce un subgrafo conexo. También se ha visto que la familia de separadores minimales de vértices es Helly y que su grafo de intersección es cordal. A la hora de considerar a los cliques, aparecen propiedades que pueden ser enunciadas en términos similares: que todo clique induce un subárbol de todo árbol compatible, por un lado; y que $\mathcal{C}(G)$ es Helly y $K(G)$ es cordal, por el otro. Dado que estas dos propiedades pueden ser usadas para caracterizar a los grafos dualmente cordales, es de nuestro interés saber si las propiedades sobre separadores minimales de vértices también conducen a caracterizaciones. Afortunadamente, esto es cierto y esta sección está dedicada a probar las caracterizaciones.

Teorema 3.15. Un grafo $G$ es dualmente cordal si y sólo si posee un árbol generador $T$ tal que todo separador minimal de vértices de $G$ induce un subárbol de $T$.

Demostración. Por el Teorema 3.1, resta probar una de las implicaciones.

Supongamos que $T$ es un árbol generador como el que se describe arriba. Sean $x$ e $y$ dos vértices adyacentes de $G$ y sea $z \in T(x, y)$. Supongamos que $x$ y $z$ no son adyacentes. Sea $S$ un $x z$-separador minimal. Como $S$ induce un subárbol de $T$ y debe contener un vértice en $T[x, z], T\langle y, z\rangle$ es un $y z$-camino en $G-S$ (ver Figura 3.3). Por lo tanto, y y $z$ están en la misma componente conexa de $G-S$. Pero $x$ también está en esa componente por ser adyacente a $y$, contradiciendo que $x$ y $z$ están separados por $S$.

Entonces, $x$ y $z$ son necesariamente adyacentes. Similarmente, podemos probar que $y$ es adyacente a $z$. Luego, por el Teorema $1.15, G$ es dualmente cordal.

Si se analizan los pasos de esta demostración, queda claro que no es necesario saber que todo separador minimal de vértices de $G$ induce un subárbol de $T$ para concluir que $G$ es dualmente cordal. En realidad, la demostración requiere que, por cada dos vértices no adyacentes $u$ y $v$, exista al menos un $u v$-separador minimal que induzca un subárbol de $T$. Esto conduce a una caracterización ligeramente diferente de los grafos dualmente cordales. 


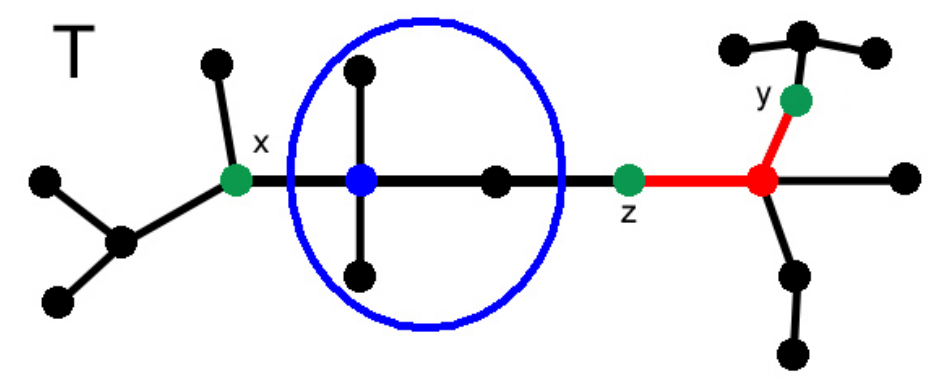

Figura 3.3: Como $S$ induce un subárbol y contiene un vértice en $T[x, z]$ (supongamos que es el azul), los vértices de $S$ están contenidos en el interior del círculo. Por lo tanto, $T\langle y, z\rangle$ es un yz-camino en $G-S$.

Teorema 3.16. Sean $G$ un grafo y $\mathcal{F}$ una familia de separadores minimales de vértices de $G$ de modo que, por cada dos vértices no adyacentes u y $v, \mathcal{F}$ contiene un uv-separador. Entonces, $G$ es dualmente cordal si y sólo si $G$ posee un árbol generador $T$ tal que todo miembro de $\mathcal{F}$ induce un subárbol de $T$.

La demostración del Teorema 3.16 es casi idéntica a la del Teorema 3.15. La única diferencia es que se requiere que $S$ esté en $\mathcal{F}$.

Ahora se presenta la segunda caracterización principal:

Teorema 3.17. Un grafo $G$ es dualmente cordal si y sólo si todo separador minimal de vértices de $G$ induce un subgrafo conexo, $\mathcal{S}(G)$ es Helly y el grafo de intersección de $\mathcal{S}(G)$ es cordal.

Demostración. Por el Corolario 3.3, nos resta probar una de las implicaciones.

Supongamos que todo separador minimal de vértices induce un subgrafo conexo, que la familia de separadores minimales de vértices es Helly y que su grafo de intersección es cordal. Las últimas dos condiciones implican que existe un árbol $T$ cuyo conjunto de vértices es $V(G)$ y tal que todo separador minimal de vértices de $G$ induce un subárbol de $T$ [23]. Tomemos a $T$ de modo que $s(T):=\sum_{v w \in E(T)} d_{G}(v, w)$ sea mínima. Veamos que $T$ es un árbol generador de $G$. Si no lo fuera, sean $v$ y $w$ dos vértices adyacentes en $T$ pero no en $G$, siendo $d(v, w)=k$. Consideremos a la familia $\mathcal{F}$ formada por todos los separadores minimales de vértices que contienen tanto a $v$ como a $w$, en caso de que los hubiera, un $v w$-separador minimal $S_{1}$ contenido en $N[v]$ y un $v w$-separador minimal $S_{2}$ contenido en $D(w, k-1)$, cuya existencia está asegurada por el Lema 3.10. Se probará ahora que $\mathcal{F}$ es intersectante.

Sea $S$ un separador minimal de vértices que contiene a $v$ y a $w$. Al inducir $S$, por hipótesis, un subgrafo conexo, existe un $v w$-camino $P$ cuyos vértices están todos en $S$. Como $S_{i}, i=1,2$, separa a $v$ y a $w$, debe contener un vértice de $P$. Luego, $S \cap S_{i} \neq \emptyset$.

Sea $P$ ahora un $v w$-camino de longitud mínima. Entonces, $P$ tiene un vértice $x$ tal que $d(v, x)=1, d(w, x)=k-1$ y ningún otro vértice de $P$ satisface alguna de esas igualdades. Por consiguiente, $x \in S_{1} \cap S_{2}$. Luego, $\mathcal{F}$ es intersectante.

Como $\mathcal{F}$ es intersectante y $\mathcal{S}(G)$ es Helly, existe un vértice $u$ que está en todo miembro de $\mathcal{F}$. Sean $T[A]$ y $T[B]$ las componentes conexas de $T-v w$ que contienen a $v$ y a $w$, respectivamente. Si $u \in A$, sea $T^{\prime}=T-v w+u w$. Veamos ahora que todo separador minimal de vértices de $G$ induce un subárbol de $T^{\prime}$ (ver Figura 3.4). Sea $S$ un separador minimal de vértices de $G$. Si $S \subseteq A$ o $S \subseteq B$, entonces $S$ induce el mismo subárbol en $T$ y en $T^{\prime}$. 
Caso contrario, se tienen dos vértices $y, z \in S$ tales que $y \in A$ y $z \in B$. Como $S$ induce un subárbol de $T$ y $v, w \in T[y, z]$, se concluye que $v, w \in S$ y, por ende, $u \in S$ también. Entonces, $v$ y $w$ están conectados en $T^{\prime}$ por el camino que se forma al encadenar el camino $T\langle v, u\rangle=T^{\prime}\langle v, u\rangle$ y la rista $u w$. Los vértices de este camino están contenidos en $S$ porque $u, v \in S$ y $T[S]$ es conexo. Además, los vértices de todo otro par de elementos de $S$ que son adyacentes en $T$ son aún adyacentes en $T^{\prime}$. Esto es suficiente para concluir que $S$ induce un subárbol de $T^{\prime}$.
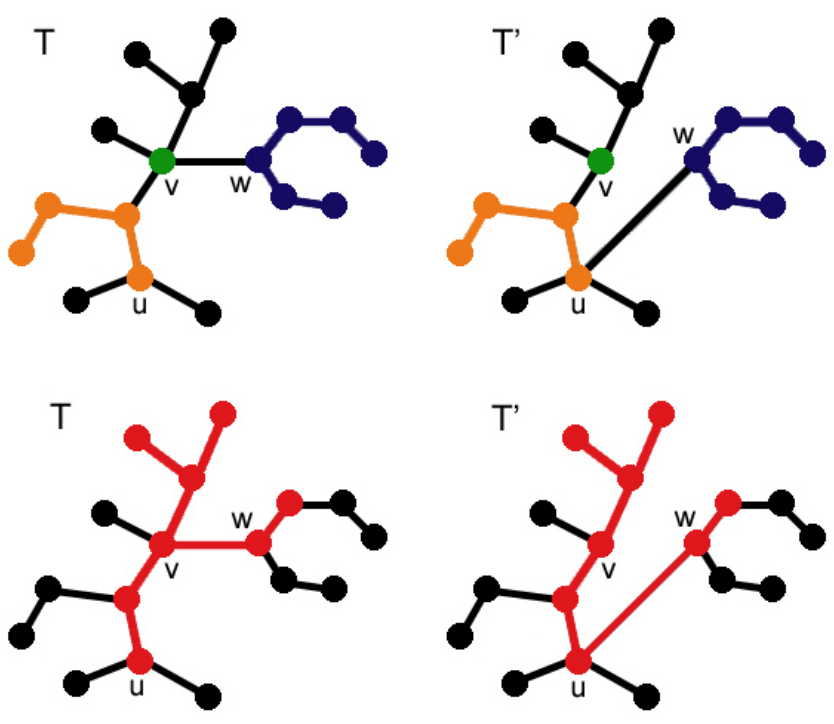

Figura 3.4: Si todo separador minimal de vértices induce un subárbol de $T$, entonces todo separador minimal de vértices inducirá un subárbol de $T^{\prime}$ también. Se da aquí una idea gráfica de eso para los casos en que el separador está contenido en $A$ o en $B$ (arriba) o que tenga vértices de ambos conjuntos (abajo).

Sin embargo, $s\left(T^{\prime}\right)<s(T)$ porque $d(u, w)=k-1$ y $d(v, w)=k$, lo cual contradice la elección de $T$. Si $u \in B$, entonces podemos remover la arista $v w$ y agregar $u v$ a $T$ y surgirá una contradicción similar. Luego, $T$ es necesariamente un árbol generador de $G$ y, por el Teorema $3.15, G$ es dualmente cordal.
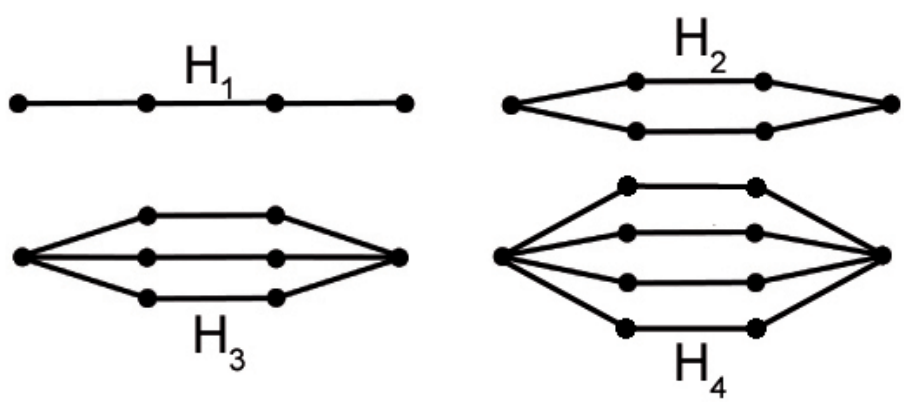

Figura 3.5: Los primeros grafos de una familia $\left\{H_{n}\right\}_{n \geq 1}$ para la cual el número de separadores minimales de vértices crece exponencialmente. 
Notar que esta caracterización no nos permite decidir de un modo eficiente si un grafo es dualmente cordal o no. Se necesita, para testear las tres condiciones de la caracterización, una lista de todos los separadores minimales del grafo. Sin embargo, podría haber un número exponencial de separadores minimales de vértices.

Consideremos, por ejemplo, la familia de grafos que se muestra en la Figura 3.5. El grafo $H_{n}$ está compuesto por $n$ caminos de longitud dos que conectan a dos vértices $u$ y $v$ y que no tienen vértices internos en común. Como todo $u v$-separador minimal de $H_{n}$ debe contener un vértice de cada camino, concluimos que $H_{n}$ posee $2^{n} u v$-separadores minimales. Si agregamos un vértice universal a $H_{n}$, el grafo resultante es dualmente cordal y también posee $2^{n} u v$-separadores minimales.

No obstante, se puede obtener una caracterización de los grafos dualmente cordales que dependa de una familia de separadores minimales de vértices cuyo número de miembros no exceda a $2|E(\bar{G})|$. En vista del Teorema 3.16 y los pasos de la demostración del Teorema 3.17, se puede probar lo siguiente:

Teorema 3.18. Sean $G$ un grafo y $\mathcal{F}$ una familia de separadores minimales de vértices de $G$ tal que, por cada dos vértices no adyacentes $v$ y $w$ de $G$, existen dos vw-separadores minimales $S_{1}$ y $S_{2}$ en $\mathcal{F}$ (pueden ser iguales) tales que $S_{1} \cap S_{2} \neq \emptyset$ y $S_{1} \cap S_{2} \subseteq N^{k-1}[v] \cap N^{k-1}[w]$, siendo $k$ la distancia de $v$ a $w$ en $G$. Entonces, $G$ es dualmente cordal si y sólo si todo miembro de $\mathcal{F}$ induce un subgrafo conexo de $G, \mathcal{F}$ es Helly y el grafo de intersección de $\mathcal{F}$ es cordal.

Observar que, para construir una familia $\mathcal{F}$ como la del teorema, una de las formas posibles es definir a $S_{1}$ y a $S_{2}$ como se hizo en la prueba del Teorema 3.17. El Teorema 3.16 nos permite considerar solamente a los miembros de $\mathcal{F}$ en lugar de todos los separadores minimales de vértices de $G$ (si los hubiera más). La condición de que $S_{1} \cap S_{2} \neq \emptyset$ garantiza que $\mathcal{F}$, definida de un modo similar al de el Teorema 3.17, sea intersectante. La condición $S_{1} \cap S_{2} \subseteq N^{k-1}[v] \cap N^{k-1}[w]$ nos permite concluir nuevamente que $s\left(T^{\prime}\right)<s(T)$.

\subsection{Separadores minimales de vértices $\mathrm{y}$ grafos fuertemente cordales}

Se presenta a esta sección como una aplicación de la anterior.

Un análisis de las condiciones del Teorema 3.17 para que un grafo sea dualmente cordal revela que ninguna de ellas puede ser omitida para simplificar la caracterización.

$C_{4}$, el ciclo de cuatro vértices, satisface que $\mathcal{S}\left(C_{4}\right)$ es Helly y $L\left(\mathcal{S}\left(C_{4}\right)\right)$ es cordal, pero los separadores minimales de vértices de $C_{4}$ o de cualquier otro ciclo de mayor longitud no inducen subgrafos conexos.

También sabemos que los soles no son grafos dualmente cordales. Todo separador minimal de vértices de un sol induce un subgrafo conexo. $L(\mathcal{S}(3-s o l))$ es cordal, pero $\mathcal{S}(3-s o l)$ no es Helly. Por otro lado, para todo $k \geq 4, \mathcal{S}(k-s o l)$ es Helly, pero $L(\mathcal{S}(k-s o l))$ no es cordal.

Todos estos grafos son exactamente los subgrafos inducidos prohibidos minimales de los grafos fuertemente cordales. El objetivo de esta sección es probar esto último de una manera diferente, a través del uso de los separadores minimales de vértices y de las caracterizaciones vistas en la sección anterior.

La demostración se dividirá en dos proposiciones, inspiradas en las Proposiciones 1.18 y 1.19, y el teorema principal al final.

Lema 3.19. Sea $S$ un separador minimal de vértices de un grafo cordal $G$ y $v \notin S$. Entonces, existe un vértice $w$ tal que $S \subseteq N[w]$ y $v$ y $w$ están en diferentes componentes conexas de $G-S$. 
Demostración. Como $S$ es un separador minimal de vértices de un grafo cordal, existen cliques $C_{1}$ y $C_{2}$ en $G$ tales que $C_{1} \cap C_{2}=S$ y $C_{1} \backslash C_{2}$ y $C_{2} \backslash C_{1}$ están contenidos en componentes conexas distintas de $G-S$ [16]. Podemos suponer sin pérdida de generalidad que $C_{1} \backslash C_{2}$ y $v$ no están contenidos en la misma componente conexa de $G-S$. Entonces, cualquier vértice $w \in C_{1} \backslash C_{2}$ satisface la condición requerida.

Lema 3.20. [2] Una familia $\mathcal{F}$ es Helly si y sólo si, para toda tripla $u, v, w$ de elementos de $\mathcal{F}$, los miembros de la subfamilia $\mathcal{F}_{\text {uvw }}$ de miembros de $\mathcal{F}$ que contienen al menos dos elementos de la tripla tienen intersección no vacía.

Proposición 3.21. Sea $G$ un grafo cordal tal que $\mathcal{S}(G)$ no es Helly. Entonces, el 3-sol es un subgrafo inducido de $G$.

Demostración. Como $\mathcal{S}(G)$ no es Helly, tomemos $v_{1}, v_{2}, v_{3} \in V(G)$ tales que los miembros de $\mathcal{S}(G)$ que contienen al menos dos elementos de $\left\{v_{1}, v_{2}, v_{3}\right\}$ tienen intersección vacía. Entonces, existen $S_{1}, S_{2}, S_{3} \in \mathcal{S}(G)$ tales que $S_{1} \cap\left\{v_{1}, v_{2}, v_{3}\right\}=\left\{v_{2}, v_{3}\right\}, S_{2} \cap\left\{v_{1}, v_{2}, v_{3}\right\}=\left\{v_{1}, v_{3}\right\}$ y $S_{3} \cap\left\{v_{1}, v_{2}, v_{3}\right\}=\left\{v_{1}, v_{2}\right\}$.

Por el Lema 3.19, podemos elegir, para $i=1,2,3$, un vértice $w_{i}$ tal que $S_{i} \subseteq N\left[w_{i}\right]$ y $v_{i}$ y $w_{i}$ están en diferentes componentes conexas de $G-S_{i}$.

$\mathrm{Al}$ ser los separadores minimales de vértices de $G$ completos, queda claro que $\left\{v_{1}, v_{2}, v_{3}\right\}$ es completo. También, debido a la forma en que fueron elegidos, $N\left[w_{1}\right] \cap\left\{v_{1}, v_{2}, v_{3}\right\}=\left\{v_{2}, v_{3}\right\}$, $N\left[w_{2}\right] \cap\left\{v_{1}, v_{2}, v_{3}\right\}=\left\{v_{1}, v_{3}\right\}$ y $N\left[w_{3}\right] \cap\left\{v_{1}, v_{2}, v_{3}\right\}=\left\{v_{1}, v_{2}\right\}$.

Supongamos ahora que $1 \leq i, j \leq 3$ y que $i \neq j$. Entonces, $w_{j} \notin S_{i}$ porque $v_{j} \in S_{i}$ y $w_{j}$ no es adyacente a él. Como $w_{j}$ es adyacente a $v_{i}$, podemos concluir que $w_{i}$ y $w_{j}$ están en diferentes componentes conexas de $G-S_{i}$. Por lo tanto, $w_{i}$ y $w_{j}$ no son adyacentes.

Luego, $\left\{v_{1}, v_{2} . v_{3}, w_{1}, w_{2}, w_{3}\right\}$ induce un 3-sol en $G$.

Proposición 3.22. Sea $G$ un grafo cordal tal que $\mathcal{S}(G)$ es Helly y $L(\mathcal{S}(G))$ no es cordal. Entonces, existe un número $k, k \geq 4$, tal que $G$ tiene un $k$-sol inducido.

Demostración. Sea $R$ un ciclo sin cuerdas de longitud mínima en $L(\mathcal{S}(G))$, con sucesión de vértices $S_{1} S_{2} \ldots S_{k} S_{1}, k \geq 4$. Sean $v_{i} \in S_{i} \cap S_{i+1}, i=1, \ldots, k-1$, y $v_{k} \in S_{1} \cap S_{k}$. Como $R$ no tiene cuerdas, todos estos vértices son distintos y forman un ciclo $C$ en $G$.

Si los vértices de $C$ forman un conjunto completo, podemos tomar, por el Lema 3.19, un vértice $w_{1}$ tal que $S_{1} \subseteq N\left[w_{1}\right]$ y tal que $w_{1}$ y $\left\{v_{1}, v_{2}, \ldots, v_{k}\right\} \backslash\left\{v_{1}, v_{k}\right\}$ están contenidos en componentes conexas distintas de $G-S_{1}$. Similarmente, para $i=2, \ldots, k$, sea $w_{i}$ un vértice tal que $S_{i} \subseteq N\left[w_{i}\right]$, y $w_{i}$ y $\left\{v_{1}, v_{2}, \ldots, v_{k}\right\} \backslash\left\{v_{i-1}, v_{i}\right\}$ están contenidos en diferentes componentes conexas de $G-S_{i}$.

Consideremos dos números $1 \leq i, j \leq k, i \neq j$. No es difícil ver que $w_{j} \notin S_{i}$ y que $w_{i}$ y $w_{j}$ están en diferentes componentes conexas de $G-S_{i}$, por lo que $w_{i}$ no es adyacente a $w_{j}$. Por lo tanto, $\left\{v_{1}, \ldots, v_{k}, w_{1}, \ldots, w_{k}\right\}$ induce un $k$-sol.

Si los vértices de $C$ no forman un conjunto completo, sean $v_{i}, v_{j}$ en $C$ tales que $v_{i}$ y $v_{j}$ no son adyacentes y $S$ un $v_{i} v_{j}$-separador minimal. Como hay dos $v_{i} v_{j}$-caminos en $C, S$ debe contener dos vértices no consecutivos de $C$, sean ellos $v_{l}$ y $v_{m}$. Sin pérdida de generalidad, supongamos que $l<m$. Sea $A=\left\{S_{n}: l+1 \leq n \leq m\right\}$. Si $|A| \leq 2$, entonces es claro que la intersección entre $S$ y todo elemento de $A$ es no vacía. Si $|A| \geq 3$, sea $R^{\prime}$ el ciclo $S S_{l+1} S_{l+2} \ldots S_{m} S$. Al ser $R^{\prime}$ de menor longitud que $R, R^{\prime}$ posee una cuerda. Como $R$ no tiene cuerdas, $S$ debe ser uno de los extremos de la cuerda. La adición de esta cuerda a $R^{\prime}$ genera dos nuevos ciclos. Si alguno de ellos tiene longitud mayor o igual que cuatro, entonces tiene una cuerda, y nuevamente $S$ debe 
ser uno de sus extremos. Se continúa este procedimiento hasta concluir que la intersección entre $S$ y todo elemento de $A$ es no vacía. Similarmente, si se define $B=\left\{S_{1}, S_{2}, \ldots, S_{k}\right\} \backslash A$, también se puede deducir que la intersección entre $S$ y todo elemento de $B$ es no vacía.

Ya que $\mathcal{S}(G)$ es Helly, podemos tomar $u_{i} \in S_{i} \cap S_{i+1} \cap S, i=1, \ldots, k-1$, y $u_{k} \in S_{1} \cap S_{k} \cap S$. Entonces, $\left\{u_{1}, u_{2}, \ldots, u_{k}\right\}$ es completo por ser un subconjunto de $S$. Se puede encontrar ahora un $k$-sol inducido como se hizo en el caso en que los vértices de $C$ eran adyacentes de a pares.

Teorema 3.23. Sea $G$ un grafo. Entonces, $G$ es dualmente cordal hereditario si y sólo si $G$ es cordal y sin soles inducidos.

Demostración. Como los ciclos de longitud mayor o igual que cuatro y los soles no son grafos dualmente cordales, ninguno de ellos es un subgrafo inducido de un grafo dualmente cordal hereditario.

Supongamos ahora que $G$ es cordal y sin soles inducidos. Entonces, todo separador minimal de vértices de $G$ es completo, por lo que claramente induce un subgrafo conexo. Por las Proposiciones 3.21 y $3.22, \mathcal{S}(G)$ es Helly y $L(\mathcal{S}(G))$ es cordal. Luego, por el Teorema $3.17, G$ es dualmente cordal.

Todo subgrafo inducido de $G$ es también cordal y sin soles inducidos, y por ende dualmente cordal. Por lo tanto, $G$ es un grafo dualmente cordal hereditario. 


\section{Capítulo 4}

\section{Grafos básicamente cordales}

Sea $G$ un grafo cordal conexo. Sabemos que $G$ tiene por lo menos un árbol clique. El conjunto de vértices de cada árbol clique es $\mathcal{C}(G)$. Además, $K(G)$ es dualmente cordal y el conjunto de vértices de cada árbol compatible con $K(G)$ es $V(K(G))$, que es igual a $\mathcal{C}(G)$.

Dado que los árboles clique de $G$ y los árboles compatibles de $K(G)$ tienen el mismo conjunto de vértices, es posible compararlos, acción que revelará cuán relacionados están. Como en el capítulo anterior, se supondrá que los grafos estudiados son siempre conexos.

Proposición 4.1. Sea $G$ un grafo cordal. Entonces, todo árbol clique de $G$ es compatible con $K(G)$.

Demostración. Sean $T$ un árbol clique de $G$ y $C$ un clique de $G$. La vecindad cerrada de $C$ en $K(G)$ es igual a $\bigcup_{v \in C} \mathcal{C}_{v}$, conjunto que induce un subárbol al ser $T$ un árbol clique. Luego, $T$ es compatible con $K(G)$.

Sin embargo, no es cierto que, para todo grafo cordal $G$, todo árbol compatible con $K(G)$ es un árbol clique de $G$. Por ejemplo, consideremos el grafo $G$ de la Figura 1.5. El árbol compatible con $K(G)$ que se muestra ahí no es un árbol clique de $G$ porque $\mathcal{C}_{d}$ no induce un subárbol (ver Figura 4.1).

Se dirá que un grafo es básicamente cordal si es cordal y sus árboles clique son exactamente los árboles compatibles de su grafo clique.

Uno de los principales objetivos de este capítulo es el desarrollo de herramientas para responder tan fácilmente como sea posible si un grafo cordal $G$ dado es básicamente cordal o no, siendo la principal caracterización lograda de los grafos básicamente cordales la del Teorema 4.26. Debido a la Proposición 4.1, el problema se reduce a determinar si todo árbol compatible con $K(G)$ es un árbol clique de $G$.

Para proseguir, son necesarias propiedades adicionales acerca de los grafos cordales, los árboles clique y los árboles compatibles.

En primer lugar, notemos que se puede hallar un árbol clique para un grafo cordal dado en tiempo polinomial usando algoritmos numéricos. El más clásico de ellos radica en la siguiente caracterización:

Teorema 4.2. [18] Sean $G$ un grafo y $K(G)^{w}$ el grafo obtenido a partir de $K(G)$ dándole a cada arista $C C^{\prime}$ el peso $\left|C \cap C^{\prime}\right|$. Entonces, $T$ es un árbol clique de $G$ si y sólo si $T$ es un árbol generador de $K(G)^{w}$ de peso máximo, siendo el peso total igual a $\sum_{C \in \mathcal{C}(G)}|C|-|V(G)|$. 

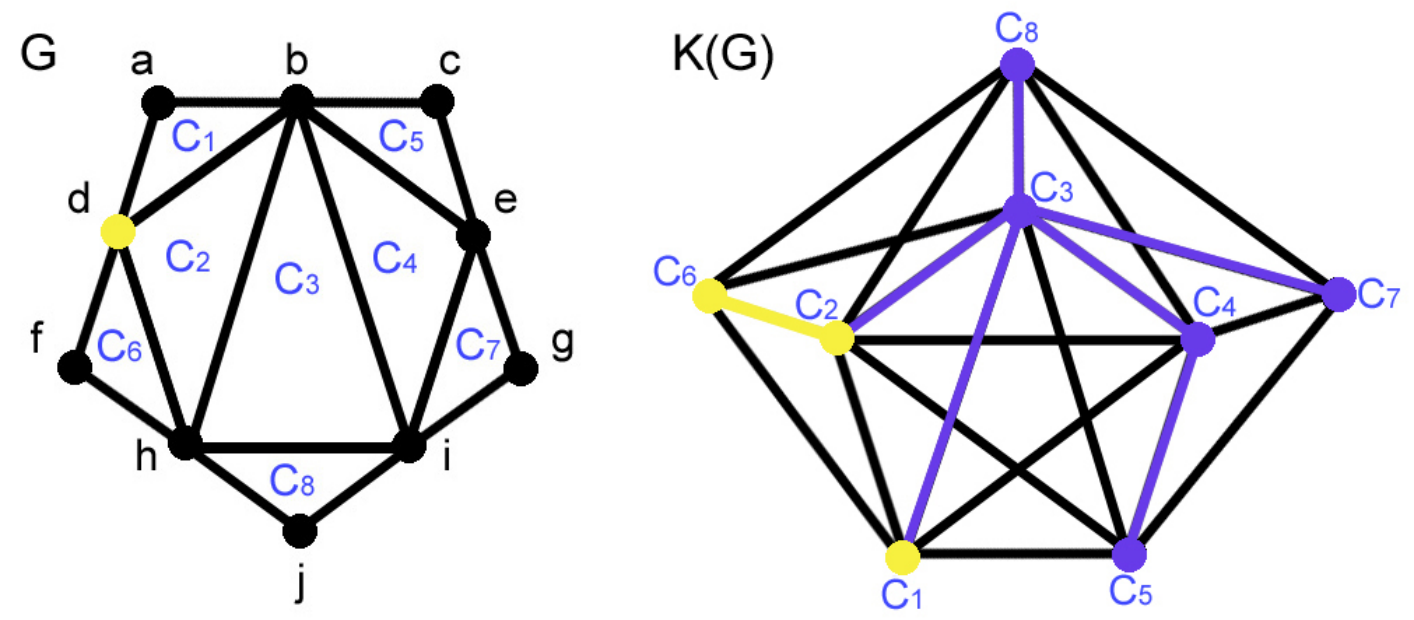

Figura 4.1: Un grafo cordal $G$, su grafo clique y un árbol compatible con $K(G)$ que no es un árbol clique de $G$ porque $\mathcal{C}_{d}$ no induce un subárbol.

Afortunadamente, existe un procedimiento similar para obtener un árbol compatible de un grafo dualmente cordal.

Teorema 4.3. [5] Sean $G$ un grafo y $G^{w}$ el mismo grafo con cada arista uv teniendo peso $p(u, v)=|N[u] \cap N[v]|$. Entonces, un árbol $T$ es compatible con $G$ si y sólo si $T$ es un árbol generador de peso máximo de $G^{w}$ con peso total igual a $2|E(G)|$.

Demostración. Sea $T$ un árbol generador de $G^{w}$. Entonces:

$$
\begin{gathered}
\sum_{u v \in E(T)} p(u, v)=\sum_{u v \in E(T)}|N[u] \cap N[v]|=\sum_{u v \in E(T)} \sum_{w \in V(G)}|\{w\} \cap N[u] \cap N[v]|= \\
\sum_{w \in V(G)} \sum_{u v \in E(T)}|\{w\} \cap N[u] \cap N[v]|=\sum_{w \in V(G)}|E(T[N[w]])| \leq \sum_{w \in V(G)}(|N[w]|-1)= \\
\sum_{w \in V(G)}(1+\operatorname{deg}(w)-1)=\sum_{w \in V(G)} \operatorname{deg}(w)=2|E(G)|
\end{gathered}
$$

La igualdad vale si y sólo si, para todo $w \in V(G),|E(T[N[w]])|=|N[w]|-1$, es decir, si y sólo si la vecindad cerrada de todo vértice de $G$ induce un subárbol de $T$.

Las tres propiedades a continuación serán acerca de separadores minimales de vértices, los cuales nos darán un mejor conocimiento de las aristas de los árboles clique. Mientras que los separadores minimales de vértices de los grafos dualmente cordales fueron el tema del capítulo anterior, esta vez serán los separadores minimales de vértices de los grafos cordales los que contarán con nuestra atención.

Como los separadores minimales de vértices de los grafos cordales son completos, es a priori posible que algunos de ellos sean cliques. El próximo resultado mostrará que ese no es el caso.

Dado un grafo $G$, dos de sus cliques $C_{1}$ y $C_{2}$ forman un par separador si $C_{1} \cap C_{2}$ separa a todo par de vértices tal que uno de ellos está en $C_{1} \backslash C_{2}$ y el otro está en $C_{2} \backslash C_{1}$. Esta definición implica que $C_{1} \cap C_{2}$ es un separador minimal de vértices. Es incluso cierto que todo separador minimal de vértices de un grafo cordal puede ser expresado de esa manera: 
Teorema 4.4. [16] Sean $G$ un grafo cordal y $S \in \mathcal{S}(G)$. Entonces, existe un par separador $C_{1}$, $C_{2}$ tal que $S=C_{1} \cap C_{2}$.

La importancia de los pares separadores radica en la relación que existe entre ellos y los árboles clique. Si nos preguntamos qué aristas pueden aparecer en un árbol clique, el siguiente teorema nos da la respuesta:

Teorema 4.5. [16] Sean $C_{1}$ y $C_{2}$ dos cliques distintos de un grafo cordal $G$. Entonces, existe un árbol clique $T$ de $G$ tal que $C_{1} C_{2} \in E(T)$ si y sólo si $C_{1}$ y $C_{2}$ forman un par separador.

Finalmente, es interesante notar que, si se conoce sólo un árbol clique de cierto grafo cordal, es posible determinar cuáles pueden ser las aristas de los otros árboles clique, si los hubiera.

Teorema 4.6. [16] Sean $G$ un grafo cordal, $T$ un árbol clique de $G$ y $C_{1}, C_{2} \in \mathcal{C}(G), C_{1} \neq C_{2}$. Entonces, existe un árbol clique de $G$ que tiene a $C_{1} C_{2}$ como arista si y sólo si existen dos cliques de $G$ adyacentes en $T\left\langle C_{1}, C_{2}\right\rangle$ y cuya intersección es igual a $C_{1} \cap C_{2}$.

Ahora estamos muy cerca de establecer una condición necesaria y suficiente para que un grafo cordal no sea básicamente cordal. Como paso previo, se consigna un resultado que es posible probar usando argumentos similares a aquellos que pueden ser utilizados para demostrar la efectividad de algunos algoritmos como el de Kruskal [17].

Proposición 4.7. Sean $G$ un grafo con aristas valuadas y $T, T^{\prime}$ dos árboles generadores de $G$ con peso máximo. Entonces, existe una sucesión $T_{1} T_{2} \ldots T_{k}$ tal que $T_{1}=T, T_{k}=T^{\prime}, T_{i}$ es un árbol generador de peso máximo de $G$ para todo $1 \leq i \leq k$ y, para todo $2 \leq i \leq k, T_{i}$ puede ser obtenido a partir de $T_{i-1}$ agregándole una arista de $G$ y quitándole otra.

Teorema 4.8. Sea $G$ un grafo cordal. Entonces, existe un árbol compatible con $K(G)$ que no es un árbol clique de $G$ si y sólo si existen $S \in \mathcal{S}(G)$ y $C_{1}, C_{2} \in \mathcal{C}(G)$ tales que $C_{1} \cap C_{2} \subsetneq S$ y, para todo $C \in \mathcal{C}(G), C \cap S \neq \emptyset$ implica que $C \cap C_{1} \neq \emptyset$ y $C \cap C_{2} \neq \emptyset$.

Demostración. Supongamos que existe un árbol $T$ que es compatible con $K(G)$ pero que no es un árbol clique de $G$. Sea $T^{\prime}$ un árbol clique de $G$. Entonces, $T^{\prime}$ es compatible con $K(G)$ por la Proposición 4.1 y, como consecuencia del Teorema 4.3 y la Proposición 4.7, existe una sucesión de árboles compatibles con $K(G), T_{1} T_{2} \ldots T_{k}, T_{1}=T^{\prime}, T_{k}=T$, tal que todo árbol de la sucesión distinto de $T_{1}$ es construido a partir de su predecesor agregándole una arista y quitándole otra. Sea $i, 1 \leq i \leq k-1$, un número tal que $T_{i}$ es un árbol clique de $G$ y $T_{i+1}$ no lo es. Sean $C_{1} C_{2}$ la arista que es agregada y $C_{3} C_{4}$ la arista que es quitada para obtener a $T_{i+1}$ a partir de $T_{i}$. Entonces, $C_{3}, C_{4} \in T_{i}\left[C_{1}, C_{2}\right]$ (ver Figura 4.2), lo cual implica que $N_{K(G)}\left[C_{1}\right] \cap N_{K(G)}\left[C_{2}\right] \subseteq$ $N_{K(G)}\left[C_{3}\right] \cap N_{K(G)}\left[C_{4}\right]$. Además, se deduce del Teorema 4.3 que $\left|N_{K(G)}\left[C_{1}\right] \cap N_{K(G)}\left[C_{2}\right]\right|=$ $\left|N_{K(G)}\left[C_{3}\right] \cap N_{K(G)}\left[C_{4}\right]\right|$ y, por ende, las dos intersecciones son iguales (*). Como $T_{i}$ es un árbol clique, $C_{1} \cap C_{2} \subseteq C_{3} \cap C_{4}$. Pero, como $T_{i+1}$ no es un árbol clique, inferimos del Teorema 4.2 que $\left|C_{1} \cap C_{2}\right|<\left|C_{3} \cap C_{4}\right|$. Sea $S=C_{3} \cap C_{4}$. Entonces, por el Teorema 4.5, $S \in \mathcal{S}(G), C_{1} \cap C_{2} \subsetneq S$ y la condición de que, para todo $C \in \mathcal{C}(G), C \cap S \neq \emptyset$ implica que $C \cap C_{1} \neq \emptyset$ y $C \cap C_{2} \neq \emptyset$ se deduce de $(*)$.

Recíprocamente, supongamos que existen $S \in \mathcal{S}(G)$ y $C_{1}, C_{2} \in \mathcal{C}(G)$ tales que $C_{1} \cap C_{2} \subsetneq S$ y, para todo $C \in \mathcal{C}(G), C \cap S \neq \emptyset$ implica que $C \cap C_{1} \neq \emptyset$ y $C \cap C_{2} \neq \emptyset$. Sean $C_{3}, C_{4}$ un par separador de $G$ tal que $C_{3} \cap C_{4}=S$ y $T$ un árbol clique de $G$ tal que $C_{3} C_{4} \in E(T)$. Consideremos los siguientes casos:

(1) $C_{3}, C_{4} \in T\left[C_{1}, C_{2}\right]$ : la hipótesis y el hecho de que $T$ es compatible con $K(G)$ implican que $N_{K(G)}\left[C_{1}\right] \cap N_{K(G)}\left[C_{2}\right]=N_{K(G)}\left[C_{3}\right] \cap N_{K(G)}\left[C_{4}\right]$. Entonces, $T+C_{1} C_{2}-C_{3} C_{4}$ es compatible con $K(G)$ pero, como $\left|C_{1} \cap C_{2}\right|<\left|C_{3} \cap C_{4}\right|$, no es un árbol clique de $G$. 

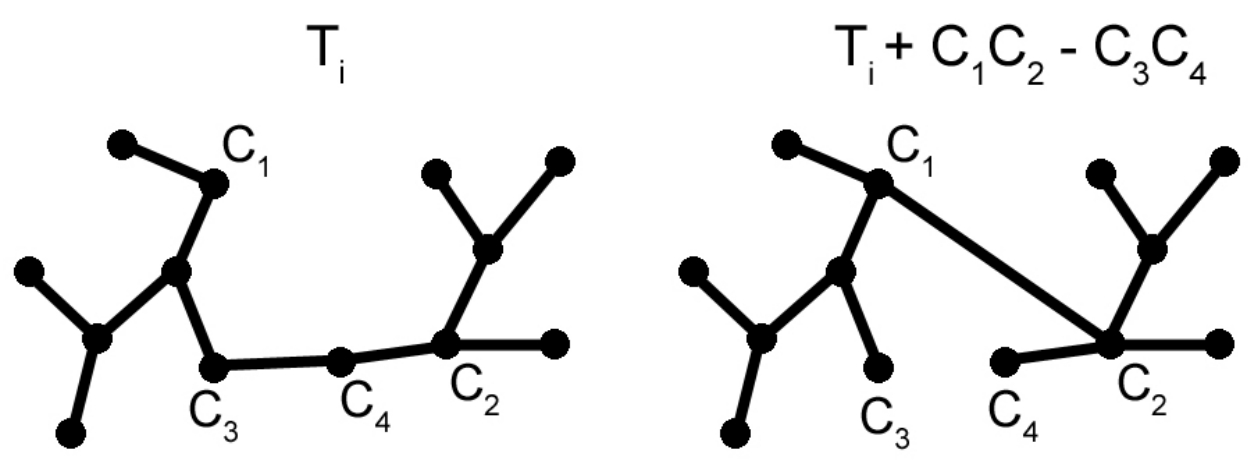

Figura 4.2: $\mathrm{Si} T_{i}+C_{1} C_{2}-C_{3} C_{4}$ es un árbol, entonces $C_{3}, C_{4} \in T_{i}\left[C_{1}, C_{2}\right]$.

(2) En caso contrario, $C_{1}$ y $C_{2}$ están en la misma componente conexa de $T-C_{3} C_{4}$. Esto implica que $C_{3} \in T\left[C_{1}, C_{4}\right] \cap T\left[C_{2}, C_{4}\right]$ o que $C_{4} \in T\left[C_{1}, C_{3}\right] \cap T\left[C_{2}, C_{3}\right]$ (ver Figura 4.3).
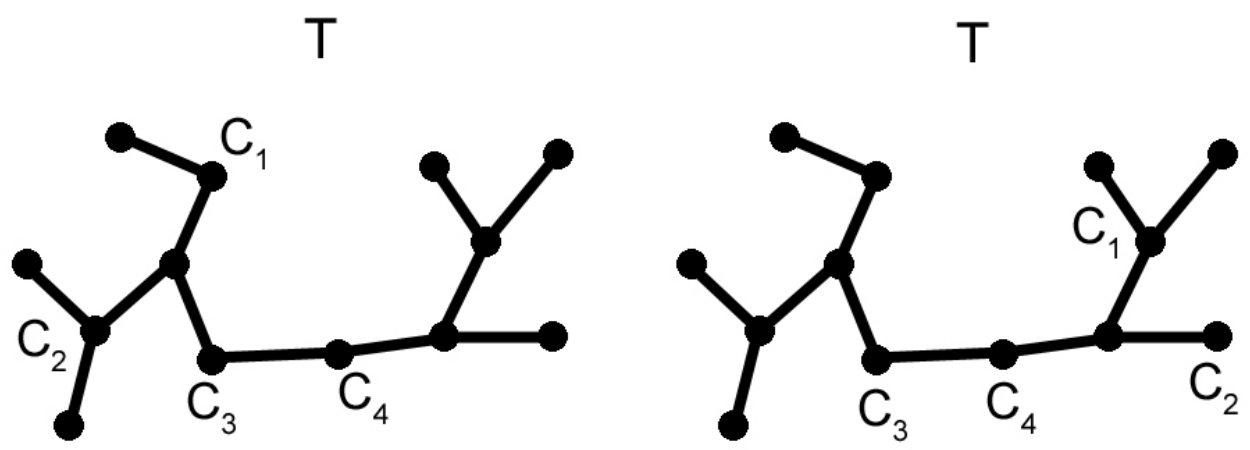

Figura 4.3: Si $C_{1}$ y $C_{2}$ están en la misma componente conexa de $T-C_{3} C_{4}$ que $C_{3}$, entonces $C_{3} \in T\left[C_{1}, C_{4}\right] \cap T\left[C_{2}, C_{4}\right]$ (izquierda). Si están en la misma componente conexa que $C_{4}$, entonces $C_{4} \in T\left[C_{1}, C_{3}\right] \cap T\left[C_{2}, C_{3}\right]$ (derecha).

Supongamos que $C_{3} \in T\left[C_{1}, C_{4}\right] \cap T\left[C_{2}, C_{4}\right]$. Entonces, $C_{1} \cap C_{4} \subseteq C_{3} \cap C_{4}$ y $C_{2} \cap C_{4} \subseteq C_{3} \cap C_{4}$. Si $C_{1} \cap C_{4}=C_{3} \cap C_{4}$ y $C_{2} \cap C_{4}=C_{3} \cap C_{4}, T^{\prime}:=T+C_{1} C_{4}-C_{3} C_{4}$ es un árbol clique de $G$. Como $C_{1} \in T^{\prime}\left[C_{2}, C_{4}\right], C_{2} \cap C_{4} \subseteq C_{1} \cap C_{2}$, lo cual implica que $C_{3} \cap C_{4} \subseteq C_{1} \cap C_{2}$, que es absurdo. Por consiguiente, $C_{1} \cap C_{4} \subsetneq C_{3} \cap C_{4}$ o $C_{2} \cap C_{4} \subsetneq C_{3} \cap C_{4}$. Además, para todo $C \in \mathcal{C}(G), C \cap S \neq \emptyset$ implica que $C \cap C_{1} \neq \emptyset, C \cap C_{2} \neq \emptyset, C \cap C_{3} \neq \emptyset$ y $C \cap C_{4} \neq \emptyset$. Si $C_{1} \cap C_{4} \subsetneq C_{3} \cap C_{4}$, entonces el hecho de que $C_{3}, C_{4} \in T\left[C_{1}, C_{4}\right]$ implica que podemos aplicar el caso (1) con $C_{1}, C_{4}$ en lugar de $C_{1}, C_{2}$. El caso en que $C_{2} \cap C_{4} \subsetneq C_{3} \cap C_{4}$ es análogo.

Si $C_{4} \in T\left[C_{1}, C_{3}\right] \cap T\left[C_{2}, C_{3}\right]$, la prueba es similar.

\subsection{Conjuntos que inducen subárboles y el concepto de base}

Visto que los árboles clique y los árboles compatibles son caracterizados por el hecho de que algunos conjuntos particulares inducen subárboles de ellos, es natural preguntarse qué otros conjuntos también inducen subárboles. Esta cuestión es lo que se estudiará a continuación y las 
conclusiones que se obtendrán resultarán ser muy útiles para profundizar nuestro conocimiento de los grafos básicamente cordales.

Sea $G$ un grafo. Si $G$ es cordal, entonces $\mathcal{S C}(G)$ denotará a la familia de todos los conjuntos $F$ tales que, para todo árbol clique $T$ de $G, T[F]$ es un subárbol de $T$. Por ejemplo, todo miembro de la familia dual de cliques de $G$ está en $\mathcal{S C}(G)$, lo cual es una consecuencia de la definición de árbol clique. Por la Proposición 3.2, $\mathcal{S C}(G)$ es una familia Helly.

Similarmente, si $G$ es dualmente cordal, $\mathcal{S D C}(G)$ denotará a la familia de todos los conjuntos $F$ tales que, para todo árbol $T$ compatible con $G, T[F]$ es un subárbol de $T$. Esta familia también es Helly y, entre sus miembros más conocidos, están los cliques, las vecindades cerradas y los separadores minimales de vértices de $G$, lo cual es consecuencia de las caracterizaciones de los grafos dualmente cordales que conocemos.

Estas definiciones y la Proposición 4.1 nos permiten deducir que, para $G$ cordal, $\mathcal{S D C}(K(G)) \subseteq$ $\mathcal{S C}(G)$.

No siempre es fácil hacer un listado de todos los miembros de $\mathcal{S C}(G)$ o $\mathcal{S D C}(G)$ porque no hay una cota polinomial para el cardinal de estas familias. A pesar de esto, se puede aspirar a obtener un procedimiento por el cual se generarían todos los miembros de estas familia con solamente conocer "algunos" de ellos.

Dada una familia $\mathcal{F}$ de conjuntos, se dice que la unión $\bigcup_{F \in \mathcal{F}} F$ es conexa si el grafo de intersección de $\mathcal{F}$ es conexo. Se denotará por $\bigcup_{F \in \mathcal{F}}^{c} F$. No es difícil ver que $\mathcal{S C}(G)$ y $\mathcal{S D C}(G)$ son cerradas con respecto a intersecciones y uniones conexas.

Supongamos que una familia $\mathcal{F}$ es cerrada con respecto a uniones conexas. Llamamos a una subfamilia $\mathcal{B}$ de $\mathcal{F}$ generadora si todo miembro de $\mathcal{F}$ con más de un elemento puede ser expresado como la unión conexa de algunos miembros de $\mathcal{B}$. Es $\mathcal{B}$ una base de $\mathcal{F}$ si, además, ninguna subfamilia propia de $\mathcal{B}$ genera a $\mathcal{F}$.

Una condición para determinar si un miembro de una familia está en alguna base es la siguiente:

Proposición 4.9. Sean $\mathcal{B}$ una base de $\mathcal{F}$ y $D \in \mathcal{F},|D|>1$. Son equivalentes:

(a) D está en $\mathcal{B}$.

(b) Para toda subfamilia $\mathcal{F}^{\prime}$ de $\mathcal{F}, D=\bigcup_{F \in \mathcal{F}^{\prime}}^{c} F$ implica que existe $F \in \mathcal{F}^{\prime}$ tal que $F=D$.

\section{Demostración.}

$(a) \Rightarrow(b)$ : Sea $\mathcal{F}^{\prime}$ una subfamilia de $\mathcal{F}$ tal que $D=\bigcup_{F \in \mathcal{F}^{\prime}}^{c} F$, y sean $F_{1}, \ldots, F_{n}$ los conjuntos no unitarios que están en $\mathcal{F}^{\prime}$, por lo que $D=\bigcup_{1 \leq i \leq n}^{c} F_{i}$ también. Para cada valor de $i$ entre 1 y $n$, sean $D_{i 1}, \ldots, D_{i m}$ miembros de $\mathcal{B}$ tales que $F_{i}=\bigcup_{1 \leq j \leq m}^{c} D_{i j}$. Por consiguiente, $D=\bigcup_{\substack{1 \leq i \leq n \\ 1 \leq j \leq m}}^{c} D_{i j}$. Si ningún $D_{i j}$ es igual a $D$, entonces la igualdad anterior implicaría que $\mathcal{B} \backslash\{D\}$ también genera a $\mathcal{F}$, contradiciendo que $\mathcal{B}$ es una base.

Sean entonces $i, j, 1 \leq i \leq n, 1 \leq j \leq m$, tales que $D_{i j}=D$. Como $D_{i j} \subseteq F_{i} \subseteq D, F_{i}=D$.

$(b) \Rightarrow(a)$ : Al ser $\mathcal{B}$ una base, sean $D_{1}, \ldots, D_{n}$ miembros de $\mathcal{B}$ tales que $D=\bigcup_{1 \leq i \leq n}^{c} D_{i}$. Por hipótesis, $D_{i}=D$ para algún valor de $i$. Luego, $D \in \mathcal{B}$. 
Como la condición (b) es independiente de la base que se esté considerando, lo siguiente se desprende de manera inmediata:

Corolario 4.10. $\mathcal{F}$ tiene una única base.

A continuación, se enuncian los principales resultados de esta sección. Primero se muestra la relación entre las dos familias que se acaban de definir y los grafos básicamente cordales. Luego, dado un grafo cordal $G$, se halla la base de $\mathcal{S C}(G)$.

Teorema 4.11. Sea $G$ un grafo cordal. Son equivalentes:

(a) Todo árbol compatible con $K(G)$ es un árbol clique de $G$.

(b) $\mathcal{S C}(G)=\mathcal{S D C}(K(G))$.

(c) $\mathcal{S C}(G)$ y $\mathcal{S D C}(K(G))$ tienen la misma base.

Demostración.

$(a) \Rightarrow(b)$ : La Proposición 4.1 y la hipótesis implican que, para todo árbol $T, T$ es un árbol clique de $G$ si y sólo si $T$ es compatible con $K(G)$. Por lo tanto, las definiciones de $\mathcal{S C}(G)$ y $\mathcal{S D C}(K(G))$ implican que estas familias son iguales.

$(b) \Rightarrow(a)$ : La igualdad entre $\mathcal{S C}(G)$ y $\mathcal{S D C}(K(G))$ implica que $D \mathcal{C}(G) \subseteq \mathcal{S D C}(K(G))$. Por lo tanto, todo árbol compatible con $K(G)$ es un árbol clique de $G$.

$(b) \Leftrightarrow(c)$ : Trivial.

El nombre básicamente cordal para la clase de grafos que estamos estudiando se inspira en la parte $(c)$ del Teorema 4.11.

Proposición 4.12. Sean $G$ un grafo cordal y $A \in \mathcal{S C}(G)$. Si $C_{1}, C_{2}$ es un par separador contenido en $A$, entonces $\mathcal{C}_{C_{1} \cap C_{2}} \subseteq A$.

Demostración. Sea $C$ un elemento cualquiera de $\mathcal{C}_{C_{1} \cap C_{2}}$. Si $C=C_{1}$ o $C=C_{2}$, entonces es claro que $C \in A$. Supongamos ahora que $C \neq C_{1}$ y $C \neq C_{2}$. Sea $T$ un árbol clique de $G$ tal que $C_{1} C_{2} \in E(T)$. Podemos suponer también sin pérdida de generalidad que $C_{2} \in T\left[C, C_{1}\right]$, por lo que $C \cap C_{1} \subseteq C_{1} \cap C_{2}$. Como $C \in \mathcal{C}_{C_{1} \cap C_{2}}, C_{1} \cap C_{2} \subseteq C \cap C_{1}$, e inferimos del Teorema 4.2 que $T^{\prime}:=T-C_{1} C_{2}+C C_{1}$ es un árbol clique de $G$. Ya que $A \in \mathcal{S C}(G), T^{\prime}[A]$ es un subárbol de $T^{\prime}$. Además, $C \in T^{\prime}\left[C_{1}, C_{2}\right]$ y $C_{1}, C_{2} \in A$. Luego, $C \in A$.

De esto se sigue la inclusión $\mathcal{C}_{C_{1} \cap C_{2}} \subseteq A$.

Teorema 4.13. Sea $G$ un grafo cordal. Entonces, $\left\{\mathcal{C}_{S}, S \in \mathcal{S}(G)\right\}$ es la base de $\mathcal{S C}(G)$.

Demostración. Sean $A$ un miembro cualquiera de $\mathcal{S C}(G)$ tal que $|A|>1$ y $T$ un árbol clique de $G$. Denotemos por $e_{1}, e_{2}, \ldots, e_{k}$ a todas las aristas de $T[A]$ y por $S_{i}, i=1, \ldots, k$, al separador minimal de vértices de $G$ igual a la intersección de los extremos de $e_{i}$. Por la Proposición 4.12, $\mathcal{C}_{S_{i}} \subseteq A, i=1, \ldots, k$. Esto implica que $\bigcup_{i=1}^{k} \mathcal{C}_{S_{i}} \subseteq A$. Como también es cierto que $\mathcal{C}_{S_{i}}$ contiene a los extremos de $e_{i}, i=1, \ldots, k$, y que $T[A]$ es un subárbol, deducimos que $A \subseteq \bigcup_{i=1}^{k} \mathcal{C}_{S_{i}}$. Luego, $A=\bigcup_{1 \leq i \leq k}^{c} \mathcal{C}_{S_{i}}$

Por consiguiente, $\left\{\mathcal{C}_{S}, S \in \mathcal{S}(G)\right\}$ genera a $\mathcal{S C}(G)$. 
Sean $S$ un separador minimal de vértices fijo y $C_{1} C_{2}$ una arista de $T$ tal que $C_{1} \cap C_{2}=S$. Supongamos que $\left\{M_{1}, . ., M_{n}\right\}$ es una subfamilia de $\mathcal{S C}(G)$ tal que $\mathcal{C}_{S}=\bigcup_{1 \leq i \leq n}^{c} M_{i}$ y sean $T_{1}$ y $T_{2}$ los subárboles que se obtienen al remover la arista $C_{1} C_{2}$ de $T\left[\mathcal{C}_{S}\right]$, con $C_{1} \in V\left(T_{1}\right)$ y $C_{2} \in V\left(T_{2}\right)$. Como $\bigcup_{i=1}^{n} M_{i}$ es conexa, debe existir un índice $j, 1 \leq j \leq n$, tal que $M_{j} \cap V\left(T_{1}\right) \neq \emptyset$ y $M_{j} \cap V\left(T_{2}\right) \neq \emptyset$. Al ser $T\left[M_{j}\right]$ un subárbol de $T\left[\mathcal{C}_{S}\right], C_{1}, C_{2} \in M_{j}$. Apliquemos ahora la Proposición 4.12 para obtener que $\mathcal{C}_{C_{1} \cap C_{2}} \subseteq M_{j}$, es decir, $\mathcal{C}_{S} \subseteq M_{j}$ y que, por ende, $\mathcal{C}_{S}=M_{j}$. Luego, por la Proposición $4.9, \mathcal{C}_{S}$ es un miembro de la base de $\mathcal{S C}(G)$.

Por lo tanto, $\left\{\mathcal{C}_{S}, S \in \mathcal{S}(G)\right\}$ es la base de $\mathcal{S C}(G)$.

Si notamos que el número de miembros de $\mathcal{C}(G)$ y $\mathcal{S}(G)$ es del orden $O(|V(G)|)$ porque $G$ es cordal, concluimos que calcular la base de $\mathcal{S C}(G)$ es algo que puede realizarse de modo eficiente en tiempo polinomial.

Si llamamos dimensión de $G$ al número de miembros de la base de $\mathcal{S C}(G)$, el Teorema 4.13 implica que dicha dimensión es igual a $|\mathcal{S}(G)|$, cantidad la cual, por los Teoremas 4.4, 4.5 y 4.6, es menor o igual que el número de cliques menos uno. Por otro lado, si $G$ es dualmente cordal, llamemos dimensión dual de $G$ al número de miembros de la base de $\mathcal{S D C}(G)$.

Se desprende claramente del Teorema 4.11 que, si $G$ es básicamente cordal, la dimensión de $G$ es igual a la dimensión dual de $K(G)$. Pero la recíproca no es necesariamente verdadera.
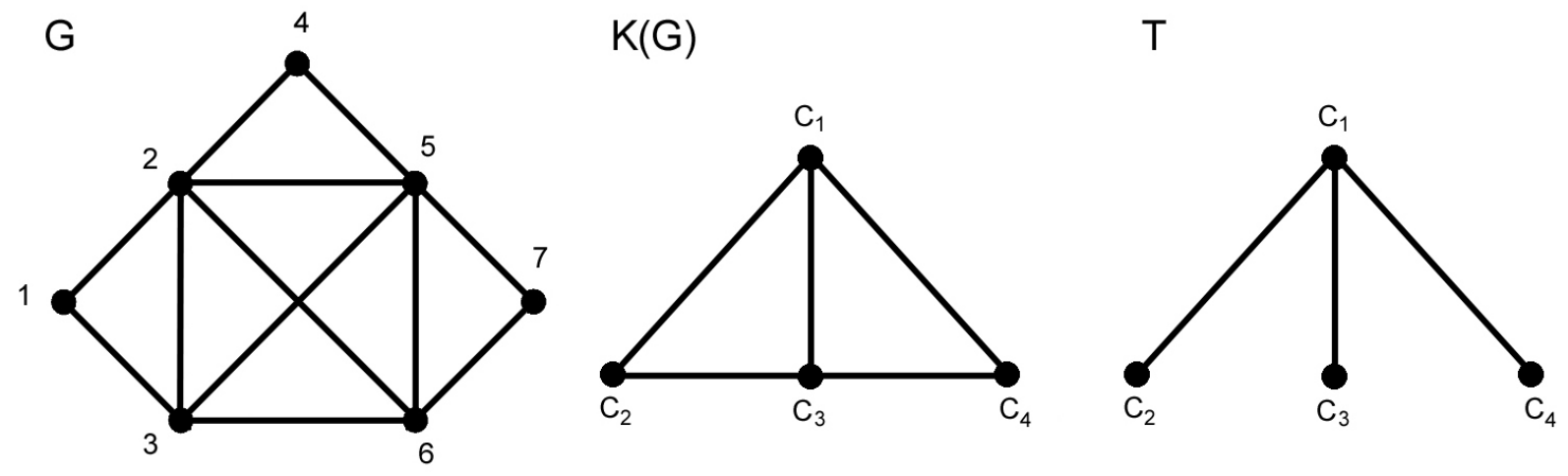

Figura 4.4: Un grafo cordal $G$, su grafo clique y un árbol $T$ compatible con $K(G)$ que no es un árbol clique de $G$.

Consideremos al grafo cordal $G$ de la Figura 4.4. Sus cliques son $C_{1}=\{2,4,5\}, C_{2}=\{1,2,3\}$, $C_{3}=\{2,3,5,6\}$ y $C_{4}=\{5,6,7\}$. Los pares separadores son $C_{1}, C_{3} ; C_{2}, C_{3}$ y $C_{3}, C_{4}$, por lo que $\mathcal{S}(G)=\{\{2,3\},\{2,5\},\{5,6\}\}$. Por el Teorema 4.13, la base de $\mathcal{S C}(G)$ es $\left\{\left\{C_{1}, C_{3}\right\},\left\{C_{2}, C_{3}\right\}\right.$, $\left.\left\{C_{3}, C_{4}\right\}\right\}$. Debido a la simplicidad de $K(G)$, no es difícil verificar que la base de $\mathcal{S D C}(K(G))$ es $\left\{\left\{C_{1}, C_{2}, C_{3}\right\},\left\{C_{1}, C_{3}\right\},\left\{C_{1}, C_{3}, C_{4}\right\}\right\}$. En este caso, tanto la dimensión de $G$ como la dimensión dual de $K(G)$ son iguales a 3 , pero las bases son diferentes, por lo que existe un árbol compatible con $K(G)$ que no es un árbol clique de $G$. Un ejemplo de árbol así es $T$, también mostrado en la Figura 4.4. No es un árbol clique porque ni $\mathcal{C}_{3}$ ni $\mathcal{C}_{6}$ inducen subárboles de $T$. 


\subsection{Más resultados acerca de los grafos básicamente cordales y dualmente cordales}

En esta sección, continuaremos con el trabajo necesario para hallar una nueva caracterización de los grafos básicamente cordales. En primer lugar, dado un grafo dualmente cordal $G$, se caracterizarán en el Teorema 4.15 todos los grafos básicamente cordales con grafo clique igual a $G$. Esto nos permitirá demostrar varias propiedades de los grafos dualmente cordales. En particular, se obtendrá una nueva caracterización de los grafos dualmente cordales basada en los árboles compatibles (Teorema 4.22) y se hallará la base de $\mathcal{S D C}(G)$ (Teorema 4.18).

Finalmente, el saber cómo hallar la base tanto al trabajar con los grafos cordales como con los dualmente cordales nos dará una caracterización de los grafos básicamente cordales, la cual será luego aplicada para probar un par de propiedades adicionales de estos grafos.

Diremos que un grafo cordal $H$ está en correspondencia con $G$ si $H$ es básicamente cordal y $K(H)=G$. Los pasos a continuación serán para hallar todos los grafos cordales en correspondencia con $G$.

Proposición 4.14. [14] Sea $\mathcal{F}$ una familia Helly separadora. Entonces, $\mathcal{C}(L(\mathcal{F}))=D \mathcal{F}$.

Teorema 4.15. Sean $G$ un grafo dualmente cordal y $H$ un grafo cordal. Entonces, $H$ está en correspondencia con $G$ si y sólo si $H$ es el grafo de intersección de una subfamilia separadora de $\mathcal{S D C}(G)$ cuya dos sección es igual a $G$.

Demostración. Supongamos que $H$ está en correspondencia con $G$. Sabemos que $H$ es el grafo de intersección de $\left\{\mathcal{C}_{v}\right\}_{v \in V(H)}$, que es una subfamilia de $\mathcal{S C}(H)$. No es difícil verificar que la dos sección de esta familia es $K(H)$, que es igual a $G$, y que es separadora. Además, por el Teorema $4.11, \mathcal{S C}(H)=\mathcal{S D C}(K(H))=\mathcal{S D C}(G)$, por lo que $\left\{\mathcal{C}_{v}\right\}_{v \in V(H)} \subseteq \mathcal{S D C}(G)$.

Recíprocamente, supongamos que $H$ es el grafo de intersección de una familia separadora $\mathcal{F}$ tal que $S(\mathcal{F})=G$ y con todos sus miembros en $\mathcal{S D C}(G)$. Por el Teorema 1.4, $H$ es cordal.

Veamos ahora que $K(H)$ es isomorfo a $G$. Al ser $\mathcal{F}$ Helly y separadora, podemos aplicar la Proposición 4.14 para obtener que $\mathcal{C}(H)=\mathcal{C}(L(\mathcal{F}))=D \mathcal{F}$. Además, para todos $u, v \in V(G)$,

$$
\begin{gathered}
D_{u} D_{v} \in E(K(H)) \Leftrightarrow D_{u} \cap D_{v} \neq \emptyset \Leftrightarrow \exists F \in \mathcal{F}, F \in D_{u} \wedge F \in D_{v} \Leftrightarrow \\
\exists F \in \mathcal{F}, u \in F \wedge v \in F \Leftrightarrow u v \in E(G)
\end{gathered}
$$

justificándose la última equivalencia en el hecho de que la dos sección de $\mathcal{F}$ es $G$. Luego, la función $f: V(G) \rightarrow V(K(H))$ dada por $f(v)=D_{v}$ es un isomorfismo entre $G$ y $K(H)$.

Para cada $F \in \mathcal{F}$, consideremos el miembro $\mathcal{C}_{F}$ de $D \mathcal{C}(H)$. Se tiene que $\mathcal{C}_{F}=\{C \in$ $\mathcal{C}(H): F \in C\}=\left\{D_{v} \in D \mathcal{F}: v \in F\right\}$. Como $F \in \mathcal{S D C}(G),\left\{D_{v} \in D \mathcal{F}: v \in F\right\} \in$ $\mathcal{S D C}(K(H))$. Por consiguiente, $D \mathcal{C}(H) \subseteq \mathcal{S D C}(K(H))$, lo cual hace que todo árbol compatible con $K(H)$ sea un árbol clique de $H$. Esto completa la demostración.

Consideremos como ejemplo de este teorema al grafo $K(G)$ de la Figura 4.4. Sabemos que $G$ no está en correspondencia con $K(G)$. Busquemos un grafo en correspondencia con $K(G)$. $\left\{C_{1}, C_{2}, C_{3}\right\}$ y $\left\{C_{1}, C_{3}, C_{4}\right\}$ forman una subfamilia de $\mathcal{S D C}(K(G))$ cuya dos sección es $K(G)$, pero no es separadora. Sea entonces $\mathcal{F}=\left\{\left\{C_{1}\right\},\left\{C_{2}\right\},\left\{C_{3}\right\},\left\{C_{4}\right\},\left\{C_{1}, C_{2}, C_{3}\right\},\left\{C_{1}, C_{3}, C_{4}\right\}\right\}$. Por el Teorema 4.15, el grafo de intersección de esta familia (ver Figura 4.5) está en correspondencia con $K(G)$. 

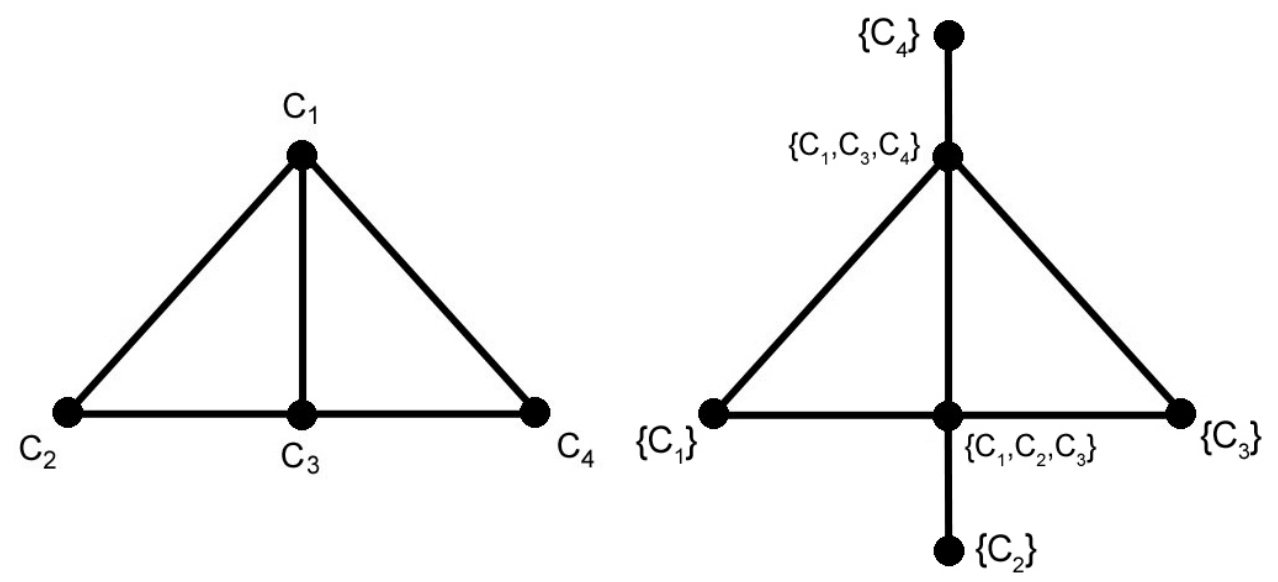

Figura 4.5: El grafo dualmente cordal $K(G)$ de la Figura 4.4 y, a la derecha, un grafo cordal cuyos árboles clique son exactamente los árboles compatibles de $K(G)$.

El Teorema 4.15 nos provee del marco ideal para que muchas de las propiedades vistas antes en este capítulo puedan ser utilizadas para obtener propiedades de los árboles compatibles similares a las de los árboles clique, y también obtener algunas cosas más.

Teorema 4.16. Sean $G$ un grafo dualmente cordal, $\mathcal{F}$ una subfamilia separadora de $\mathcal{S D C}(G)$ tal que $S(\mathcal{F})=G$ y $D \mathcal{F}=\left\{D_{v}\right\}_{v \in V(G)}$. Entonces:

(a) Dados $u, v \in V(G)$, existe un árbol $T$ compatible con $G$ y tal que uv $\in E(T)$ si y sólo si $D_{u}$ y $D_{v}$ forman un par separador de $L(\mathcal{F})$.

(b) Si $T$ es un árbol compatible con $G$, existe un árbol $T^{\prime}$ compatible con $G$ y tal que uv $\in E\left(T^{\prime}\right)$ si y sólo si existen dos vértices $x$ e y adyacentes en $T\langle u, v\rangle$ tales que $D_{x} \cap D_{y}=D_{u} \cap D_{v}$.

(c) Asignemos a cada uv $\in E(G)$ el número $\left|D_{u} \cap D_{v}\right|$ para así obtener el grafo valuado $G^{w}$. Entonces, un árbol $T$ es compatible con $G$ si y sólo si es un árbol generador de $G^{w}$ de peso máximo, siendo el peso igual a $\sum_{F \in \mathcal{F}}|F|-|\mathcal{F}|$.

(d) Si $T$ es compatible con $G, A \in \mathcal{S D C}(G)$, uv $\in E(T) y\{u, v\} \subseteq A$, entonces $\bigcap_{F \in D u \cap D v} F \subseteq A$.

(e) Si $T$ es compatible con $G,\left\{\bigcap_{F \in D_{u} \cap D_{v}} F: u v \in E(T)\right\}$ es la base de $\mathcal{S D C}(G)$.

Demostración. Por la Proposición 4.14, $\mathcal{C}(L(\mathcal{F}))=D \mathcal{F}$. Entonces:

(a) Usar el Teorema 4.15 y luego aplicar el Teorema 4.5 a $L(\mathcal{F})$.

(b) Usar el Teorema 4.15 y luego aplicar el Teorema 4.6 a $L(\mathcal{F})$.

(c) Usar el Teorema 4.15 y luego aplicar el Teorema 4.2 a $L(\mathcal{F})$, notando que

$$
\begin{gathered}
\sum_{C \in \mathcal{C}(L(\mathcal{F}))}|C|-|V(L(\mathcal{F}))|=\sum_{v \in V(G)}\left|D_{v}\right|-|\mathcal{F}|= \\
\sum_{v \in V(G)} \sum_{F \in \mathcal{F}}|\{v\} \cap F|-|\mathcal{F}|=\sum_{F \in \mathcal{F}} \sum_{v \in V(G)}|\{v\} \cap F|-|\mathcal{F}|=\sum_{F \in \mathcal{F}}|F|-|\mathcal{F}|
\end{gathered}
$$


(d) Aplicar la parte (a), y los Teoremas 4.15 y 4.11 y la Proposición 4.12 a $L(\mathcal{F})$, notando que

$$
\begin{aligned}
& \mathcal{C}_{D_{u} \cap D_{v}}=\left\{C \in \mathcal{C}(L(\mathcal{F})): D_{u} \cap D_{v} \subseteq C\right\}=\left\{D_{w}: D_{u} \cap D_{v} \subseteq D_{w}\right\}= \\
& \left\{D_{w}: \forall F \in \mathcal{F}, F \in D_{u} \cap D_{v} \rightarrow w \in F\right\}=\left\{D_{w}: w \in \bigcap_{F \in D_{u} \cap D_{v}} F\right\}
\end{aligned}
$$

(e) La parte (d) implica que $\left\{\bigcap_{F \in D_{u} \cap D_{v}} F: u v \in E(T)\right\}$ genera a $\mathcal{S D C}(G)$. La minimalidad es una consecuencia de la parte (a) y los Teoremas $4.15,4.11$ y 4.13 aplicados a $L(\mathcal{F})$.

Un ejemplo típico de una familia con las características mencionadas en el Teorema 4.16 es la que consiste de todos los cliques de $G$ y todos los conjuntos unitarios de vértices. Si a ella se le aplican algunos de los resultados del Teorema 4.16, se obtienen las siguientes conclusiones:

Teorema 4.17. Sea $G$ un grafo dualmente cordal. Asignemos a cada arista uv $\in E(G)$ el número $\left|\mathcal{C}_{u} \cap \mathcal{C}_{v}\right|$ para obtener asi el grafo valuado $G^{w}$. Entonces, un árbol $T$ es compatible con $G$ si y sólo si es un árbol generador de peso máximo de $G^{w}$ con peso total igual a $\sum_{C \in \mathcal{C}(G)}|C|-|\mathcal{C}(G)|$.

Demostración. Sea $\mathcal{F}=\mathcal{C}(G) \cup\{\{v\}: v \in V(G)\}$. Applicar la parte (c) del Teorema 4.16 a esta familia y notar que, para toda $u v \in E(G),\left|D_{u} \cap D_{v}\right|=\left|\mathcal{C}_{u} \cap \mathcal{C}_{v}\right|$ y

$$
\sum_{F \in \mathcal{F}}|F|-|\mathcal{F}|=\sum_{C \in \mathcal{C}(G)}|C|+|V(G)|-(|\mathcal{C}(G)|+|V(G)|)=\sum_{C \in \mathcal{C}(G)}|C|-|\mathcal{C}(G)|
$$

El Teorema 4.17 es sabido desde hace varios años [13] y fue probado de manera independiente por varios autores.

Teorema 4.18. Sean $G$ un grafo dualmente cordal y $T$ un árbol compatible con $G$. Entonces, $\left\{\bigcap_{C \in \mathcal{C}_{u} \cap \mathcal{C}_{v}} C: u v \in E(T)\right\}$ es la base de $\operatorname{SDC}(G)$.

Demostración. Aplicar la parte (e) del Teorema 4.16 a $\mathcal{F}$ definida como en el teorema anterior. Notar nuevamente que $D_{u} \cap D_{v}=\mathcal{C}_{u} \cap \mathcal{C}_{v}$.

Como ejemplo, consideremos de nuevo el grafo $G$ de la Figura 4.4. $G$ es también dualmente cordal y uno de sus árboles compatibles aparece en la Figura 4.6. Usemos ahora las aristas de ese árbol para obtener la base de $\mathcal{S D C}(G)$. Consideremos a la arista 12 de $T$. El único clique que contiene a 1 y a 2 es $\{1,2,3\}$, por lo que este conjunto está en la base. Consideremos ahora a la arista 23 . Los cliques que contienen tanto a 2 como a 3 son $\{1,2,3\}$ y $\{2,3,5,6\}$. La intersección de ellos es $\{2,3\}$. Por lo tanto, este conjunto también está en la base. Similarmente, si consideramos las aristas restantes del árbol compatible, resulta que la base se completa con los conjuntos $\{2,5\},\{5,6\},\{2,4,5\}$ y $\{5,6,7\}$.

Hallar la base en el ejemplo fue fácil porque el grafo era pequeño, pero puede dificultarse calcular la base de grafos dualmente cordales cada vez más grandes, ya que no existe ninguna cota polinomial para el número de cliques de un grafo dualmente cordal. Por ejemplo, dado un 

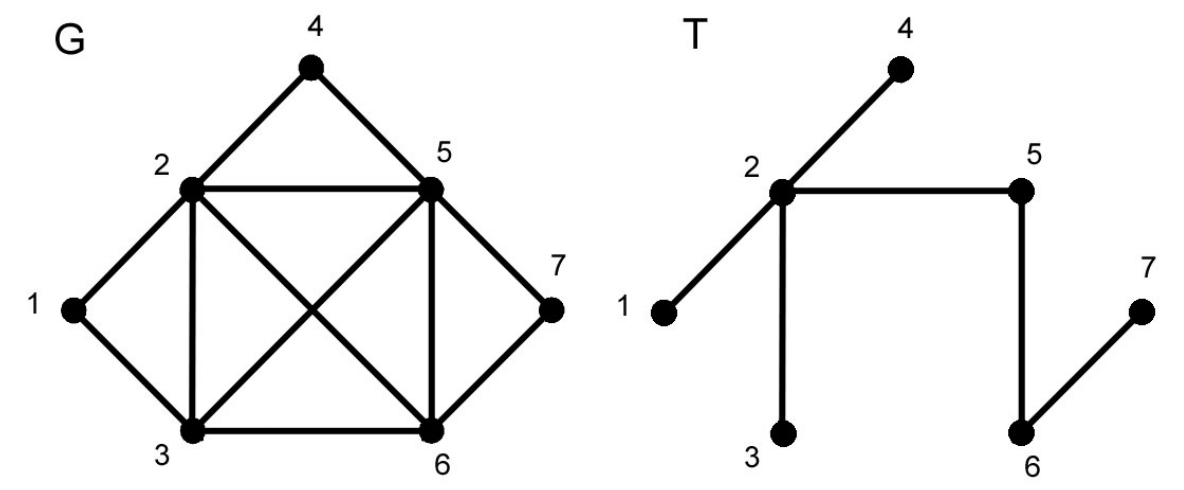

Figura 4.6: Un grafo dualmente cordal y uno de sus árboles compatibles.

entero positivo $n$, definamos a $n K_{2}$ como el grafo que posee $n$ componentes conexas, cada una de ellas con dos vértices. El complemento de $n K_{2}$ es un grafo con $2^{n}$ cliques, y el grafo obtenido a partir de $\overline{n K_{2}}$ agregándole un vértice universal es dualmente cordal y con igual cantidad de cliques.

Sin embargo, una conexión con las vecindades cerradas revela que es más simple de lo que se podría esperar:

Proposición 4.19. Sean $u$ y $v$ vértices adyacentes de un grafo $G$. Entonces, $\bigcap_{C \in \mathcal{C}_{u} \cap \mathcal{C}_{v}} C=$ $\bigcap_{w \in N[u] \cap N[v]} N[w]$.

Demostración. Probemos la doble inclusión.

Sean $x \in \bigcap_{C \in \mathcal{C}_{u} \cap \mathcal{C}_{v}} C \mathrm{y} w \in N[u] \cap N[v]$. Entonces, $\{u, v, w\}$ es un conjunto completo y existe un clique $C_{1}$ tal que $\{u, v, w\} \subseteq C_{1}$, lo cual implica que $C_{1} \in \mathcal{C}_{u} \cap \mathcal{C}_{v}$ y que, por ende, $x \in C_{1}$. Luego, $x \in N[w]$. Podemos concluir que $\bigcap_{C \in \mathcal{C}_{u} \cap \mathcal{C}_{v}} C \subseteq \bigcap_{w \in N[u] \cap N[v]} N[w]$.

Recíprocamente, sean $x \in \bigcap_{w \in N[u] \cap N[v]} N[w]$ y $C \in \mathcal{C}_{u} \cap \mathcal{C}_{v}$. Entonces, $C \subseteq N[u] \cap N[v]$ y, por definición de $x, x$ está en la vecindad cerrada de todo elemento de $C$. En consecuencia, $x \in C$. Podemos inferir de ese razonamiento que $\bigcap_{w \in N[u] \cap N[v]} N[w] \subseteq \bigcap_{C \in \mathcal{C}_{u} \cap \mathcal{C}_{v}} C$.

Otra alternativa para calcular la base de $\mathcal{S D C}(G)$ es reemplazar a $\mathcal{C}(G)$ por una subfamilia $\mathcal{F}$ cuyos miembros son seleccionados de la siguiente manera: para cada arista $u v$ de $G$, elegir $C \in \mathcal{C}(G)$ tal que $\{u, v\} \subseteq C$. Claramente $|\mathcal{F}| \leq|E(G)|$ y $S(\mathcal{F})=\mathcal{C}(G)$.

Usemos los últimos dos resultados para obtener una caracterización de los grafos dualmente cordales que generalice aquella que dice que un grafo es dualmente cordal si y sólo si existe un árbol generador $T$ tal que todo clique del grafo induce un subárbol de $T$.

Decimos que un conjunto $A$ de vértices de un grafo $G$ es booleano positivo si puede ser obtenido a partir de repetidas intersecciones y uniones de vecindades cerradas. Es booleano positivo conexo si la unión conexa reemplaza a la unión común. 
Una combinación del Teorema 4.18 y la Proposición 4.19 revela que los miembros de la base de $\mathcal{S D C}(G)$, para $G$ dualmente cordal, son booleanos positivos conexos, y lo mismo puede decirse sobre las uniones conexas de ellos. Como la vecindad cerrada de todo vértice induce un subárbol de todo árbol compatible del grafo, concluimos que:

Teorema 4.20. Sean $G$ un grafo dualmente cordal y $A \subseteq V(G)$. Entonces, $A \in \mathcal{S D C}(G)$ si y sólo si es booleano positivo conexo.

Veamos ahora cuál es la caracterización.

Teorema 4.21. Sean $G$ un grafo, $T$ un árbol generador de $G$ y $\mathcal{F}$ una familia de subconjuntos booleanos positivos conexos de $V(G)$ tal que $S(\mathcal{F})=G$. Entonces, son equivalentes:

(a) $T$ es compatible con $G$.

(b) Todo miembro de $\mathcal{F}$ induce un subárbol de $T$.

Demostración.

$(a) \Rightarrow(b)$ : Como $T$ es compatible con $G$, este grafo es dualmente cordal. Por el Teorema 4.20, todo miembro de $\mathcal{F}$ está en $\mathcal{S D C}(G)$, por lo que induce un subárbol de $T$.

$(b) \Rightarrow(a)$ : Sean $u$ y $v$ dos vértices adyacentes en $G$ y $w \in T(u, v)$. Como $S(\mathcal{F})=G$, podemos tomar $F \in \mathcal{F}$ tal que $\{u, v\} \subseteq F$. Además, debido a que $T[F]$ es un subárbol de $T, w \in F$. Por lo tanto, $w$ es adyacente a $u$ y a $v$ en $S(\mathcal{F})$, es decir, $u w \in E(G)$ y $v w \in E(G)$. Luego, por el Teorema 1.15, $T$ es compatible con $G$.

Corolario 4.22. Sean $G$ un grafo y $\mathcal{F}$ una familia de subconjuntos booleanos positivos conexos de $V(G)$ tal que $S(\mathcal{F})=G$. Entonces, $G$ es dualmente cordal si y sólo si existe un árbol generador $T$ de $G$ tal que todo miembro de $\mathcal{F}$ induce un subárbol de $T$.

Retomemos el estudio de los grafos básicamente cordales. Ahora que sabemos cómo calcular la base de $\mathcal{S D C}(G)$, podemos obtener también una nueva caracterización de los grafos básicamente cordales. Antes de enunciarla, se verán algunos lemas.

Lema 4.23. Sean $G$ un grafo cordal y $C_{1}, C_{2}$ un par separador de $G$. Si $C$ es un clique tal que $C \cap C_{1} \neq \emptyset$ y $C \cap C_{2} \neq \emptyset$, entonces $C \cap C_{1} \cap C_{2} \neq \emptyset$.

Demostración. Sea $T$ un árbol clique de $G$ tal que $C_{1} C_{2} \in E(T)$. Entonces, $C_{1} \in T\left[C, C_{2}\right]$ o $C_{2} \in T\left[C, C_{1}\right]$. En el primer caso, $C \cap C_{2} \subseteq C_{1} \cap C_{2}$. En el segundo, $C \cap C_{1} \subseteq C_{1} \cap C_{2}$. En ambos casos, $C \cap C_{1} \cap C_{2} \neq \emptyset$.

Supongamos ahora que $G$ es un grafo dualmente cordal y que tenemos un árbol compatible $T$ con sus aristas ordenadas. Si consideramos la parte (e) del Teorema 4.16, podría ocurrir que, dependiendo de la familia $\mathcal{F}$ que se utilice, los miembros de la base de $\mathcal{S D C}(G)$ se obtengan en diferentes órdenes posibles. No es el caso, como el siguiente lema demuestra.

Lema 4.24. Sean $G$ un grafo dualmente cordal, $T$ un árbol compatible con $G, u v \in E(T)$ y $\mathcal{F}$ cualquier subfamilia separadora de $\mathcal{S D C}(G)$ tal que $S(\mathcal{F})=G$. Entonces, $B_{\mathcal{F}}:=\bigcap_{F \in D_{u} \cap D_{v}} F$ no depende de la elección de $\mathcal{F}$. 
Demostración. Sean $\mathcal{F}$ y $\mathcal{F}^{\prime}$ subfamilias separadoras de $\mathcal{S D C}(G)$ tales que la dos sección de cada una es igual a $G$. El conjunto $\{u, v\}$ está contenido tanto en $B_{\mathcal{F}}$ como en $B_{\mathcal{F}^{\prime}}$, por lo que la parte (d) del Teorema 4.16 puede ser aplicada a $\mathcal{F}$ y a $\mathcal{F}^{\prime}$ para concluir que $B_{\mathcal{F}} \subseteq B_{\mathcal{F}^{\prime}}$ y $B_{\mathcal{F}^{\prime}} \subseteq B_{\mathcal{F}}$, respectivamente. Luego, $B_{\mathcal{F}}=B_{\mathcal{F}^{\prime}}$.

Dado un grafo $G$ y $S \in \mathcal{S}(G)$, definamos a $B_{S}$ como el conjunto de cliques de $G$ que intersecan a todo clique que interseca a $S$, es decir, $B_{S}=\bigcap_{C \cap S \neq \emptyset} N_{K(G)}[C]$. Este conjunto no debe ser confundido con el $B_{\mathcal{F}}$ del lema anterior. Se prueba ahora lo siguiente:

Lema 4.25. Sea $G$ un grafo cordal. Entonces, $\left\{B_{S}: S \in \mathcal{S}(G)\right\}$ es la base de $\mathcal{S D C}(K(G))$.

Demostración. Sean $S$ un separador minimal de vértices de $G$ y $C_{1}, C_{2}$ un par separador tal que $S=C_{1} \cap C_{2}$. Entonces, por el Lema 4.23, $\bigcap_{C \cap S \neq \emptyset} N_{K(G)}[C]=\bigcap_{C \in N_{K(G)}\left[C_{1}\right] \cap N_{K(G)}\left[C_{2}\right]} N_{K(G)}[C]$. Ahora apliquemos la Proposición 4.19 para concluir que $B_{S}=\bigcap_{\substack{D \in \mathcal{C}(K(G)) \\ C_{1}, C_{2} \in D}} D$.

Finalmente, tomemos un árbol clique $T$ de $G$ y completemos la demostración aplicando la Proposición 4.1 y los Teoremas 4.4, 4.5, 4.6 y 4.18.

Si $G$ es un grafo básicamente cordal, deducimos de los Teoremas 4.11 y 4.13 y el Lema 4.25 que $\left\{B_{S}: S \in \mathcal{S}(G)\right\}=\left\{\mathcal{C}_{S}: S \in \mathcal{S}(G)\right\}$. Entonces, para cada $S \in \mathcal{S}(G)$, existe $S^{\prime} \in \mathcal{S}(G)$ tal que $B_{S}=\mathcal{C}_{S^{\prime}}$. En principio, $S^{\prime}$ no necesita ser igual a $S$. Sin embargo, la igualdad siempre se cumple, conduciendo a la siguiente caracterización:

Teorema 4.26. Sea $G$ un grafo cordal. Entonces, $G$ es básicamente cordal si y sólo si, para todo $S \in \mathcal{S}(G), B_{S}=\mathcal{C}_{S}$.

Demostración. Supongamos que, para todo $S \in \mathcal{S}(G), B_{S}=\mathcal{C}_{S}$. Entonces, por el Teorema 4.13 y el Lema 4.25 , las bases de $\mathcal{S C}(G)$ y $\mathcal{S D C}(K(G))$ son iguales. Se sigue del Teorema 4.11 que $G$ es básicamente cordal.

Recíprocamente, supongamos que $G$ es un grafo básicamente cordal. Sean $S \in \mathcal{S}(G), T$ un árbol clique de $G$ y $C_{1} C_{2} \in E(T)$ tal que $C_{1} \cap C_{2}=S$. Consideremos a las familias $\mathcal{F}_{1}=$ $\mathcal{C}(K(G)) \cup\{\{C\}: C \in \mathcal{C}(G)\}$ y $\mathcal{F}_{2}=\left\{\mathcal{C}_{v}: v \in V(G)\right\}$. Usar la expresión encontrada para $B_{S}$ en el Lema 4.25 y aplicar el Lema 4.24 a $C_{1}, C_{2}, \mathcal{F}_{1}, \mathcal{F}_{2}$ para obtener que

$$
B_{S}=\bigcap_{\substack{D \in \mathcal{C}(K(G)) \\ C_{1}, C_{2} \in D}} D=B_{\mathcal{F}_{1}}=B_{\mathcal{F}_{2}}=\bigcap_{C_{1}, C_{2} \in \mathcal{C}_{v}} \mathcal{C}_{v}=\bigcap_{v \in C_{1} \cap C_{2}} \mathcal{C}_{v}=\bigcap_{v \in S} \mathcal{C}_{v}=\mathcal{C}_{S}
$$

Notar que, como ya sabemos que el número de separadores minimales de vértices no supera el número de vértices en los grafos cordales, testear la condición del Teorema 4.26 es viable en términos de complejidad algorítmica.

Muchos de los resultados y demostraciones sobre grafos cordales de este capítulo suponen implícitamente que los grafos no son completos, siendo en caso contrario la familia de separadores minimales de vértices vacía. Sin embargo, es fácil ver que los teoremas y proposiciones se mantienen verdaderos aun para el caso de grafos completos.

Aunque el Teorema 4.26 fue descubierto gracias a la teoría sobre bases, si reexaminamos el Teorema 4.8 se revelará que este resultado también podría haber sido usado para dar una prueba simple del teorema 4.26. Aquí se muestra cómo: 
Teorema 4.27. Sea $G$ un grafo cordal. Son equivalentes:

1. Existen $S \in \mathcal{S}(G)$ y $C_{1}, C_{2} \in \mathcal{C}(G)$ tales que $C_{1} \cap C_{2} \subsetneq S$ y, para todo $C \in \mathcal{C}(G), C \cap S \neq \emptyset$ implica que $C \cap C_{1} \neq \emptyset$ y $C \cap C_{2} \neq \emptyset$.

2. Existe $S \in \mathcal{S}(G)$ tal que $B_{S} \neq \mathcal{C}_{S}$.

Demostración. Supongamos que 1. es verdadera y sean $C_{1}, C_{2}, S$ con las características allí mencionadas. Probaremos que $B_{S} \neq \mathcal{C}_{S}$.

Como $C_{1} \cap C_{2} \subsetneq S$, es imposible que $S \subseteq C_{1}$ y que $S \subseteq C_{2}$ simultáneamente pues, en caso contrario, $S \subseteq C_{1} \cap C_{2}$. Supongamos sin pérdida de generalidad que $S$ no está contenido en $C_{1}$. Entonces, $C_{1} \notin \mathcal{C}_{S}$ pero, por la hipótesis, $C_{1} \in B_{S}$. Por lo tanto, $B_{S} \neq \mathcal{C}_{S}$.

Recíprocamente, supongamos que 2. es verdadera y tomemos $S \in \mathcal{S}(G)$ tal que $B_{S} \neq \mathcal{C}_{S}$. Como $\mathcal{C}_{S} \subseteq B_{S}$, existe $D \in \mathcal{C}(G)$ tal que $D \in B_{S}$ y $D \notin \mathcal{C}_{S}$.

Sean $T$ un árbol clique de $G$ y $C_{3}, C_{4}$ tales que $C_{3} C_{4} \in E(T)$ y $C_{3} \cap C_{4}=S$. Entonces, $C_{4} \in T\left[C_{3}, D\right]$ o $C_{3} \in T\left[C_{4}, D\right]$. Supongamos sin pérdida de generalidad que $C_{4} \in T\left[C_{3}, D\right]$. Deducimos del Teorema 1.5 que $C_{3} \cap D \subseteq C_{3} \cap C_{4}=S$. Si $C_{3} \cap D=S$, entonces $S \subseteq D$, lo cual contradice que $D \notin \mathcal{C}_{S}$. Por lo tanto, $C_{3} \cap D \subsetneq S$.

Si $C$ es un clique de $G$ tal que $C \cap S \neq \emptyset$, entonces $C \cap C_{3} \neq \emptyset$ porque $S \subseteq C_{3}$, y $C \cap D \neq \emptyset$ porque $D \in B_{S}$. Por lo tanto, podemos fijar $C_{1}=C_{3}$ y $C_{2}=D$ para verificar que 1 . es verdadera.

Como ejemplo del Teorema 4.26, consideremos los dos grafos de la Figura 4.7.
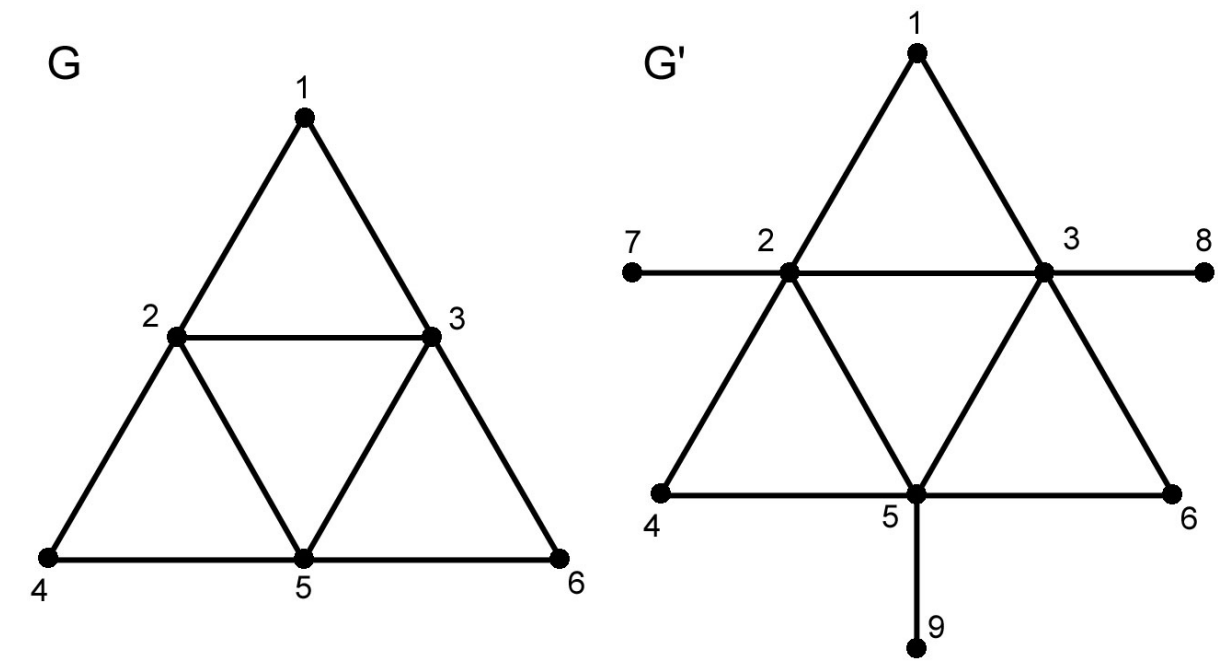

Figura 4.7: Todo árbol compatible con $K\left(G^{\prime}\right)$ es un árbol clique de $G^{\prime}$. No se puede decir lo mismo acerca de $G$.

$K(G)$ es igual al grafo completo de cuatro vértices, lo cual implica que todo árbol generador de $K(G)$ es un árbol compatible. Sin embargo, sabemos que los soles poseen solamente un árbol clique. Por lo tanto, $G$ no es básicamente cordal. Para concluir lo mismo a través del Teorema 4.26, notar que $\mathcal{S}(G)=\{\{2,3\},\{2,5\},\{3,5\}\}$ y $\mathcal{C}(G)=\{\{1,2,3\},\{2,3,5\},\{2,4,5\},\{3,5,6\}\}$. La comparación entre los conjuntos $B_{S}$ y $\mathcal{C}_{S}$, para cada $S \in \mathcal{S}(G)$, aparece en la siguiente tabla: 


\begin{tabular}{|c|c|c|c|}
\hline $\mathbf{S}$ & $B_{S}$ & $\mathcal{C}_{S}$ & $B_{S}=\mathcal{C}_{S}$ \\
\hline$\{2,3\}$ & $\mathcal{C}(G)$ & $\{\{1,2,3\},\{2,3,5\}\}$ & $\times$ \\
\hline$\{2,5\}$ & $\mathcal{C}(G)$ & $\{\{2,3,5\},\{2,4,5\}\}$ & $\times$ \\
\hline$\{3,5\}$ & $\mathcal{C}(G)$ & $\{\{2,3,5\},\{3,5,6\}\}$ & $\times$ \\
\hline
\end{tabular}

Pero si agregamos tres vértices de grado uno a $G$ para obtener el grafo $G^{\prime}$, el resultado es diferente. Esta vez, $\mathcal{C}\left(G^{\prime}\right)=\mathcal{C}(G) \cup\{\{2,7\},\{3,8\},\{5,9\}\}$ y $\mathcal{S}\left(G^{\prime}\right)=\mathcal{S}(G) \cup\{\{2\},\{3\},\{5\}\}$. La tabla correspondiente es:

\begin{tabular}{|c|c|c|c|}
\hline $\mathbf{S}$ & $B_{S}$ & $\mathcal{C}_{S}$ & $B_{S}=\mathcal{C}_{S}$ \\
\hline$\{2,3\}$ & $\{\{1,2,3\},\{2,3,5\}\}$ & $\{\{1,2,3\},\{2,3,5\}\}$ & $\checkmark$ \\
\hline$\{2,5\}$ & $\{\{2,3,5\},\{2,4,5\}\}$ & $\{\{2,3,5\},\{2,4,5\}\}$ & $\checkmark$ \\
\hline$\{3,5\}$ & $\{\{2,3,5\},\{3,5,6\}\}$ & $\{\{2,3,5\},\{3,5,6\}\}$ & $\checkmark$ \\
\hline$\{2\}$ & $\{\{1,2,3\},\{2,7\},\{2,3,5\},\{2,4,5\}\}$ & $\{\{1,2,3\},\{2,7\},\{2,3,5\},\{2,4,5\}\}$ & $\checkmark$ \\
\hline$\{3\}$ & $\{\{1,2,3\},\{3,8\},\{2,3,5\},\{3,5,6\}\}$ & $\{\{1,2,3\},\{3,8\},\{2,3,5\},\{3,5,6\}\}$ & $\checkmark$ \\
\hline$\{5\}$ & $\{\{2,4,5\},\{5,9\},\{2,3,5\},\{3,5,6\}\}$ & $\{\{2,4,5\},\{5,9\},\{2,3,5\},\{3,5,6\}\}$ & $\checkmark$ \\
\hline
\end{tabular}

Esto muestra claramente que la clase de grafos básicamente cordales no es hereditaria. De hecho, una construcción como la que se utilizó para obtener al grafo $G^{\prime}$ nos permite mostrar que todo grafo cordal es el subgrafo inducido de algún grafo básicamente cordal.

Proposición 4.28. Sean $G$ un grafo cordal y $V^{\prime}$ el conjunto de los vértices de $G$ que no son simpliciales. Sea $G^{\prime}$ el grafo construido a partir de $G$ agregando, para cada $v \in V^{\prime}$, un vértice $v^{*}$ y la arista vv*. Entonces, $G^{\prime}$ es básicamente cordal.

Demostración. Si $V^{\prime}=\emptyset$, entonces $G$ es completo, $G=G^{\prime}$ y es fácil verificar que $G$ es básicamente cordal. Si $V^{\prime} \neq \emptyset$, entonces $\mathcal{S}\left(G^{\prime}\right)=\mathcal{S}(G) \cup\left\{\{v\}: v \in V^{\prime}\right\}$. Probemos ahora que, para todo $S \in \mathcal{S}\left(G^{\prime}\right), B_{S}=\mathcal{C}_{S}$, siendo los dos conjuntos calculados con respecto a $G^{\prime}$. Sean $S \in \mathcal{S}\left(G^{\prime}\right)$, $C \in B_{S}$ y $v \in S$. Al ser $v$ el vértice de algún separador minimal de vértices, $v$ no es simplicial y, por lo tanto, $v \in V^{\prime}$. Luego, $\left\{v, v^{*}\right\}$ es un clique de $G^{\prime}$ que interseca a $S$. Como $C \in B_{S}$, se tiene que $C \cap\left\{v, v^{*}\right\} \neq \emptyset$ y, como consecuencia, que $v \in C$. Podemos concluir de este razonamiento que $S \subseteq C$, es decir, $C \in \mathcal{C}_{S}$. Por lo tanto, $B_{S} \subseteq \mathcal{C}_{S}$. Debido a las definiciones, la inclusión $\mathcal{C}_{S} \subseteq B_{S}$ siempre es verdadera. Luego, $B_{S}=\mathcal{C}_{S}$. Por el Teorema 4.26, $G^{\prime}$ es básicamente cordal.

Finalizamos esta sección enunciando una propiedad que relaciona a los grafos básicamente cordales con conceptos métricos.

Proposición 4.29. Sea $G$ un grafo cordal tal que diam $(G) \leq 2$. Entonces, $G$ es básicamente cordal si y sólo si todo vértice de $G$ es universal o simplicial.

Demostración. Supongamos que todo vértice de $G$ es universal o simplicial. Si $G$ es completo, entonces es consecuencia inmediata que $G$ es básicamente cordal. Si $G$ no es completo, entonces la intersección de cualesquiera dos cliques distintos de $G$ es igual al conjunto de vértices universales de $G$. Por lo tanto, por el Teorema 4.4, el conjunto de vértices universales de $G$ es el único separador minimal de vértices de $G$, llamémoslo $S$. Se ve fácilmente que $B_{S}=\mathcal{C}_{S}=\mathcal{C}(G)$. Luego, por el Teorema 4.26, $G$ es básicamente cordal.

Recíprocamente, supongamos que $G$ es básicamente cordal. Veamos que $K(G)$ es completo. Esto es evidente en el caso en que $G$ es completo.

En caso contrario, sean $C_{1}$ y $C_{2}$ dos cliques distintos de $G$ cualesquiera. Probaremos que $C_{1} \cap C_{2} \neq \emptyset$. Sea $T$ un árbol clique de $G$. Si $C_{1}$ y $C_{2}$ son adyacentes en $T$, entonces es claro 
que no son disjuntos. Si no son adyacentes en $T$, sea $C \in T\left(C_{1}, C_{2}\right)$. Sean también $C_{3}$ una hoja de $T$ tal que $C_{1} \in T\left[C, C_{3}\right]$ y $C_{4}$ otra hoja tal que $C_{2} \in T\left[C, C_{4}\right]$. Entonces, $C_{3}$ y $C_{4}$ son cliques simpliciales de $G$. Sean $v$ y $w$ vértices simpliciales en $C_{3}$ y $C_{4}$, respectivamente. Como $\operatorname{diam}(G) \leq 2, N[v] \cap N[w] \neq \emptyset$, es decir, $C_{3} \cap C_{4} \neq \emptyset$. Por construcción, $\left\{C_{1}, C_{2}\right\} \subseteq T\left[C_{3}, C_{4}\right] \mathrm{y}$, por la Proposición 1.5, $C_{3} \cap C_{4} \subseteq C_{1} \cap C_{2}$. Luego, $C_{1} \cap C_{2} \neq \emptyset$.

Por consiguiente, $K(G)$ es un grafo completo. Resulta así que todo árbol generador de $K(G)$ es un árbol compatible y, por ende, por ser $G$ básicamente cordal, un árbol clique de $G$.

Se deduce fácilmente de lo último que los miembros de $\mathcal{S C}(G)$ son $\mathcal{C}(G)$ y todos sus subconjuntos unitarios.

Sea $v$ un vértice de $G$. Luego, $\mathcal{C}_{v} \in \mathcal{S C}(G)$. Si $\mathcal{C}_{v}=\mathcal{C}(G)$, entonces $v$ es universal. Si $\mathcal{C}_{v}$ es un conjunto unitario, entonces $v$ es simplicial, con lo cual queda concluida la demostración.

\subsection{Determinando posibles conjuntos de hojas para los árboles compatibles de un grafo dualmente cordal}

Esta sección corta está pensada como una aplicación de la anterior. Nuevamente, la idea será aprovechar el saber cómo resolver ciertos problemas acerca de los árboles clique de los grafos cordales con el propósito de resolver problemas acerca de los árboles compatibles de los grafos dualmente cordales.

Dado un árbol $T$, se usará la expresión $\mathcal{L}(T)$ para referirnos al conjunto de hojas de $T$.

Como el título lo indica, el problema será el siguiente: dado un conjunto $A$ de vértices de un grafo dualmente cordal $G$, determinar si $G$ posee un árbol compatible $T$ tal que $\mathcal{L}(T)=A$.

Para orientarnos mejor en el proceso de encontrar una solución, hallemos primero una condición necesaria. Nuestro interés se centrará en grafos con al menos tres vértices, pues el problema es trivial sobre grafos con un número menor de vértices.

Proposición 4.30. Sean $G$ un grafo dualmente cordal y $T$ un árbol compatible con $G$. Si $v$ es una hoja de $T$ y $w$ es el vértice tal que vw $\in E(T)$, entonces $N[v] \subseteq N[w]$.

Demostración. Se verifica, para todo vértice $u$ en $N[v] \backslash\{v, w\}$, que $w \in T(u, v)$. Deducimos entonces del Teorema 1.15 que $w$ es adyacente a $u$. Por lo tanto, $N[v] \backslash\{v, w\} \subseteq N[w]$. Como $\{v, w\}$ también es un subconjunto de $N[w]$, se sigue que $N[v] \subseteq N[w]$.

Corolario 4.31. Sean $G$ un grafo dualmente cordal, $|V(G)| \geq 3$, y $T$ un árbol compatible con $G$. Entonces, todo vértice en $\mathcal{L}(T)$ está dominado por un vértice en $V(T) \backslash \mathcal{L}(T)$.

Demostración. Los árboles con más de dos vértices no tienen hojas adyacentes. Entonces, para todo vértice $v \in \mathcal{L}(T)$, el único vértice adyacente a $v$ en $T$ no está en $\mathcal{L}(T)$ y, por la Proposición 4.30 , domina a $v$.

Ahora que sabemos que debemos restringirnos a los vértices dominados, se pueden enunciar los resultados principales.

Teorema 4.32. Sean $G$ un grafo dualmente cordal $y A \subseteq V(G)$ un conjunto de vértices, cada uno de ellos dominado por un vértice en $V(G) \backslash A$. Sea $G^{\prime}$ el grafo construido a partir de $G$ agregando, para cada $v \in A$, un vértice $v^{*}$ y la arista vv*. Entonces, $G^{\prime}$ es dualmente cordal. Más aun, existe un árbol $T$ compatible con $G$ tal que $\mathcal{L}(T)=A$ si y sólo si existe un árbol $T^{\prime}$ compatible con $G^{\prime}$ tal que $\mathcal{L}\left(T^{\prime}\right)=A^{*}:=\left\{v^{*}, v \in A\right\}$. 
Demostración. Sea $T$ un árbol compatible con $G$. Entonces, el árbol $T^{\prime}$ tal que $V\left(T^{\prime}\right)=V(G) \cup$ $A^{*}$ y $E\left(T^{\prime}\right)=E(T) \cup\left\{v v^{*}, v \in A\right\}$ es compatible con $G^{\prime}$, por lo que este grafo es dualmente cordal. Además, si $\mathcal{L}(T)=A, \mathcal{L}\left(T^{\prime}\right)=A^{*}$.

Recíprocamente, supongamos que existe un árbol $T^{\prime}$ compatible con $G^{\prime}$ tal que $\mathcal{L}\left(T^{\prime}\right)=A^{*}$, y sea $T_{0}=T^{\prime}-A^{*}$. Elijamos a $T^{\prime}$ de manera que $\left|\mathcal{L}\left(T_{0}\right)\right|$ sea máximo. Como $\mathcal{C}(G) \subseteq \mathcal{C}\left(G^{\prime}\right)$, todo clique de $G$ induce un subárbol de $T^{\prime}$ y, por ende, de $T_{0}$. Entonces, $T_{0}$ es compatible con $G$. Demostremos ahora que $\mathcal{L}\left(T_{0}\right)=A$.

Es inmediato que $\mathcal{L}\left(T_{0}\right) \subseteq A$ pues, en caso contrario, cualquier vértice en $\mathcal{L}\left(T_{0}\right) \backslash A$ también sería una hoja de $T^{\prime}$, lo cual es una contradicción.

Supongamos que $\mathcal{L}\left(T_{0}\right) \neq A$. Tomemos un vértice $u \in A \backslash \mathcal{L}\left(T_{0}\right)$ y sean $w$ un vértice en $V(G) \backslash A$ que domina a $u$ y $w^{\prime}$ el vértice adyacente a $u$ en $T_{0}\langle u, w\rangle$.

Por el Teorema 4.3, si, por cada vértice $x$ diferente a $w^{\prime}$ y adyacente a $u$ en $T_{0}$, agregamos la arista $w x$ a $T_{0}$ y le quitamos la arista $u x$, obtenemos un nuevo árbol $T_{1}$ compatible con $G$ tal que el grado de $w$ es más grande en $T_{1}$ que en $T_{0}, u$ es una hoja de $T_{1}$ y todos los demás vértices tienen el mismo grado en $T_{0}$ y en $T_{1}$. Entonces, $\mathcal{L}\left(T_{1}\right)=\mathcal{L}\left(T_{0}\right) \cup\{u\}$, contradiciendo la forma en que $T_{0}$ fue elegido.

Luego, $\mathcal{L}\left(T_{0}\right)=A$.

Se define el leafage de un grafo cordal $G$ como el mínimo número de hojas de un árbol clique de $G$. Similarmente, si $G$ es dualmente cordal, se define el leafage dual de $G$ como el mínimo número de hojas de un árbol compatible de $G$. Entonces,

Teorema 4.33. Sea $G$ un grafo dualmente cordal y $H$ un grafo básicamente cordal tal que $K(H)=G$. Entonces, el leafage dual de $G$ es igual al leafage de $H$.

Demostración. Los árboles compatibles de $G$ son exactamente los árboles clique de $H$. Por lo tanto, ambos mínimos coinciden.

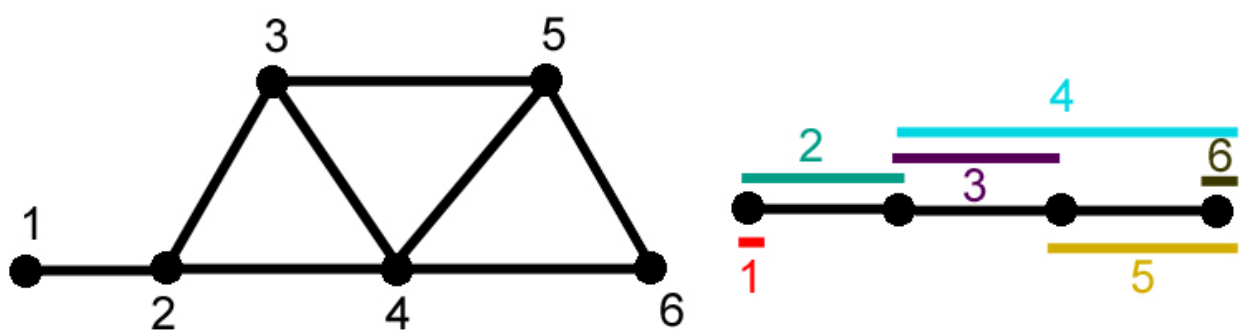

Figura 4.8: Un grafo cordal cuyo leafage es igual a 2.

Con respecto a calcular el leafage de un grafo cordal, se conoce un algoritmo polinomial para hacerlo [15].

Notar que un grafo como $H$ también puede ser obtenido en tiempo polinomial. Por ejemplo, podemos elegir, por cada arista $u v$ de $G$, un clique que contenga a $\{u, v\}$. La colección de esos cliques unida a $\{\{v\}: v \in V(G)\}$ da lugar a una familia $\mathcal{F}$ tal que $|\mathcal{F}| \leq|V(G)|+|E(G)|$. Por el Teorema $4.15, L(\mathcal{F})$ es básicamente cordal y $K(L(\mathcal{F}))=G$. Entonces, podemos hacer que $H$ sea igual a $L(\mathcal{F})$.

Por lo tanto, el leafage dual de un grafo dualmente cordal puede ser calculado en tiempo polinomial.

Ahora encontraremos la relación entre el tema de esta sección y el concepto de leafage. 
Teorema 4.34. Sean $G, G^{\prime}$ y A los mismos que en el Teorema 4.32. Sea también $H^{\prime}$ un grafo básicamente cordal tal que $K\left(H^{\prime}\right)=G^{\prime}$. Entonces, existe un árbol $T$ compatible con $G$ tal que $\mathcal{L}(T)=A$ si y sólo si el leafage de $H^{\prime}$ es igual a $|A|$.

Demostración. Por el Teorema 4.32 y el hecho de que $A^{*}$ está contenido en el conjunto de hojas de todo árbol compatible con $G^{\prime}$, existe un árbol $T$ compatible con $G$ tal que $\mathcal{L}(T)=A$ si y sólo si el leafage dual de $G^{\prime}$ es igual a $|A|$. Se concluye la prueba al notar que, por el Teorema 4.33, el leafage dual de $G^{\prime}$ es igual al leafage de $H^{\prime}$.

El algoritmo en [15] no sólo calcula el leafage de un grafo cordal, sino que también devuelve un árbol clique cuyo número de hojas es igual al leafage del grafo. Sea $T^{\prime}$ el árbol clique obtenido cuando el algoritmo es aplicado a $H^{\prime}$. Si el leafage de $H^{\prime}$ es igual a $|A|$, entonces $T^{\prime}$ es un árbol compatible con $G^{\prime}$ tal que $\mathcal{L}\left(T^{\prime}\right)=A^{*}$. Como en la demostración del Teorema 4.32, sea $T_{0}=T^{\prime}-A^{*}$. Luego, $T_{0}$ es compatible con $G$ y $\mathcal{L}\left(T_{0}\right) \subseteq A$. Si $\mathcal{L}\left(T_{0}\right) \neq A$, entonces se puede aplicar el procedimiento explicado al final de la demostración del Teorema 4.32 para incrementar el número de hojas hasta que el conjunto de hojas sea $A$. 


\section{Capítulo 5}

\section{Cómo detectar si una familia de árboles es la familia de árboles clique de un grafo cordal}

Se han considerado hasta el momento algunos problemas concernientes a los árboles clique tales como el de hallar uno de ellos y calcular el leafage. Con respecto a hallar un sólo árbol clique, sabemos a través del Teorema 4.2 que se puede hacer eficientemente. Si se quisieran hallar absolutamente todos los árboles clique de un grafo cordal, podría ocurrir que estos fueran demasiados. Dado que con un número fijo de vértices se puede obtener una cantidad exponencial de árboles, podría ser éste también el caso del número de árboles clique de un grafo cordal. No obstante, se puede encontrar un patrón en todos los árboles clique que permite diseñar un método para construirlos y existen algoritmos de tiempo polinomial para contarlos [24].

Aparentemente, el problema inverso no había sido todavía estudiado, dándosele aquí un alto grado de importancia. Para ser más claros, tomemos una familia $\mathcal{T}$ de árboles, todos con conjunto $V$ de vértices, siendo nuestro objetivo encontrar, en caso de que lo hubiera, un grafo cordal $G$ tal que $\tau(G)=\mathcal{T}$. En el presente capítulo, se determinará si un grafo así existe a través de una serie de condiciones necesarias y suficientes que pueden ser testeadas en tiempo polinomial con respecto a $|\mathcal{T}|$ y a $|V|$. Las propiedades estructurales de los árboles clique y de los conjuntos que inducen subárboles en ellos serán nuevamente aprovechadas.

Será posible también caracterizar a todos los grafos cordales que tengan a $\mathcal{T}$ como familia de árboles clique y consideraremos una versión similar del problema centrada en la identificación de árboles compatibles.

\subsection{Un método de detección por conteo}

Será preciso desarrollar una fórmula para el número total de árboles clique de un grafo cordal para encontrar las primeras condiciones necesarias y suficientes. La fórmula que se mostrará (Teorema 5.4) ya era conocida, pero será útil llegar a ella usando los conceptos del capítulo anterior.

Recordemos que una relación $\mathcal{R}$ sobre un conjunto $A$ es de equivalencia si es reflexiva, simétrica y transitiva. Un subconjunto $X$ de $A$ es una clase de equivalencia si todo par de elementos de $X$ está en la relación y $X$ es maximal en este sentido. Estas dos definiciones implican que dos clases de equivalencia distintas cualesquiera son disjuntas y que, para todo $a$ y para todo $b$ en $A, a$ y $b$ están relacionados si y sólo si ambos se encuentran en la misma clase de equivalencia. El conjunto cociente de $\mathcal{R}$, o $A / \mathcal{R}$, es el conjunto de todas las clases de 
equivalencia de $\mathcal{R}$.

Sean $G$ un grafo cordal y $S \in \mathcal{S}(G)$. Se define a $\mathcal{R}_{S}^{G}$ como la relación en $\mathcal{C}_{S}$ tal que $C_{1}$ y $C_{2}$ están relacionados si y sólo si ambos intersecan la misma componente conexa de $G-S$. Ésta es claramente una relación de equivalencia y hay una clase de equivalencia por cada componente conexa de $G-S$ que contiene a un vértice que es adyacente a todos los de $S$. El resultado que necesitamos conocer acerca de esta relación es el siguiente:

Proposición 5.1. Sean $G$ un grafo cordal y $S \in \mathcal{S}(G)$. Entonces, toda clase de equivalencia de $\mathcal{R}_{S}^{G}$ está en $\mathcal{S C}(G)$.

Demostración. Sean $X$ una clase de equivalencia de $\mathcal{R}_{S}^{G}$ y $G[A]$ la componente conexa de $G-S$ que es intersecada por los miembros de $X$.

Sea $C$ un clique de $G[A \cup S]$. Si $C \cap A=\emptyset$, entonces $C \subseteq S$. Pero $S$ no es un conjunto completo maximal de $G[A \cup S]$ porque está contenido en cada miembro de $X$. Se infiere de esta contradicción que $C \cap A \neq \emptyset$. Supongamos que $C$ no es un clique de $G$. Entonces, existe un vértice $v \in V(G) \backslash(A \cup S)$ que es adyacente a todos los vértices de $C$. De este modo, $v$ es adyacente a un vértice de $A$, lo cual es absurdo. Luego, $C$ es un clique de $G$.

Concluimos del párrafo anterior que $\mathcal{C}(G[A \cup S]) \subseteq \mathcal{C}(G)$. Probemos ahora que $\mathcal{C}(G[A \cup S]) \in$ $\mathcal{S C}(G)$. $T_{1}$.

$G[A \cup S]$ es un grafo cordal. Podemos tomar entonces un árbol clique de $G[A \cup S]$, llamémoslo

Sea $C C^{\prime}$ una arista de $T_{1}$. Necesitamos demostrar que $\mathcal{C}_{C \cap C^{\prime}}$, calculado con respecto a $G$, es un subconjunto de $\mathcal{C}(G[A \cup S])$. Supongamos por el contrario que $C^{\prime \prime}$ es un clique de $G$ tal que $C^{\prime \prime} \in \mathcal{C}_{C \cap C^{\prime}} \backslash \mathcal{C}(G[A \cup S])$. Entonces, $C \cap C^{\prime} \subseteq C \cap C^{\prime} \cap C^{\prime \prime} \subseteq S$. Por el Teorema 4.5, $C$ y $C^{\prime}$ forman un par separador de $G[A \cup S]$. Luego, $C \backslash C^{\prime}$ y $C^{\prime} \backslash C$ están contenidos en diferentes componentes conexas de $G[A \cup S]-C \cap C^{\prime}$. Sin embargo, $G[A \cup S]-C \cap C^{\prime}$ es un grafo conexo porque $G[A]$ y $G\left[S \backslash\left(C \cap C^{\prime}\right)\right]$ son conexos y, si $S \backslash\left(C \cap C^{\prime}\right) \neq \emptyset$, existe una arista de $G$ tal que uno de sus extremos está en $A$ y el otro está en $S \backslash\left(C \cap C^{\prime}\right)$. Esto nos da una contradicción.

Por lo tanto, $\mathcal{C}_{C \cap C^{\prime}} \subseteq \mathcal{C}(G[A \cup S])$. Usemos esta inclusión para deducir que $\mathcal{C}(G[A \cup S])=$ $\bigcup_{C C^{\prime} \in E\left(T_{1}\right)}^{c} \mathcal{C}_{C \cap C^{\prime}}$. Por consiguiente, $\mathcal{C}(G[A \cup S]) \in \mathcal{S C}(G)$.

Como $X=\mathcal{C}_{S} \cap \mathcal{C}(G[A \cup S]), X \in \mathcal{S C}(G)$.

Dado un árbol clique $T$ de $G$, definamos ahora a $H_{T, S}$ como el grafo cuyo conjunto de vértices es $\mathcal{C}_{S} / \mathcal{R}_{S}^{G}$, siendo $X_{1}$ y $X_{2}$ adyacentes si y sólo si existe una arista de $T$ con uno de sus extremos en $X_{1}$ y el otro en $X_{2}$. Entonces:

Proposición 5.2. Sean $G$ un grafo cordal, $T$ un árbol clique de $G$ y $S \in \mathcal{S}(G)$. Entonces, el grafo $H_{T, S}$ es un árbol.

Demostración. Como $\mathcal{C}_{S}$ y las clases de equivalencia de $\mathcal{R}_{S}^{G}$ inducen subárboles de $T$, el número de aristas de $H_{T, S}$ es igual a $\left|V\left(H_{T, S}\right)\right|-1$. Por la misma razón, $H_{T, S}$ es conexo. Luego, $H_{T, S}$ es un árbol.

Este resultado motiva la definición de aristas $S$-admisibles. Sea $E^{\prime}$ un conjunto de aristas de $K(G)$ tal que toda $e \in E^{\prime}$ tiene sus extremos en diferentes clases de equivalencia de $\mathcal{R}_{S}^{G}$. Definamos a $H_{E^{\prime}}$ como el grafo tal que $V\left(H_{E^{\prime}}\right)=\mathcal{C}_{S} / \mathcal{R}_{S}^{G}$ y, para cada $e \in E^{\prime}$, existe una arista en $H_{E^{\prime}}$ que conecta a las clases de equivalencia de los extremos de $e$. Se dice que $E^{\prime}$ es $S$-admisible cuando $H_{E^{\prime}}$ es un árbol.

Esta definición nos permite dar una caracterización de los árboles clique de un grafo cordal que no había sido mencionada hasta ahora. 

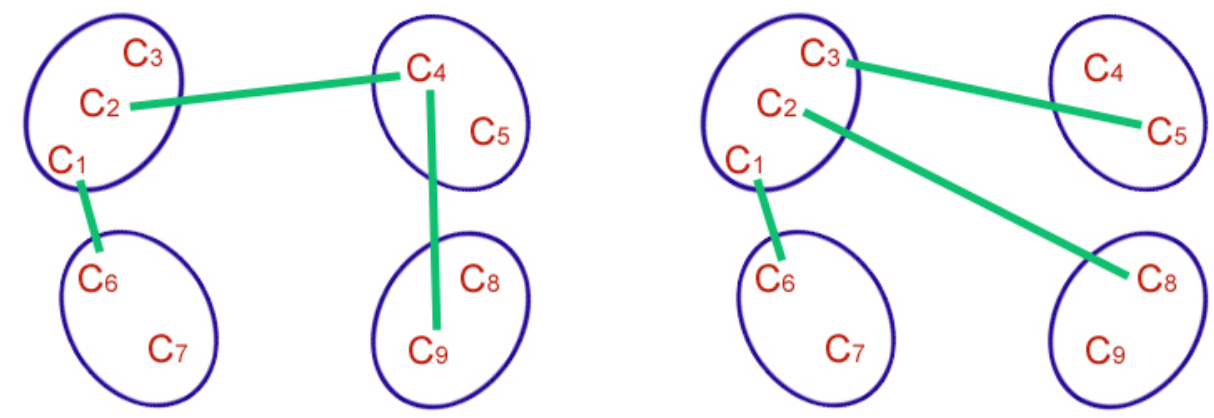

Figura 5.1: Supongamos que $G$ y $\quad S \quad \in \quad \mathcal{S}(G)$ son tales que $\mathcal{C}_{S} / \mathcal{R}_{S}^{G}=$ $\left\{\left\{C_{1}, C_{2}, C_{3}\right\},\left\{C_{4}, C_{5}\right\},\left\{C_{6}, C_{7}\right\},\left\{C_{8}, C_{9}\right\}\right\}$. Entonces, se muestran en la figura dos conjuntos de aristas $S$-admisibles.

Teorema 5.3. Sean $G$ un grafo cordal no completo, $T$ un árbol tal que $V(T)=\mathcal{C}(G)$ y $\mathcal{S}(G)=$ $\left\{S_{1}, \ldots, S_{j}\right\}$. Entonces, son equivalentes:

(a) $T$ es un árbol clique de $G$.

(b) $E(T)=\bigcup_{i=1}^{j} E_{i}$, siendo $E_{i} S_{i}$-admisible para todo $1 \leq i \leq j$.

\section{Demostración.}

$(a) \Rightarrow(b)$ : Véase la Proposición 5.2.

(b) $\Rightarrow(a)$ : Sean $T_{0}$ un árbol clique de $G$ y $A_{1}$ el conjunto de todas las aristas de $T_{0}$ tales que la intersección de los extremos de cada una de ellas es igual a $S_{1}$. Definamos $T_{1}=T_{0}-A_{1}+E_{1}$. No es difícil verificar que $T_{1}$ es un árbol. Ahora probaremos que $T_{1}$ es un árbol clique de $G$.

Supongamos que $v \in V(G)$ es tal que $\mathcal{C}_{v}$ no induce un subárbol de $T_{1}$. Esto implica que la remoción de alguna arista en $A_{1}$ desconecta a $T_{0}\left[\mathcal{C}_{v}\right]$ y que los extremos de esa arista quedan en diferentes componentes conexas de $T_{1}\left[\mathcal{C}_{v}\right]$. Como la intersección de esos cliques es igual a $S_{1}$ y $v$ está en ambos, $v \in S_{1}$.

Por otro lado, no es difícil ver que, por construcción, $T_{1}\left[\mathcal{C}_{S_{1}}\right]$ es conexo. Ya que $\mathcal{C}_{S_{1}} \subseteq \mathcal{C}_{v}$, todos los cliques en $\mathcal{C}_{S_{1}}$ están en la misma componente conexa de $T_{1}\left[\mathcal{C}_{v}\right]$, lo cual produce un absurdo.

Concluimos de este razonamiento que $T_{1}$ es un árbol clique de $G$.

Para todo $1 \leq i \leq j$, definamos recursivamente a $A_{i}$ como el conjunto de todas las aristas de $T_{i-1}$ tales que la intersección de los extremos de cada una de ellas es igual a $S_{i}$, y $T_{i}=$ $T_{i-1}-A_{i}+E_{i}$. Por los mismos razonamientos que antes, $T_{i}$ es un árbol clique de $G$ para todo $1 \leq i \leq j$. Además, $T_{j}=T$. Luego, $T$ es un árbol clique.

Por todo lo visto, ya es posible dar con la fórmula del número total de árboles clique de un grafo cordal:

Teorema 5.4. Sea $G$ un grafo cordal. Entonces, el número de árboles clique de $G$ es igual a

$$
\prod_{S \in \mathcal{S}(G)}\left[\left(\prod_{X \in \mathcal{C}_{S} / R_{S}^{G}}|X|\right)\left|\mathcal{C}_{S}\right|^{\left|\mathcal{C}_{S} / R_{S}^{G}\right|-2}\right] \text {. }
$$


Demostración. Es claro, debido al Teorema 5.3, que el número total de árboles clique de $G$ es igual a $\prod_{S \in \mathcal{S}(G)} N_{S}$, siendo $N_{S}$ el número total de conjuntos de aristas $S$-admisibles que hay, $S \in \mathcal{S}(G)$. Encontremos una fórmula para $N_{S}$.

Sean $\mathcal{C}_{S} / \mathcal{R}_{S}^{G}=\left\{X_{1}, \ldots, X_{k}\right\}$ y $T$ un árbol clique de $G$.

Si sabemos que, para todo $1 \leq i \leq k$, el grado de $X_{i}$ en $H_{T, S}$ es $d_{i}$, entonces, por la prueba de la fórmula de Cayley dada por Prüfer [22], existen $\left(\begin{array}{c}k-2 \\ d_{1}-1 \ldots d_{k}-1\end{array}\right)$ árboles posibles a los que $H_{T, S}$ puede ser igual.

Si sabemos ahora con exactitud cuál es $H_{T, S}$, entonces el número de conjuntos de aristas $S$-admisibles que $T$ puede llegar a tener es igual a $\prod_{i=1}^{k}\left|X_{i}\right|^{d_{i}}$.

$\mathrm{Al}$ ser la suma de los grados de los vértices de $\stackrel{i=1}{H}_{T, S}$ igual a $2 k-2$, tenemos que

$$
\begin{aligned}
& N_{S}=\sum_{d_{1}+\ldots+d_{k}=2 k-2}\left[\left(\begin{array}{c}
k-2 \\
d_{1}-1 \ldots d_{k}-1
\end{array}\right) \prod_{i=1}^{k}\left|X_{i}\right|^{d_{i}}\right]= \\
& \left(\prod_{i=1}^{k}\left|X_{i}\right| \sum_{d_{1}+\ldots+d_{k}=2 k-2}\left[\left(\begin{array}{c}
k-2 \\
d_{1}-1 \ldots d_{k}-1
\end{array}\right) \prod_{i=1}^{k}\left|X_{i}\right|^{d_{i}-1}\right]=\right. \\
& \left(\prod_{i=1}^{k}\left|X_{i}\right|\right) \sum_{e_{1}+\ldots+e_{k}=k-2}\left[\left(\begin{array}{c}
k-2 \\
e_{1} \ldots e_{k}
\end{array}\right) \prod_{i=1}^{k}\left|X_{i}\right|^{e_{i}}\right]=\left(\prod_{i=1}^{k}\left|X_{i}\right|\right)\left(\sum_{i=1}^{k}\left|X_{i}\right|\right)^{k-2} \\
& =\left(\prod_{i=1}^{k}\left|X_{i}\right|\right)\left|\mathcal{C}_{S}\right|^{k-2}
\end{aligned}
$$

Si queremos que los conjuntos, grafos y relaciones que fueron presentados en este capítulo puedan ser aplicados para obtener un método de detección de familias de árboles clique de los grafos cordales, entonces deberemos ser capaces de identificarlos con sólo examinar a los árboles clique, sin necesidad de mirar al grafo. Los siguientes resultados indican cómo eso es posible.

Dada una familia $\mathcal{T}$ de árboles con conjunto $V$ de vértices, hay dos grafos asociados a ella que serán importantes en esta sección y en la que sigue. El grafo $H_{\mathcal{T}}$ tiene conjunto de vértices igual a $\mathcal{T}$, siendo $T$ y $T^{\prime}, T \neq T^{\prime}$, adyacentes en $H_{\mathcal{T}}$ si y sólo si existen aristas $e$ y $e^{\prime}$ tales que $T^{\prime}=T-e+e^{\prime}$. Sea $E(\mathcal{T})$ el conjunto de aristas que están en al menos un árbol de $\mathcal{T}$. Denotemos por $H_{\mathcal{T}}^{*}$ al grafo tal que $V\left(H_{\mathcal{T}}^{*}\right)=E(\mathcal{T})$, de modo que $e, e^{\prime} \in E(\mathcal{T}), e \neq e^{\prime}$, son adyacentes si y sólo si existe $T \in \mathcal{T}$ tal que $T-e+e^{\prime} \in \mathcal{T}$. Por cada componente conexa $H^{\prime}$ de $H_{\mathcal{T}}^{*}$, definamos $P\left(H^{\prime}\right)=\bigcup_{u v \in V\left(H^{\prime}\right)}\{u, v\}$.

Entonces tenemos que:

Proposición 5.5. Sea $G$ un grafo cordal. Entonces, $H_{\tau(G)}$ es conexo.

Demostración. Aplicar el Teorema 4.2 y la Proposición 4.7.

Procedamos ahora a caracterizar al grafo $H_{\tau(G)}^{*}$ : 

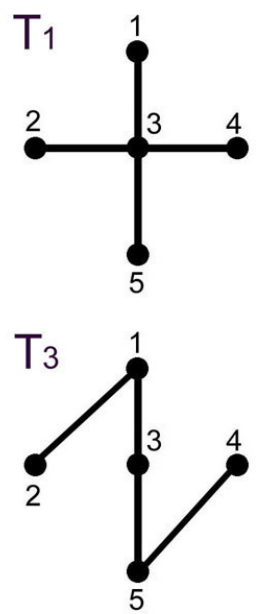

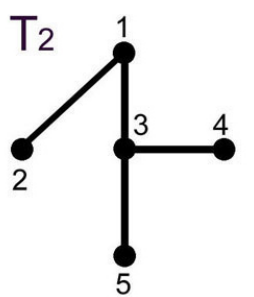

$\mathrm{T}_{4}$

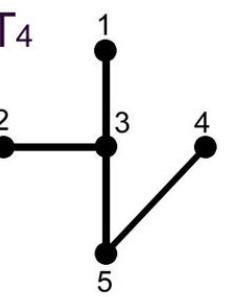

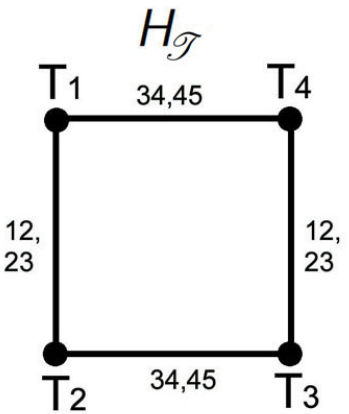

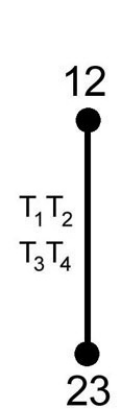

Figura 5.2: Una familia $\mathcal{T}$ de árboles y los grafos $H_{\mathcal{T}}$ y $H_{\mathcal{T}}^{*}$. Cada arista de $H_{\mathcal{T}}$ está etiquetada con las aristas que los árboles deben intercambiar para obtener uno a partir del otro. Cada arista $e e^{\prime}$ de $H_{\mathcal{T}}^{*}$ está etiquetada con todos los pares de árboles que pueden intercambiar a $e$ y a $e^{\prime}$ para obtener a uno a partir del otro.

Proposición 5.6. Sean $G$ un grafo cordal y $C_{1} C_{2}, C_{3} C_{4}$ dos elementos diferentes de $E(\tau(G))$. Entonces, $C_{1} C_{2}$ y $C_{3} C_{4}$ son adyacentes en $H_{\tau(G)}^{*}$ si y sólo si $C_{1} \cap C_{2}=C_{3} \cap C_{4}$.

Demostración. Supongamos que $C_{1} C_{2}$ y $C_{3} C_{4}$ son adyacentes en $H_{\tau(G)}^{*}$. Sea $T$ un árbol clique de $G$ tal que $T-C_{1} C_{2}+C_{3} C_{4}$ es también un árbol clique. Entonces, $C_{1}, C_{2} \in T\left[C_{3}, C_{4}\right]$, por lo que $C_{3} \cap C_{4} \subseteq C_{1} \cap C_{2}$. Además, por el Teorema 4.2, $\left|C_{1} \cap C_{2}\right|=\left|C_{3} \cap C_{4}\right|$. Por lo tanto, $C_{1} \cap C_{2}=C_{3} \cap C_{4}$.

Recíprocamente, supongamos que $C_{1} \cap C_{2}=C_{3} \cap C_{4}$. Sean $S=C_{1} \cap C_{2}$ y $G\left[A_{1}\right], G\left[A_{2}\right], G\left[A_{3}\right]$, $G\left[A_{4}\right]$ las componentes conexas de $G-S$ que intersecan a $C_{1}, C_{2}, C_{3}, C_{4}$, respectivamente. Sea también $B$ el conjunto de vértices de las otras componentes conexas, si las hubiera. Entonces, por el Teorema $4.5, A_{1} \neq A_{2}$ y $A_{3} \neq A_{4}$. Consideremos los siguientes tres casos:

1) $A_{1}, A_{2}, A_{3}, A_{4}$ son todos diferentes: Sean $T_{1}$ un árbol clique de $G\left[A_{1} \cup B \cup S\right]$ y $T_{i}, i=2,3,4$, un árbol clique de $G\left[A_{i} \cup S\right]$. Sea $T=T_{3}+C_{1} C_{3}+T_{1}+C_{1} C_{2}+T_{2}+C_{2} C_{4}+T_{4}$. Probemos que $T$ es un árbol clique. Sea $v \in V(G)$. Si $v \notin S$, entonces $v$ está en $A_{1} \cup B, A_{2}, A_{3}$ о $A_{4}$. Si $v \in A_{1} \cup B$, entonces $T\left[\mathcal{C}_{v}\right]=T_{1}\left[\mathcal{C}_{v}\right]$, que es un subárbol. Si $v \in A_{i}, i=2,3$, 4 , entonces $T\left[\mathcal{C}_{v}\right]=T_{i}\left[\mathcal{C}_{v}\right]$, también un subárbol. Si $v \in S$, entonces $T\left[\mathcal{C}_{v}\right]$ está formado por los subárboles $T_{1}\left[\mathcal{C}_{v} \cap \mathcal{C}\left(G\left[A_{1} \cup B \cup S\right]\right)\right]$ y $T_{i}\left[\mathcal{C}_{v} \cap \mathcal{C}\left(G\left[A_{i} \cup S\right]\right)\right], i=2,3,4$, todos unidos por las aristas $C_{1} C_{3}$, $C_{1} C_{2}$ y $C_{2} C_{4}$. Por lo tanto, $T\left[\mathcal{C}_{v}\right]$ es un subárbol. Podemos concluir que $T$ es un árbol clique. Similarmente, $T-C_{1} C_{2}+C_{3} C_{4}$ también es un árbol clique. Luego, $C_{1} C_{2}$ y $C_{3} C_{4}$ son adyacentes en $H_{\tau(G)}^{*}$.

2) Dos de los conjuntos son iguales: Supongamos sin pérdida de generalidad que $A_{1}=A_{3}$. Sea $T=T_{1}+C_{1} C_{2}+T_{2}+C_{2} C_{4}+T_{4}$. Entonces, $T$ es un árbol clique de $G$ y también lo es $T-C_{1} C_{2}+C_{3} C_{4}$. Luego, $C_{1} C_{2}$ y $C_{3} C_{4}$ son adyacentes en $H_{\tau(G)}^{*}$.

3) Hay dos pares de conjuntos que son iguales: Supongamos sin pérdida de generalidad que $A_{1}=A_{3}$ y que $A_{2}=A_{4}$. Sea $T=T_{1}+C_{1} C_{2}+T_{2}$. Entonces, $T$ es un árbol clique de $G$ y también lo es $T-C_{1} C_{2}+C_{3} C_{4}$. Luego, $C_{1} C_{2}$ y $C_{3} C_{4}$ son adyacentes en $H_{\tau(G)}^{*}$.

En combinación con los Teoremas 4.4 y 4.5, se obtiene: 
Corolario 5.7. Sea $G$ un grafo cordal. Entonces, el número de componentes conexas de $H_{\tau(G)}^{*}$ es igual a $|\mathcal{S}(G)|$ y cada una de ellas es un subgrafo completo.

Estudiemos ahora a esas componentes conexas.

Proposición 5.8. Sean $G$ un grafo cordal, $C_{1} C_{2} \in E(\tau(G))$ y $C_{3}$ otro clique tal que $C_{1} \cap C_{2} \subseteq$ $C_{3}$. Entonces, $C_{1} C_{2}$ es adyacente a $C_{1} C_{3}$ o a $C_{2} C_{3}$ en $H_{\tau(G)}^{*}$.

Demostración. Sea $T$ un árbol clique de $G$ tal que $C_{1} C_{2} \in E(T)$. Entonces, $C_{1} \in T\left[C_{2}, C_{3}\right]$ o $C_{2} \in T\left[C_{1}, C_{3}\right]$. En el primer caso, $T-C_{1} C_{2}+C_{2} C_{3}$ es un árbol clique, por lo que $C_{1} C_{2}$ y $C_{2} C_{3}$ son adyacentes en $H_{\tau(G)}^{*}$. En el segundo caso, $T-C_{1} C_{2}+C_{1} C_{3}$ es un árbol clique. Luego, $C_{1} C_{2}$ y $C_{1} C_{3}$ son adyacentes en $H_{\tau(G)}^{*}$.

Proposición 5.9. Sean $G$ un grafo cordal, $C_{1} C_{2} \in E(\tau(G)), C_{1} \cap C_{2}=S$, y $H^{\prime}$ la componente conexa de $H_{\tau(G)}^{*}$ que contiene a $C_{1} C_{2}$. Entonces, $P\left(H^{\prime}\right)=\mathcal{C}_{S}$.

Demostración. Sea $C \in P\left(H^{\prime}\right)$. Tomemos un clique $C^{\prime}$ tal que $C C^{\prime} \in V\left(H^{\prime}\right)$. Entonces, por la Proposición 5.6 y el Corolario 5.7, $C \cap C^{\prime}=C_{1} \cap C_{2}=S$, y por ende $C \in \mathcal{C}_{S}$. Luego, $P\left(H^{\prime}\right) \subseteq \mathcal{C}_{S}$.

Recíprocamente, sea $C \in \mathcal{C}_{S}$. Si $C=C_{1}$ o $C=C_{2}$, entonces claramente $C \in P\left(H^{\prime}\right)$. Si no, por la Proposición 5.8, $C_{1} C_{2}$ es adyacente a $C C_{1}$ en $H_{\tau(G)}^{*}$ o $C_{1} C_{2}$ es adyacente a $C C_{2}$ en $H_{\tau(G)}^{*}$. Por lo tanto, $C C_{1} \in V\left(H^{\prime}\right)$ o $C C_{2} \in V\left(H^{\prime}\right)$. En cualquier caso, concluimos que $C \in P\left(H^{\prime}\right)$. Se desprende que $\mathcal{C}_{S} \subseteq P\left(H^{\prime}\right)$.

Luego, $P\left(H^{\prime}\right)=\mathcal{C}_{S}$.

Ahora que $\mathcal{C}_{S}$ pudo ser identificado mirando a los árboles clique, hagamos lo mismo con la relación $\mathcal{R}_{S}^{G}$.

Proposición 5.10. Sean $G$ un grafo cordal, $S \in \mathcal{S}(G)$ y $C_{1}, C_{2} \in \mathcal{C}_{S}$. Entonces, $\left(C_{1}, C_{2}\right) \in \mathcal{R}_{S}^{G}$ si y sólo si $C_{1} C_{2} \notin V\left(H^{\prime}\right)$, siendo $H^{\prime}$ la componente conexa de $H_{\tau(G)}^{*}$ tal que $P\left(H^{\prime}\right)=\mathcal{C}_{S}$.

Demostración. Supongamos que $\left(C_{1}, C_{2}\right) \in \mathcal{R}_{S}^{G}$. Si $C_{1} \cap C_{2} \neq S$, entonces $C_{1} C_{2}$ no puede ser un vértice de $H^{\prime}$.

Si $C_{1} \cap C_{2}=S$, entonces $C_{1}$ y $C_{2}$ no forman un par separador. Por lo tanto, $C_{1} C_{2}$ no es la arista de ningún árbol clique de $G$, lo cual imposibilita que sea un vértice de $H_{\tau(G)}^{*}$, y mucho menos de $H^{\prime}$.

Supongamos ahora que $\left(C_{1}, C_{2}\right) \notin \mathcal{R}_{S}^{G}$. Entonces, $C_{1}$ y $C_{2}$ forman un par separador y $C_{1} \cap$ $C_{2}=S$. Por lo primero, $C_{1} C_{2} \in E(\tau(G))$ y, por lo segundo, $C_{1} C_{2} \in V\left(H^{\prime}\right)$.

Todos los resultados previos bastan para enunciar las primeras condiciones necesarias y suficientes para que una familia de árboles sea la familia de árboles clique de un grafo cordal.

Sean $\mathcal{T}$ una familia de árboles y $H^{\prime}$ una componente conexa de $H_{\mathcal{T}}^{*}$. Se define a $\mathcal{R}_{H^{\prime}}^{\mathcal{T}}$ como la relación sobre $P\left(H^{\prime}\right)$ tal que $(u, v) \in \mathcal{R}_{H^{\prime}}^{\mathcal{T}}$ si y sólo si $u v \notin V\left(H^{\prime}\right)$. Entonces:

Teorema 5.11. Sean $\mathcal{T}$ una familia de árboles, con conjunto de vértices igual a $V$, y $\mathcal{Q}$ la familia de componentes conexas de $H_{\mathcal{T}}^{*}$. Entonces, existe un grafo cordal $G$ tal que $\tau(G)=\mathcal{T}$ si y sólo si se cumplen cada una de las siguientes condiciones:

1. Para toda componente conexa $H^{\prime}$ de $H_{\mathcal{T}}^{*}, \mathcal{R}_{H^{\prime}}^{\mathcal{T}}$ es una relación de equivalencia.

2. Para toda componente conexa $H^{\prime}$ de $H_{\mathcal{T}}^{*}$ y todo $T \in \mathcal{T}, P\left(H^{\prime}\right)$ y las clases de equivalencia de $\mathcal{R}_{H^{\prime}}^{\mathcal{T}}$ inducen subárboles de $T$. 
3. $|\mathcal{T}|=\prod_{H^{\prime} \in \mathcal{Q}}\left[\left(\prod_{X \in P\left(H^{\prime}\right) / \mathcal{R}_{H^{\prime}}^{\mathcal{T}}}|X|\right)\left|P\left(H^{\prime}\right)\right|^{\left|P\left(H^{\prime}\right) / \mathcal{R}_{H^{\prime}}^{\mathcal{T}}\right|-2}\right]$

Demostración. Supongamos que $\mathcal{T}$ es la familia de árboles clique de un grafo $G$. Entonces, la condición 1 es una consecuencia de la Proposición 5.10, la condición 2 es una consecuencia de las Proposiciones 5.9 y 5.1 y la condición 3 es una consecuencia de las proposiciones que ya se han mencionado y del Teorema 5.4.

Recíprocamente, supongamos que se verifican las tres condiciones. Sea $\mathcal{F}=\left(\bigcup_{H^{\prime} \in \mathcal{Q}} P\left(H^{\prime}\right) / \mathcal{R}_{H^{\prime}}^{\mathcal{T}}\right)$ $\bigcup\left\{P\left(H^{\prime}\right): H^{\prime} \in \mathcal{Q}\right\} \bigcup\{\{v\}: v \in V\}$ y $G=L(\mathcal{F})$. Entonces, por el Teorema 1.4 y la condición $2, G$ es un grafo cordal. Por la Proposición $4.14,|\mathcal{C}(G)|=|V|$ y cada $T \in \mathcal{T}$ se corresponde con un árbol clique de $G$.

Sea $T$ un árbol clique de $G$ y $H^{\prime}$ una componente conexa de $H_{\mathcal{T}}^{*}$. Por la forma en que $G$ fue construido, el grafo cuyo conjunto de vértices es $P\left(H^{\prime}\right) / \mathcal{R}_{H^{\prime}}^{\mathcal{T}}$ y tal que $X_{1}$ y $X_{2}$ en $P\left(H^{\prime}\right) / \mathcal{R}_{H^{\prime}}^{\mathcal{T}}$ son adyacentes si y sólo si $T$ posee una arista con uno de sus extremos en $X_{1}$ y el otro en $X_{2}$ es un árbol. Notar que una arista como la de la oración anterior debe estar en $V\left(H^{\prime}\right)$. Recíprocamente, por la condición 1, toda $e \in V\left(H^{\prime}\right)$ tiene sus extremos en diferentes clases de equivalencia de $\mathcal{R}_{H^{\prime}}^{\mathcal{T}}$.

Entonces, razonando como en la prueba del Teorema 5.4, el número de maneras posibles en las que las aristas que están en $V\left(H^{\prime}\right)$ pueden ser elegidas en el proceso de construir un árbol clique de $G$ es a lo sumo $\left(\prod_{X \in P\left(H^{\prime}\right) / \mathcal{R}_{H^{\prime}}^{\mathcal{T}}}|X|\right)\left|P\left(H^{\prime}\right)\right|^{\left|P\left(H^{\prime}\right) / \mathcal{R}_{H^{\prime}}^{\mathcal{T}}\right|-2}$.

Luego de hacer esta elección para cada $H^{\prime} \in \mathcal{Q}$, no es necesario seleccionar más aristas para obtener un árbol clique de $G$ pues, de lo contrario, el árbol clique resultante tendría más aristas que cualquier $T \in \mathcal{T}$, que también es un árbol clique de $G$, lo cual constituye una contradicción.

Por lo tanto, $|\tau(G)| \leq \prod_{H^{\prime} \in \mathcal{Q}}\left[\left(\prod_{X \in P\left(H^{\prime}\right) / \mathcal{R}_{H^{\prime}}^{\mathcal{T}}}|X|\right)\left|P\left(H^{\prime}\right)\right|^{\left|P\left(H^{\prime}\right) / \mathcal{R}_{H^{\prime}}^{\mathcal{T}}\right|-2}\right]$.

Por la condición $3,|\tau(G)| \leq|\mathcal{T}|$. Sabíamos también que $\mathcal{T} \subseteq \tau(G)$. Luego, $\tau(G)=\mathcal{T}$.

Consideremos, por ejemplo, a la familia de árboles de la Figura 5.2. En este caso, $H_{\mathcal{T}}^{*}$ posee cuatro componentes conexas. Sean $H_{1}^{\prime}$ la componente conexa con vértices 12 y $13, \mathrm{y}$ $H_{2}^{\prime}$ la componente conexa con vértices 34 y 45 . Entonces, $P\left(H_{1}^{\prime}\right)=\{1,2,3\}, P\left(H_{2}^{\prime}\right)=\{3,4,5\}$, $\mathcal{R}_{H_{1}^{\prime}}^{\mathcal{T}}=\{(1,1),(2,2),(3,3),(1,3),(3,1)\}$ y $\mathcal{R}_{H_{2}^{\prime}}^{\mathcal{T}}=\{(3,3),(4,4),(5,5),(3,5),(5,3)\}$. Por lo tanto, estas relaciones son de equivalencia, de modo que $P\left(H_{1}^{\prime}\right) / \mathcal{R}_{H_{1}^{\prime}}^{\mathcal{T}}=\{\{1,3\},\{2\}\}$ y $P\left(H_{2}^{\prime}\right) / \mathcal{R}_{H_{2}^{\prime}}^{\mathcal{T}}=$ $\{\{3,5\},\{4\}\}$. Esto nos permite ver que se satisface la condición 2. Además,

$$
\begin{gathered}
\prod_{H^{\prime} \in \mathcal{Q}}\left[\left(\prod_{X \in P\left(H^{\prime}\right) / \mathcal{R}_{H^{\prime}}^{\mathcal{T}}}|X|\right)\left|P\left(H^{\prime}\right)\right|^{\left|P\left(H^{\prime}\right) / \mathcal{R}_{H^{\prime}}^{\mathcal{T}}\right|-2}\right]= \\
{\left[(|\{1,3\}| \cdot|\{2\}|) \cdot\left|P\left(H_{1}^{\prime}\right)\right|^{\left|P\left(H_{1}^{\prime}\right) / \mathcal{R}_{H_{1}^{\prime}}^{\mathcal{T}}\right|-2}\right] \cdot\left[(|\{3,5\}| \cdot|\{4\}|) \cdot\left|P\left(H_{2}^{\prime}\right)\right|^{\left|P\left(H_{2}^{\prime}\right) / \mathcal{R}_{H_{2}^{\prime}}^{\mathcal{T}}\right|-2}\right]=} \\
{\left[(2.1) \cdot 3^{2-2}\right] \cdot\left[(2.1) \cdot 3^{2-2}\right]=2 \cdot 2=4}
\end{gathered}
$$

No hemos considerado a las otras componentes conexas porque es trivial verificar que satisfacen las condiciones 1 y 2 y que no alteran el producto. Como el resultado es igual a la cantidad de árboles de la familia, también se satisface la condición 3. Luego, los árboles de la Figura 5.2 constituyen la familia de árboles clique de algún grafo cordal. 


\subsection{Otro método de detección}

Encontraremos en esta sección nuevas condiciones necesarias y suficientes para que una familia de árboles sea la familia de árboles clique de un grafo cordal. Estas condiciones serán estructurales y no involucrarán conteo alguno. Están basadas en la idea de construir un grafo que será el potencial candidato a tener los árboles dados como árboles clique.

Una vez más, será útil encontrar más caracterizaciones de los árboles clique de un grafo cordal e identificar a los conjuntos que inducen subárboles en todos los árboles clique con simplemente examinar a éstos. Esas cosas son las que se hacen a continuación:

Proposición 5.12. Sean $G$ un grafo cordal y $\mathcal{B}$ una subfamilia generadora de $\mathcal{S C}(G)$. Entonces:

(a) $T$ es un árbol clique de $G$ si y sólo si todo miembro de $\mathcal{B}$ induce un subárbol de $T$.

(b) Sea $\mathcal{F}=\mathcal{B} \cup\{\{C\}: C \in \mathcal{C}(G)\}$. Entonces, el grafo de intersección de $\mathcal{F}$ es cordal y tiene los mismos árboles clique que $G$.

\section{Demostración.}

(a) Sea $T$ un árbol clique de $G$. Entonces, como $\mathcal{B} \subseteq \mathcal{S C}(G)$, todo miembro de $\mathcal{B}$ induce un subárbol de $T$.

Recíprocamente, supongamos que $T$ es un árbol cuyo conjunto de vértices es $\mathcal{C}(G)$ y tal que todo miembro de $\mathcal{B}$ induce un subárbol de $T$. Para cada $v \in V(G), \mathcal{C}_{v}$ es un conjunto unitario o, si no, puede ser expresado como la unión conexa de miembros de $\mathcal{B}$. Por lo tanto, $\mathcal{C}_{v}$ induce un subárbol de $T$. Luego, $T$ es un árbol clique.

(b) Sea $G^{\prime}=L(\mathcal{F})$. Puede representarse a $G^{\prime}$ como el grafo de intersección de subárboles de cualquier árbol clique de $G$, por lo que es un grafo cordal.

Por la proposición 4.14, la familia de cliques de $L(\mathcal{F})$ consiste de todos los conjuntos $D_{C}$, $C \in \mathcal{C}(G)$. Para $F \in \mathcal{F}$, el conjunto de cliques de $L(\mathcal{F})$ que contienen a $F$ es $\left\{D_{C}: C \in F\right\}$. Entonces, $\left\{\mathcal{C}_{v}\right\}_{v \in V\left(G^{\prime}\right)} \cong \mathcal{F}$. Debido a la forma en que $\mathcal{F}$ fue construida, es consecuencia de la parte $(a)$ que $T$ es un árbol clique de $G$ si y sólo si todo miembro de $\mathcal{F}$ induce un subárbol de $T$, es decir, si y sólo si $T$ es un árbol clique de $G^{\prime}$. Luego, $G$ y $G^{\prime}$ tienen los mismos árboles clique.

En este contexto, la Proposición 5.12 significa que si alguien encuentra un grafo cordal que demuestra que la respuesta a nuestro problema de decisión es afirmativa, no mostrándonos ese grafo sino una familia que genera a los conjuntos que inducen subárboles de todo árbol clique del grafo, entonces podríamos corroborarlo construyendo otro grafo. Por consiguiente, conocer una familia generadora es casi tan importante como conocer al mismo grafo cordal. Esto también nos sugiere que, dada $\mathcal{T}$, intentar deducir a partir de ella una familia generadora asociada a un grafo cordal que potencialmente tiene a $\mathcal{T}$ como familia de árboles clique sería una forma muy apropiada para encarar la cuestión. Sabemos cómo hacer esto usando la Proposición 5.9. Encontramos abajo una manera alternativa que podría llegar a ser más simple.

Proposición 5.13. Sean $G$ un grafo cordal, $S \in \mathcal{S}(G)$ y $C_{1} C_{2} \in E(\tau(G))$ tales que $C_{1} \cap C_{2}=S$. Definamos $\mathcal{T}_{G}\left[C_{1}, C_{2}\right]=\bigcup_{T \in \tau(G)} T\left[C_{1}, C_{2}\right]$. Entonces, $\mathcal{T}_{G}\left[C_{1}, C_{2}\right]=\mathcal{C}_{S}$.

Demostración. Sean $C$ un elemento cualquiera de $\mathcal{T}_{G}\left[C_{1}, C_{2}\right]$ y $T \in \mathcal{T}(G)$ tal que $C \in T\left[C_{1}, C_{2}\right]$. $\mathrm{Al}$ ser $T$ un árbol clique, $C_{1} \cap C_{2} \subseteq C$ y, por ende, $C \in \mathcal{C}_{S}$. Luego, $\mathcal{T}_{G}\left[C_{1}, C_{2}\right] \subseteq \mathcal{C}_{S}$.

Supongamos ahora que $C \in \mathcal{C}_{S}$. Si $C=C_{1}$ o $C=C_{2}$, es claro entonces que $C \in \mathcal{T}_{G}\left[C_{1}, C_{2}\right]$. En caso contrario, por la Proposición 5.8, $C_{1} C_{2}$ es adyacente a $C C_{1}$ en $H_{\tau(G)}^{*}$ o $C_{1} C_{2}$ es adyacente 
a $C C_{2}$ en $H_{\tau(G)}^{*}$. Supongamos sin pérdida de generalidad que vale lo primero. Sea $T$ un árbol clique de $G$ tal que $T-C C_{1}+C_{1} C_{2}$ también es árbol clique. Entonces, $C \in T\left[C_{1}, C_{2}\right]$, y de aquí que $C \in \mathcal{T}_{G}\left[C_{1}, C_{2}\right]$.

Luego, $\mathcal{C}_{S} \subseteq \mathcal{T}_{G}\left[C_{1}, C_{2}\right]$ y se sigue la igualdad.

Con la ayuda de las Proposiciones 5.12 y 5.13, se obtiene:

Teorema 5.14. Sean $\mathcal{T}$ una familia de árboles, todos con conjunto de vértices $V, T_{1} \in \mathcal{T}$, $\mathcal{F}=\left\{\mathcal{T}[u, v]:\right.$ uv $\left.\in E\left(T_{1}\right)\right\}$ y $\mathcal{F}^{\prime}=\mathcal{F} \cup\{\{v\}: v \in V\}$. Entonces, existe un grafo cordal $G$ tal que $\tau(G)=\mathcal{T}$ si y sólo si $L\left(\mathcal{F}^{\prime}\right)$ es cordal y $\tau\left(L\left(\mathcal{F}^{\prime}\right)\right)=\mathcal{T}$.

Demostración. Supongamos que existe un grafo cordal $G$ tal que $\tau(G)=\mathcal{T}$. Entonces, por la Proposición 5.13 y el Teorema $4.13, \mathcal{F}$ es una subfamilia generadora de $\mathcal{S C}(G)$ y, por la Proposición 5.12, $L\left(\mathcal{F}^{\prime}\right)$ es cordal y $\tau\left(L\left(\mathcal{F}^{\prime}\right)\right)=\mathcal{T}$.

La recíproca es claramente verdadera. Basta con hacer que $G=L\left(\mathcal{F}^{\prime}\right)$.

En vista del Teorema 5.14, la respuesta a nuestro problema sólo depende de que $L\left(\mathcal{F}^{\prime}\right)$ sea una solución o no. Dos condiciones deben cumplirse naturalmente para que lo sea, a saber, todos los miembros de $\mathcal{T}$ deben ser árboles clique de $L\left(\mathcal{F}^{\prime}\right)$ y ningún otro árbol puede ser un árbol clique de $L\left(\mathcal{F}^{\prime}\right)$.

Si $L\left(\mathcal{F}^{\prime}\right)$ es solución y decidiéramos aplicarle el procedimiento del Teorema 4.2, entonces debemos conocer a la familia de cliques de $L\left(\mathcal{F}^{\prime}\right)$. Por la Proposición 4.14, la familia de cliques de $L\left(\mathcal{F}^{\prime}\right)$ consiste de todos los conjuntos $D_{v}=\left\{F \in \mathcal{F}^{\prime}: v \in F\right\}, v \in V$.

Si se quiere dar un peso a cada arista del grafo clique, notemos que $\left|D_{u} \cap D_{v}\right|=\mid\{F \in$ $\mathcal{F}:\{u, v\} \subseteq F\} \mid$. Además, si $T$ y $T^{\prime}$ son dos árboles en $\mathcal{T}$ tales que $T^{\prime}=T-w x+u v$, se tiene que $D_{u} \cap D_{v}=D_{w} \cap D_{x}$. Esto motiva el siguiente resultado:

Teorema 5.15. Sean $\mathcal{T}$ una familia de árboles, todos con conjunto de vértices igual a $V, T_{1} \in \mathcal{T}$ y $\mathcal{F}=\left\{\mathcal{T}[u, v]: \quad u v \in E\left(T_{1}\right)\right\}$. Para cada par $u, v \in V, u \neq v$, definamos $D_{u v}=\{F \in$ $\mathcal{F}:\{u, v\} \subseteq F\}$. Entonces, existe un grafo cordal $G$ tal que $\tau(G)=\mathcal{T}$ si y sólo si se satisfacen las siguientes condiciones:

1. Para todo $F \in \mathcal{F}$ y para todo $T \in \mathcal{T}, T[F]$ es un subárbol de $T$.

2. Para todos $u, v \in V, u \neq v, T \in \mathcal{T}$ y $w x \in E(T)$ tal que $\{w, x\} \subseteq T[u, v]$ y $D_{u v}=D_{w x}$, $T-w x+u v \in \mathcal{T}$.

Demostración. Supongamos que existe un grafo cordal $G$ tal que $\tau(G)=\mathcal{T}$. Definamos a $\mathcal{F}^{\prime}$ como se hizo en el Teorema 5.14. Entonces, $\tau\left(L\left(\mathcal{F}^{\prime}\right)\right)=\mathcal{T}$. Como la familia dual de los cliques de $L\left(\mathcal{F}^{\prime}\right)$ es isomorfa a $\mathcal{F}^{\prime}$ (ver la demostración de la Proposición 5.12), se satisface la condición 1 .

Sea $T$ un árbol cualquiera de $\mathcal{T}$ y supongamos que $w$ y $x$ son dos vértices adyacentes en $T$ y contenidos en $T[u, v]$ de modo que $D_{u v}=D_{w x}$. Entonces, por el Teorema 4.2 y el comentario previo a este teorema, $T+u v-w x$ es un árbol clique de $L\left(\mathcal{F}^{\prime}\right)$, es decir, $T+u v-w x \in \mathcal{T}$.

Recíprocamente, supongamos que las condiciones 1. y 2. se cumplen. Entonces, por la condición 1., $L\left(\mathcal{F}^{\prime}\right)$ es un grafo cordal tal que $\mathcal{T} \subseteq \mathcal{T}\left(L\left(\mathcal{F}^{\prime}\right)\right)$. Sean ahora $T \in \mathcal{T}$ y $T^{\prime}$ adyacente a $T$ en $H_{\tau\left(L\left(\mathcal{F}^{\prime}\right)\right)}$. Consideremos las aristas $u v$ y $w x$ tales que $T^{\prime}=T-w x+u v$. Entonces, $D_{u v}=D_{w x}$ y, por la condición 2., $T^{\prime} \in \mathcal{T}$. De este razonamiento y el hecho de que, por la Proposición 5.5, $H_{\tau\left(L\left(\mathcal{F}^{\prime}\right)\right)}$ es conexo, se desprende que $\mathcal{T}=\tau\left(L\left(\mathcal{F}^{\prime}\right)\right)$. 
Es claro que $\mathcal{F}$ y los conjuntos $D_{u v}$ pueden ser encontrados en tiempo polinomial con respecto a $|\mathcal{T}|$ y $|V|$. La condición 1. también puede ser testeada en tiempo polinomial. Además, por cada par de vértices distintos $u$ y $v$ y $T \in \mathcal{T}$, el número de aristas $w x$ en $T$ tales que $D_{u v}=D_{w x}$ no es mayor a $|V|-1$. Por lo tanto, el número de operaciones necesarias para testear a la condición 2. es también polinomial. Por consiguiente, todo el problema puede ser resuelto polinomialmente. También podríamos haber arribado a esta conclusión usando los resultados de la sección anterior.

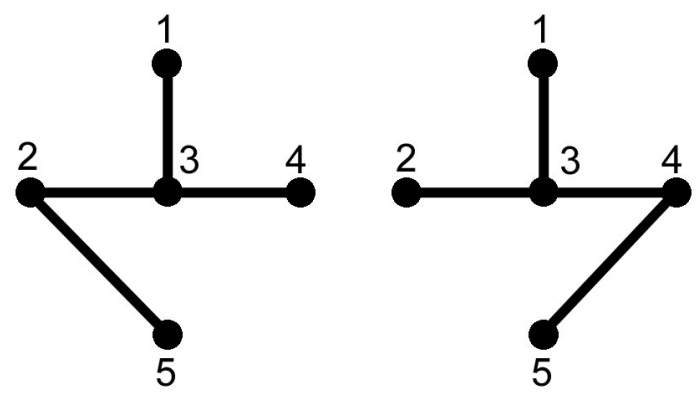

Figura 5.3: Una familia de árboles que satisface la condición 1 del Teorema 5.15, pero que no satisface la condición 2 .

Veamos ahora algunos ejemplos. Consideremos los árboles de la Figura 5.3. Para ellos, $\mathcal{F}=$ $\{\{1,3\},\{2,3\},\{3,4\},\{2,3,4,5\}\}$. Esta familia claramente satisface la condición 1 del Teorema 5.15. Sin embargo, $D_{25}=D_{35}$ y el árbol obtenido al removerle la arista 25 al primer árbol de la Figura 5.3 y agregarle 35 no está en la familia. Por lo tanto, no se trata de una familia de árboles clique de un grafo cordal porque no se satisface la condición 2 .

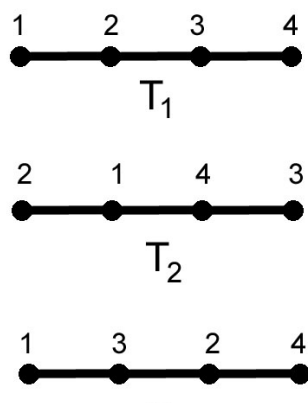

$\mathrm{T}_{3}$
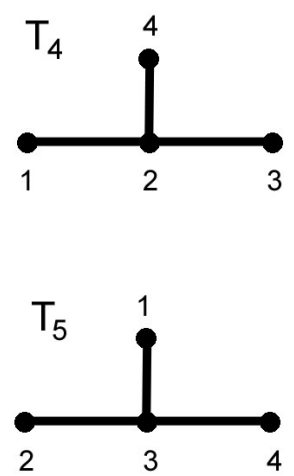

Figura 5.4: Una familia de árboles que satisface la condición 2 del Teorema 5.15 pero que no satisface la condición 1.

En el siguiente ejemplo, en cambio, se va a satisfacer la condición 2, pero no la condición 1. Sea $\mathcal{T}$ la familia de árboles en la Figura 5.4. Entonces, $\mathcal{F}=\{\{1,2,3\},\{2,3,4\},\{1,2,3,4\}\}$. Las únicas igualdades entre conjuntos $D_{u v}$ están dadas por $D_{12}=D_{13}$ y $D_{24}=D_{34}$. El hecho de que $T_{1}-12+13, T_{1}-34+24, T_{3}-13+12, T_{3}-24+34, T_{4}-12+13, T_{4}-24+34, T_{5}-13+12$ y $T_{5}-34+24$ estén todos en $\mathcal{T}$ implica que se satisface la condición 2 . Sin embargo, $T_{2}[\{1,2,3\}]$ y 
$T_{2}[\{2,3,4\}]$ no son subárboles de $T_{2}$. Por lo tanto, no se satisface la condición 1 . Resulta así que $\mathcal{T}$ tampoco es una familia de árboles clique de un grafo cordal.
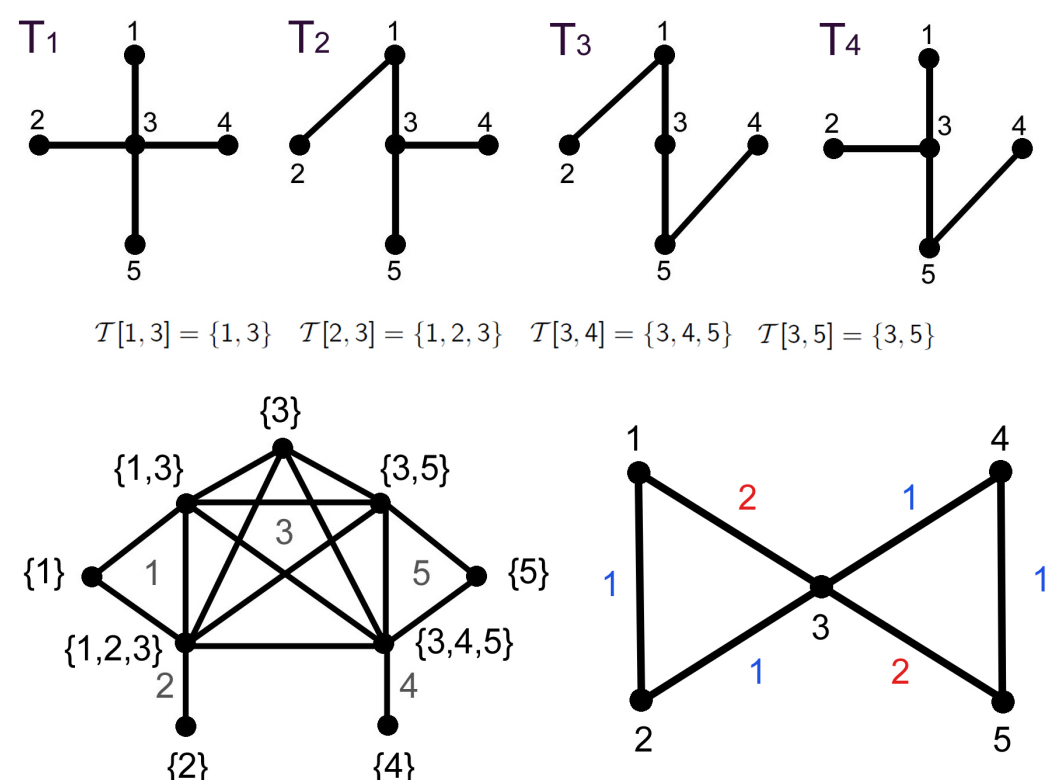

Figura 5.5: Una familia de árboles para la cual la respuesta a nuestro problema es afirmativa y el grafo cordal, dado por el Teorema 5.14, que los tiene a ellos como familia de árboles clique. Se puede ver su grafo clique valuado abajo a la derecha. Todo árbol generador de peso máximo suyo debe tener a las aristas 13 y 35. Para completarlo, debemos elegir entre 12 y 23 y entre 34 y 45. Esto confirma que la familia de árboles clique del grafo es justamente la de arriba.

Finalmente, sea ahora $\mathcal{T}$ la familia de árboles en la Figura 5.5, la cual ya había sido considerada en la Figura 5.2. Sabemos por la sección anterior que ésta es una familia de árboles clique de algún grafo cordal. Se deja al lector comprobar que las condiciones 1 y 2 se satisfacen. $\mathcal{F}=\{\{1,3\},\{3,5\},\{1,2,3\},\{3,4,5\}\}$, y el grafo $L\left(\mathcal{F}^{\prime}\right)$ aparece en la parte inferior de la Figura 5.5. Es simple utilizar el Teorema 4.2 para verificar que $\mathcal{T}=\tau\left(L\left(\mathcal{F}^{\prime}\right)\right)$.

\subsection{Encontrando todos los grafos cordales con familia de árboles clique dada}

Se usa en esta sección todo el conocimiento obtenido en la anterior para encontrar, dada una familia $\mathcal{T}$ de árboles con conjunto de vértices igual a $V$, una fórmula general para todos los grafos cordales que tienen a $\mathcal{T}$ como familia de árboles clique.

En primer lugar, dado un grafo cordal $G$, caracterizamos a todos los grafos cordales con los mismos árboles clique que $G$.

Todo grafo está determinado por la familia dual de sus cliques al ser igual al grafo de intersección de ésta. Para el caso de $G$, los conjuntos $\mathcal{C}_{S}$, siendo $S$ un separador minimal de vértices, pueden ser expresados como intersección de miembros de la familia dual de los cliques. Si $G^{\prime}$ es otro grafo cordal con los mismos árboles clique que $G$, entonces los conjuntos de la forma $\mathcal{C}_{S}, S \in \mathcal{S}\left(G^{\prime}\right)$, son los mismos que los de $G$ porque, como se vio en la Proposición 5.13, existe una expresión para ellos en términos de los árboles clique. Similarmente, estos conjuntos 
también pueden expresarse como la intersección de miembros de la familia dual de cliques de $G^{\prime}$. Estas ideas y otras más conducen al siguiente teorema:

Teorema 5.16. Sean $G$ y $G^{\prime}$ dos grafos cordales. Entonces, $G$ y $G^{\prime}$ tienen los mismos árboles clique si y sólo si $G^{\prime}=L(\mathcal{F})$, siendo $\mathcal{F}$ una subfamilia separadora de $\mathcal{S C}(G)$ tal que, para todo $S \in \mathcal{S}(G), \bigcap_{F \in \mathcal{F}, \mathcal{C}_{S} \subseteq F} F=\mathcal{C}_{S}$.

Demostración. Supongamos que $G^{\prime}=L(\mathcal{F})$, siendo $\mathcal{F}$ una familia como la del enunciado del teorema.

Si repetimos los razonamientos de la parte (b) de la Proposición 5.12, obtenemos que $D \mathcal{C}\left(G^{\prime}\right) \cong$ $\mathcal{F}$. Por lo tanto, como $\mathcal{F} \subseteq \mathcal{S C}(G)$, todo árbol clique de $G$ es un árbol clique de $G^{\prime}$.

Sea ahora $T$ un árbol clique de $G^{\prime}$. Entonces, todo miembro de $\mathcal{F}$ induce un subárbol de $T$. La condición de que, para todo $S \in \mathcal{S}(G), \bigcap_{F \in \mathcal{F}, \mathcal{C}_{S} \subseteq F} F=\mathcal{C}_{S}$, implica que $\mathcal{C}_{S}$ induce un subárbol de $T$. Luego, por la Proposición 5.12 y el Teorema 4.13, $T$ es un árbol clique de $G$. Se sigue que $G$ y $G^{\prime}$ tienen los mismos árboles clique.

Recíprocamente, supongamos que $G$ y $G^{\prime}$ son dos grafos cordales con los mismos árboles clique. Entonces, $\mathcal{S C}(G)=\mathcal{S C}\left(G^{\prime}\right)$. Sea $\mathcal{F}=D \mathcal{C}\left(G^{\prime}\right)$. Luego, $G^{\prime} \cong L(\mathcal{F})$ y, por la afirmación previa, $\mathcal{F} \subseteq \mathcal{S C}(G)$. Sean ahora $S \in \mathcal{S}(G)$ y $C_{1} C_{2} \in E(\tau(G))$ tales que $C_{1} \cap C_{2}=S$. La igualdad en los árboles clique para ambos grafos implica que $\mathcal{T}_{G}\left[C_{1}, C_{2}\right]=\mathcal{T}_{G^{\prime}}\left[C_{1}, C_{2}\right]$. Por la Proposición 5.13, $\mathcal{T}_{G}\left[C_{1}, C_{2}\right]=\mathcal{C}_{S}$. Por otro lado, también por la Proposición 5.13, $\mathcal{T}_{G^{\prime}}\left[C_{1}, C_{2}\right]$ puede ser expresado como la intersección de miembros de la familia dual de cliques de $G^{\prime}$, es decir, como la intersección de miembros de $\mathcal{F}$. Se sigue inmediatamente que $\bigcap_{F \in \mathcal{F}, \mathcal{C}_{S} \subseteq F} F=\mathcal{C}_{S}$.

Sabemos que, dada $\mathcal{T}$, cuando $\mathcal{T}$ es afirmativamente la familia de árboles clique de algún grafo cordal, podemos usar el Teorema 5.14 para construir un grafo cordal con las condiciones requeridas. Si a esto se la suma el Teorema 5.16, seremos capaces de caracterizar a todos los grafos cordales que tienen a $\mathcal{T}$ como familia de árboles clique.

Sean $\mathcal{T}, T_{1}, \mathcal{F}$ y $\mathcal{F}^{\prime}$ los mismos que en el Teorema 5.14, o sea, $\mathcal{T}$ una familia de árboles con conjunto $V$ de vértices, $T_{1} \in \mathcal{T}, \mathcal{F}=\left\{\mathcal{T}[u, v]: u v \in E\left(T_{1}\right)\right\}$ y $\mathcal{F}^{\prime}=\mathcal{F} \cup\{\{v\}: v \in V\}$. Definamos a $S p(\mathcal{F})$ como la familia constituida por los conjuntos unitarios contenidos en $V$ y por todos los conjuntos que pueden expresarse como uniones conexas de miembros de $\mathcal{F}$. Entonces se tiene:

Teorema 5.17. Sea $C h(\mathcal{T})=\{G: \tau(G)=\mathcal{T}\}$. Entonces, se tiene la siguiente alternativa:

$* C h(\mathcal{T})=\emptyset$.

* $G \in C h(\mathcal{T})$ si y sólo si $G=L\left(\mathcal{F}^{\prime \prime}\right)$, siendo $\mathcal{F}^{\prime \prime}$ una subfamilia separadora de $S p(\mathcal{F})$ tal que, para toda $u v \in E\left(T_{1}\right), \bigcap_{F \in \mathcal{F}^{\prime \prime},\{u, v\} \subseteq F} F=\mathcal{T}[u, v]$.

Demostración. Supongamos que $C h(\mathcal{T}) \neq \emptyset$. Entonces, por el Teorema 5.14, $L\left(\mathcal{F}^{\prime}\right) \in C h(\mathcal{T})$ y, por la Proposición 5.13 y el Teorema $4.13, S p(\mathcal{F})=\mathcal{S C}\left(L\left(\mathcal{F}^{\prime}\right)\right)$. Sean $u v \in E\left(T_{1}\right)$ y $F \in S p(\mathcal{F})$ tales que $\{u, v\} \subseteq F$. Por lo visto arriba, $F$ induce un subárbol de todo $T \in \mathcal{T}$. Entonces, $T[u, v] \subseteq F$ para todo $T \in \mathcal{T}$ y, por ende, $\mathcal{T}[u, v] \subseteq F$. Luego, para todo $F \in S p(\mathcal{F}),\{u, v\} \subseteq F$ si y sólo si $\mathcal{T}[u, v] \subseteq F$.

La conclusión se obtiene de aplicar el Teorema 5.16 a $L\left(\mathcal{F}^{\prime}\right)$. 
Como ejemplo, consideremos la familia $\mathcal{T}$ en la Figura 5.6. Se cumple que $C h(\mathcal{T}) \neq \emptyset$, $\mathcal{F}=\{\{1,3\},\{2,3\},\{3,4\},\{2,3,4,5\}\}$, y $L\left(\mathcal{F}^{\prime}\right)$ es igual al grafo $G$ en la figura. La figura muestra también a otro grafo $G^{\prime} \in C h(\mathcal{T})$. $G^{\prime}$ puede ser visto como el grafo de intersección de la familia $\mathcal{F}^{\prime \prime}=\{\{1\},\{2\},\{4\},\{5\},\{1,2,3\},\{1,3,4\},\{2,3,4,5\}\}$. No es difícil ver que $\mathcal{F}^{\prime \prime} \subseteq S p(\mathcal{F})$.

Sea $T_{1}$ el árbol arriba a la izquierda en la figura.

Los miembros de $\mathcal{F}^{\prime \prime}$ que contienen a $\{1,3\}$ son $\{1,2,3\}$ y $\{1,3,4\}$. La intersección de ellos es igual a $\mathcal{T}[1,3]$.

Los miembros de $\mathcal{F}^{\prime \prime}$ que contienen a $\{2,3\}$ son $\{1,2,3\}$ y $\{2,3,4,5\}$. La intersección de ellos es igual a $\mathcal{T}[2,3]$.

Los miembros de $\mathcal{F}^{\prime \prime}$ que contienen a $\{3,4\}$ son $\{1,3,4\}$ y $\{2,3,4,5\}$. La intersección de ellos es igual a $\mathcal{T}[3,4]$.

El único miembro de $\mathcal{F}^{\prime \prime}$ que contiene a $\{2,5\}$ es $\{2,3,4,5\}$, que es igual a $\mathcal{T}[2,5]$.

Por lo tanto, vemos que este ejemplo concuerda con el contenido del Teorema 5.17.
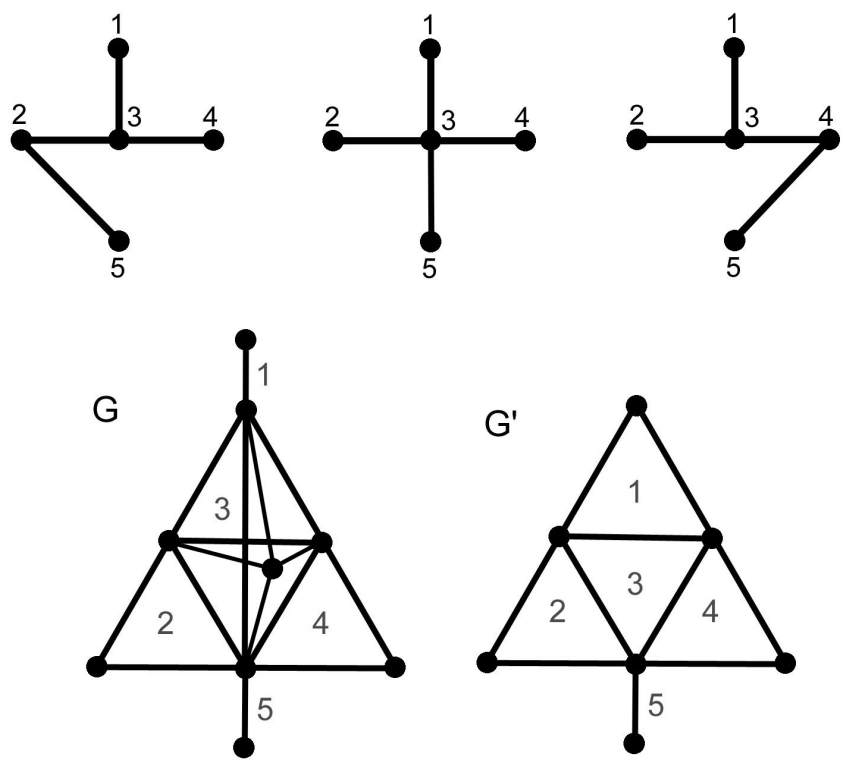

Figura 5.6: Una familia de árboles y dos grafos cordales que los tienen como árboles clique.

\subsection{Detectando familias de árboles compatibles de los grafos dualmente cordales}

El tema de esta sección no es muy diferente al de las anteriores. Pero nos enfocaremos ahora en los grafos dualmente cordales. Más precisamente, dada una familia $\mathcal{T}$ de árboles, todos con conjunto $V$ de vértices, se debe determinar si existe un grafo dualmente cordal cuya familia de árboles compatibles es $\mathcal{T}$. No profundizaremos tanto en los detalles en esta sección, pues está más bien pensada como una introducción a la cuestión.

Es interesante notar que una familia de árboles compatibles de un grafo dualmente cordal es también una familia de árboles clique de un grafo cordal. Más aun, se puede decir lo siguiente: 
Proposición 5.18. Sea $\mathcal{T}$ una familia de árboles con conjunto de vértices igual a $V$. Entonces, $\mathcal{T}$ es la familia de árboles compatibles de un grafo dualmente cordal si y sólo si $\mathcal{T}$ es la familia de árboles clique de un grafo básicamente cordal.

Demostración. Usar la definición de grafo básicamente cordal y el Teorema 4.15.

Sin embargo, no toda familia de árboles clique de un grafo cordal es la familia de árboles compatibles de algún grafo dualmente cordal. Consideremos los árboles de la Figura 5.6. Éstos son los árboles clique de los grafos cordales que están abajo de ellos. Veamos que no hay ningún grafo dualmente cordal que los tenga como familia de árboles compatibles. Si tal grafo existiera, entonces 13 sería una de sus aristas y, por la definición de árbol compatible, $\{2,3,4,5\}$ sería un conjunto completo. Pero el grafo debería tener más aristas pues, de lo contrario, el camino 13245 sería un árbol compatible. Supongamos que 12 es otra de sus aristas. Entonces, 2 es un vértice universal y así la estrella centrada en 2 se convierte en un árbol compatible, lo cual es absurdo. La misma contradicción surgirá si se agrega cualquier otra arista. Luego, ningún grafo dualmente cordal tiene a los árboles de la Figura 5.6 como su familia de árboles compatibles.

Como consecuencia de la Proposición 5.18, podemos restringir el análisis del problema de esta sección a familias de árboles clique de grafos cordales.

Sean $\mathcal{T}$ la familia de árboles clique de un grafo cordal y $T \in \mathcal{T}$. Sea $\mathcal{F}=\{\mathcal{T}[a, b]: a, b \in$ $V(T)\}$. No es difícil probar que $\mathcal{F}$ es una familia de subárboles de todo árbol en $\mathcal{T}$.

Si existe un grafo dualmente cordal $G$ tal que la familia de árboles compatibles de $G$ es $\mathcal{T}$, consideremos a $\mathcal{F}^{\prime}=\{\mathcal{T}[a, b]: a b \in E(G)\}$. Usando las propiedades de los árboles compatibles, inferimos que los miembros de $\mathcal{F}^{\prime}$ son conjuntos completos de $G$. Por consiguiente, $G=S\left(\mathcal{F}^{\prime}\right)$. A su vez, una técnica similar a la de la demostración de la Proposición 5.13 nos permite probar que, si $u, v$ son adyacentes en $T$, entonces $\mathcal{T}[u, v]=\bigcap_{w \in N[u] \cap N[v]} N[w]=\{w \in V(G): N[u] \cap N[v] \subseteq$ $N[w]\}$.

Recíprocamente, supongamos que existe $\mathcal{F}^{\prime} \subseteq \mathcal{F}$ tal que $S\left(\mathcal{F}^{\prime}\right)=G$ y, para toda $u v \in E(T)$, $\bigcap_{w \in N_{G}[u] \cap N_{G}[v]} N_{G}[w]=\mathcal{T}[u, v]$. Por la construcción de $G, T$ es compatible con él. Sea $G^{\prime}$ un grafo cordal cuya familia de árboles clique es $\mathcal{T}$. La igualdad en este párrafo, combinada con las Proposiciones 4.19 y 5.13 y los Teoremas 4.13 y 4.18 , implica que la base de $\mathcal{S D C}(G)$ es igual a la base de $\mathcal{S C}\left(G^{\prime}\right)$. Luego, por el Teorema 4.11, los árboles clique de $G^{\prime}$ son exactamente los árboles compatibles de $G$, es decir, la familia de árboles compatibles de $G$ es $\mathcal{T}$.

Hemos probado entonces el siguiente teorema:

Teorema 5.19. Sean $\mathcal{T}$ una familia de árboles clique de un grafo cordal, $T \in \mathcal{T}$ y $\mathcal{F}=$ $\{\mathcal{T}[a, b]: a, b \in V(T)\}$. Entonces, $\mathcal{T}$ es la familia de árboles compatibles de un grafo dualmente cordal $G$ si y sólo si $G=S\left(\mathcal{F}^{\prime}\right)$, siendo $\mathcal{F}^{\prime}$ una subfamilia de $\mathcal{F}$, y, para toda uv $\in E(T)$, el conjunto de vértices $w$ tales que $N_{G}[u] \cap N_{G}[v] \subseteq N_{G}[w]$ es igual a $\mathcal{T}[u, v]$.

El Teorema 5.19 transforma a nuestro problema en uno de teoría de conjuntos. Veamos un par de ejemplos.

Consideremos los árboles de la Figura 5.5. Son los árboles clique del grafo cordal que también ahí se muestra. La base asociada a este grafo cordal está compuesta por $\{1,2,3\},\{1,3\},\{3,4,5\}$ y $\{3,5\}$. Sin embargo, no es difícil ver que la dos sección de estos conjuntos es un grafo dualmente cordal con más de cuatro árboles compatibles. Pero si tomamos a $\mathcal{T}[1,2]=\{1,2,3\}, \mathcal{T}[1,5]=$ $\{1,3,5\}$ y $\mathcal{T}[3,4]=\{3,4,5\}$, obtenemos así una familia en las condiciones del Teorema 5.19. Luego, la familia de árboles de la Figura 5.5 es la familia de árboles compatibles de un grafo dualmente cordal (ver Figura 5.7). 


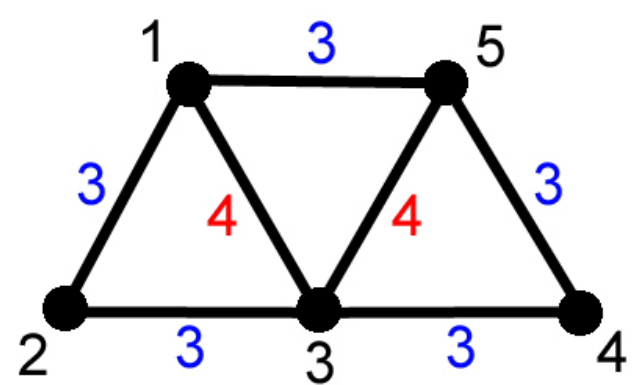

Figura 5.7: Un grafo dualmente cordal y una valuación para sus aristas, en concordancia con el Teorema 4.3, que nos permite ver que sus árboles compatibles son los de la Figura 5.5.

Consideremos nuevamente los árboles de la Figura 5.6 y sea $T$ el árbol a la izquierda. Entonces, $\mathcal{T}[1,2]=\{1,2,3\}, \mathcal{T}[1,3]=\{1,3\}, \mathcal{T}[1,4]=\{1,3,4\} \mathcal{T}[1,5]=\{1,2,3,4,5\}, \mathcal{T}[2,3]=$ $\{2,3\}, \mathcal{T}[2,4]=\{2,3,4\}, \mathcal{T}[2,5]=\{2,3,4,5\}, \mathcal{T}[3,4]=\{3,4\}$ y $\mathcal{T}[3,5]=\{2,3,4,5\}$.

Supongamos que existe una familia $\mathcal{F}^{\prime}$ que satisface las condiciones del Teorema 5.19. Es evidente que $\mathcal{T}[1,5]$ no debe estar en $\mathcal{F}^{\prime}$. Además, $\mathcal{T}[2,5]$ debe estar en $\mathcal{F}^{\prime}$, pues de lo contrario $N_{S\left(\mathcal{F}^{\prime}\right)}[2] \cap N_{S\left(\mathcal{F}^{\prime}\right)}[5]=\emptyset$. Como $\mathcal{T}[2,3]=\{2,3\}$, necesitamos que $\mathcal{T}[1,2] \in \mathcal{F}^{\prime}$ y que $\mathcal{T}[1,4] \notin \mathcal{F}^{\prime}$ para que $N_{S\left(\mathcal{F}^{\prime}\right)}[2] \cap N_{S\left(\mathcal{F}^{\prime}\right)}[3] \nsubseteq N_{S\left(\mathcal{F}^{\prime}\right)}[4]$. Sin embargo, si tenemos en cuenta que $\mathcal{T}[3,4]=\{3,4\}$, la conclusión que se obtendrá esta vez es que $\mathcal{T}[1,4] \in \mathcal{F}^{\prime}$ y que $\mathcal{T}[1,2] \notin \mathcal{F}^{\prime}$, lo cual nos da una contradicción.

Por lo tanto, hemos visto nuevamente que la familia de árboles de la Figura 5.6 no es la familia de árboles compatibles de ningún grafo dualmente cordal.

Se pudo aplicar al Teorema 5.19 de manera satisfactoria en los ejemplos porque las familias consideradas eran pequeñas. Sin embargo, se podría complicar mucho en instancias más generales. Se deja como problema abierto hallar la complejidad de determinar si una familia de árboles es la familia de árboles compatibles de algún grafo dualmente cordal. 


\section{Capítulo 6}

\section{Conclusiones}

Puede considerarse que los principales aportes de este trabajo son:

- Mostrar cómo, a pesar de sus diferentes características, los vértices simpliciales, con máximo vecino, p-simpliciales, doblemente simpliciales y simples conducen a propiedades análogas de los grafos cordales, dualmente cordales, cordal-potentes, doblemente cordales y fuertemente cordales.

- Lograr un mayor entendimiento de las caracterizaciones de los grafos dualmente cordales a través de un estudio detallado de los árboles compatibles.

- Introducir a los grafos básicamente cordales y encontrar una caracterización de ellos que permite identificarlos con suficiente facilidad.

- Mostrar, dado un grafo dualmente cordal $G$, cómo encontrar los grafos básicamente cordales cuyos árboles clique son los árboles compatibles de $G$. De esto se desprende que todo problema acerca de los árboles compatibles de un grafo dualmente cordal se reduce a un problema acerca de los árboles clique de un grafo cordal, lo cual es de relevancia porque varios problemas acerca de los árboles clique de un grafo cordal, como el del leafage, ya han sido estudiados.

- Mostrar cómo se puede resolver eficientemente en tiempo polinomial un par de problemas vinculados a los árboles: determinar si un conjunto de vértices constituye el conjunto de hojas de algún árbol compatible de un grafo dualmente cordal dado; y, dada una familia de árboles, decidir si es la familia de árboles clique de algún grafo cordal.

De continuar pensando en todas estas cuestiones, aparecen con facilidad más problemas que quedan abiertos. Como ejemplo, se mencionarán aquí tres de ellos:

- Dado un grafo dualmente cordal $G$ y una familia $\mathcal{F}$ cuyos elementos son los vértices de $G$, ¿Cuáles son las condiciones necesarias y suficientes que $\mathcal{F}$ debe satisfacer para que valga que, para todo $T$ árbol generador, $T$ es compatible con $G$ si y sólo si todo miembro de $\mathcal{F}$ induce un subárbol de $T$ ?

- Dos subclases de los grafos cordales, los grafos $D V$ y $R D V$, pueden ser caracterizadas por tipos especiales de árboles clique y poseen clases duales que a su vez pueden ser caracterizadas por tipos especiales de árboles compatibles. Por lo tanto, los grafos básicamente $D V / R D V$ pueden ser definidos similarmente a cómo fueron definidos los grafos básicamente cordales. ¿Qué propiedades tienen estas clases y cómo pueden ser caracterizadas? 
- Finalmente, como se indica al final del Capítulo 5, es de interés en este contexto conocer la complejidad algorítmica de determinar, dada una familia $\mathcal{T}$ de árboles, si $\mathcal{T}$ es la familia de árboles compatibles de algún grafo dualmente cordal.

Éstas y algunas más son preguntas que el autor se plantea para intentar resolver un un corto o mediano plazo. 


\section{Bibliografía}

[1] H. J. Bandelt y E. Prisner, Clique graphs and Helly graphs, Journal of Combinatorial Theory B 51 (1991), 34-45.

[2] C. Berge, Hypergraphs, North-Holland, Amsterdam, 1989.

[3] A. Brandstädt, F. Dragan, V. Chepoi y V. Voloshin, Dually chordal graphs, SIAM J. Discrete Math. 11 (1998), 437-455.

[4] G.A. Dirac, On rigid circuit graphs, Abh. Math. Sem. Univ. Hamburg 25 (1961), 71-76.

[5] F. Dragan, HT-graphs: centers, connected r-domination and Steiner trees, Comput. Sci. J. Moldova 1 (1993), 64-83.

[6] F. Dragan, C. Prisacaru y V. Chepoi, Location problems in graphs and the Helly property (1987) (en ruso, versión parcial en Diskretnaja Matematika 4 (1992), 67-73).

[7] P. Duchet, Classical Perfect Graphs, An introduction with emphasis on triangulated and interval graphs, Ann. Discrete Math. 21 (1984), 67-96.

[8] M. Farber, Characterizations of strongly chordal graphs, Discrete Math. 43 (1983), 173-189.

[9] M. Farber y R.E. Jamison, Convexity in graphs and hypergraphs, SIAM Journal on Algebraic and Discrete Methods 7 (1986), 433-444.

[10] D.R. Fulkerson y O.A. Gross, Incidence matrices and interval graphs, Pacific J. Math. 15 (1965), 835-855.

[11] F. Gavril, The intersection graphs of subtrees in trees are exactly the chordal graphs, J. Combin. Theory Ser. B 116 (1974), 47-56.

[12] M.C. Golumbic, Algorithmic Graph Theory and Perfect Graphs, Second edition, Annals of Discrete Mathematics 57, Elsevier, 2004.

[13] M. Gutierrez, Tree-Clique Graphs, Estudos e comunicações do Instituto de Matemática, Universidade Federal do Rio de Janeiro 63 (1996), 7-26.

[14] M. Gutierrez y J. Meidanis, Algebraic theory for the clique operator, J. Braz. Comp. Soc. 7 (2001), 53-64.

[15] M. Habib y J. Stacho, Polynomial-time Algorithm for the Leafage of Chordal Graphs, Algorithms - ESA 2009, Lecture Notes in Comp. Sci. 5757 (2009), pp. 290-300.

[16] M. Habib y J. Stacho, Reduced clique graphs of chordal graphs, European Journal of Combinatorics 33 (2012), 712-735. 
[17] J.B. Kruskal, On the shortest spanning tree of a graph and the traveling salesman problem, Proceedings of the American Mathematical Society 7 (1956), 48-50.

[18] T.A. McKee, How chordal graphs work, Bulletin of the ICA 9 (1993), 27-39.

[19] F.R. McMorris, T.J. Warnow y T. Wimer, Triangulating vertex-colored graphs, SIAM J. Discrete Math. 7 (1994), 296-306.

[20] J. Meidanis y J. Setubal, Introduction to Computational Molecular Biology, PWS Publishing, Boston MA, 1997.

[21] C.L. Monma y V.K. Wei, Intersection Graphs of Paths in a Tree, Journal of Combinatorial Theory B 41 (1986), 141-181.

[22] J.W. Moon, Various proofs of Cayley's formula for counting trees, en: F. Harary (Ed.), A Seminar on Graph Theory, Holt, Rinehart and Winston, NewYork, 1967, 70-78.

[23] P.J. Slater, A characterization of SOFT hypergraphs, Canad. Math. Bull. 21 (1978), 335337.

[24] P. Sreenivasa Kumara y C.E. Veni Madhavanb, Clique tree generalization and new subclasses of chordal graphs, Discrete Applied Mathematics 117 (2002), 109-131.

[25] J. L. Szwarcfiter y C.F. Bornstein, Clique graphs of chordal and path graphs, SIAM Journal on Discrete Mathematics 86 (1990), 331-336. 